\title{
Herschel-HOBYS study of the earliest phases of high-mass star formation in NGC $6357^{\star, \star \star}$
}

\author{
D. Russeil ${ }^{1}$, M. Figueira ${ }^{1,2}$, A. Zavagno ${ }^{1}$, F. Motte ${ }^{3}$, N. Schneider ${ }^{4,5}$, A. Men'shchikov ${ }^{6}$, S. Bontemps ${ }^{5}$, \\ P. André ${ }^{6}$, L. D. Anderson ${ }^{7,8,9}$, M. Benedettini ${ }^{10}$, P. Didelon ${ }^{6}$, J. Di Francesco ${ }^{10}$, D. Elia ${ }^{10}$, \\ V. Könyves ${ }^{6,11}$, Q. Nguyen Luong ${ }^{12,13}$, T. Nony ${ }^{3}$, S. Pezzuto ${ }^{10}$, K. L. J. Rygl ${ }^{14}$, E. Schisano ${ }^{10}$, \\ L. Spinoglio ${ }^{10}$, J. Tigé ${ }^{1}$, and G. J. White ${ }^{15,16}$
}

\author{
${ }^{1}$ CNRS, CNES, LAM, Aix-Marseille Univ., Marseille, France \\ e-mail: delphine.russeil@lam.fr \\ 2 National Centre for Nuclear Research, ul. Hoza 69, 00-681 Warszawa, Poland \\ ${ }^{3}$ CNRS, IPAG, Université Grenoble Alpes, 38000 Grenoble, France \\ ${ }^{4}$ I. Physik. Institut, University of Cologne, 50937 Cologne, Germany \\ ${ }^{5}$ Laboratoire d'Astrophysique de Bordeaux, CNRS/INSU, Université de Bordeaux, UMR 5804, France \\ ${ }^{6}$ Laboratoire AIM, CEA/IRFU CNRS/INSU Université Paris Diderot, CEA-Saclay, 91191 Gif-sur-Yvette Cedex, France \\ ${ }^{7}$ Department of Physics and Astronomy, West Virginia University, Morgantown WV 26506, USA \\ 8 Adjunct Astronomer at the Green Bank Observatory, PO Box 2, Green Bank WV 24944, USA \\ ${ }_{9}^{9}$ Center for Gravitational Waves and Cosmology, West Virginia University, Chestnut Ridge Research Building, Morgantown, \\ WV 26505, USA \\ ${ }^{10}$ INAF - IAPS, Via Fosso del Cavaliere 100, 00133 Rome, Italy \\ 11 Jeremiah Horrocks Institute, University of Central Lancashire, Preston PR1 2HE, UK \\ 12 Korea Astronomy and Space Science Institute, 776 Daedeok daero, Yuseoung, Daejeon 34055, Korea \\ ${ }^{13}$ NAOJ Chile Observatory National Astronomical Observatory of Japan, 2-21-1 Osawa, Mitaka, Tokyo 181-8588, Japan \\ ${ }_{14}$ INAF - Istituto di Radioastronomia \& Italian ALMA Regional Centre, via P. Gobetti 101, 40129 Bologna, Italy \\ ${ }^{15}$ School for Physical Sciences, The Open University, Walton Hall, Milton Keynes, MK7 6AA, UK \\ ${ }^{16}$ RAL Space, STFC Rutherford Appleton Laboratory, Chilton, Didcot, Oxfordshire, OX11 0QX, UK
}

Received 16 July 2018 / Accepted 2 April 2019

\begin{abstract}
Aims. To constrain models of high-mass star formation it is important to identify the massive dense cores (MDCs) that are able to form high-mass star(s). This is one of the purposes of the Herschel/HOBYS key programme. Here, we carry out the census and characterise of the properties of the MDCs population of the NGC $6357 \mathrm{H}$ II region.

Methods. Our study is based on the Herschel/PACS and SPIRE 70-500 $\mu$ m images of NGC 6357 complemented with (sub-)millimetre and mid-infrared data. We followed the procedure established by the Herschel/HOBYS consortium to extract $\sim 0.1 \mathrm{pc}$ massive dense cores using the getsources software. We estimated their physical parameters (temperatures, masses, luminosities) from spectral energy distribution (SED) fitting.

Results. We obtain a complete census of 23 massive dense cores, amongst which one is found to be IR-quiet and twelve are starless, representing very early stages of the star-formation process. Focussing on the starless MDCs, we have considered their evolutionary status, and suggest that only five of them are likely to form a high-mass star.

Conclusions. We find that, contrarily to the case in NGC 6334, the NGC 6357 region does not exhibit any ridge or hub features that are believed to be crucial to the massive star formation process. This study adds support for an empirical model in which massive dense cores and protostars simultaneously accrete mass from the surrounding filaments. In addition, the massive star formation in NGC 6357 seems to have stopped and the hottest stars in Pismis 24 have disrupted the filaments.
\end{abstract}

Key words. stars: massive - stars: formation

\section{Introduction}

High-mass stars $\left(\mathrm{O}-\mathrm{B} 3\right.$ type, $\left.>8 M_{\odot}\right)$ are the ionising sources of $\mathrm{H}$ II regions. They impact the interstellar medium mainly via their UV radiation, the dynamical expansion of their $\mathrm{H}$ II region

* Full Table C1, Tables C2-C5, the reduced Herschel FITS images and the column density FITS image are only available at the CDS via anonymous ftp to cdsarc.u-strasbg. fr (130.79.128.5) or via http:// cdsarc.u-strasbg.fr/viz-bin/qcat?J/A+A/625/A134

${ }^{\star \star}$ Herschel is an ESA space observatory with science instruments provided by European-led Principal Investigator consortia and with important participation from NASA. and supernova events. While their stellar and supernova phases are optically bright, their earliest phase of evolution occurs in cold massive dense cores (MDCs with sizes and volume densities of $\sim 0.1 \mathrm{pc}$ and $>10^{5} \mathrm{~cm}^{-3}$, respectively, see Motte et al. 2007), observable only in the far infra-red (FIR) and submillimetre (sub-mm) domains. Thanks to the development of FIR and sub-mm instrumentation, our understanding of highmass star formation processes become clearer, suggesting a much more dynamical process than the formation of low-mass stars such as accretion streams, associated with converging flows, and cloud hierarchical global collapse (Schneider et al. 2010; Csengeri et al. 2011; Peretto et al. 2014). In particular, 


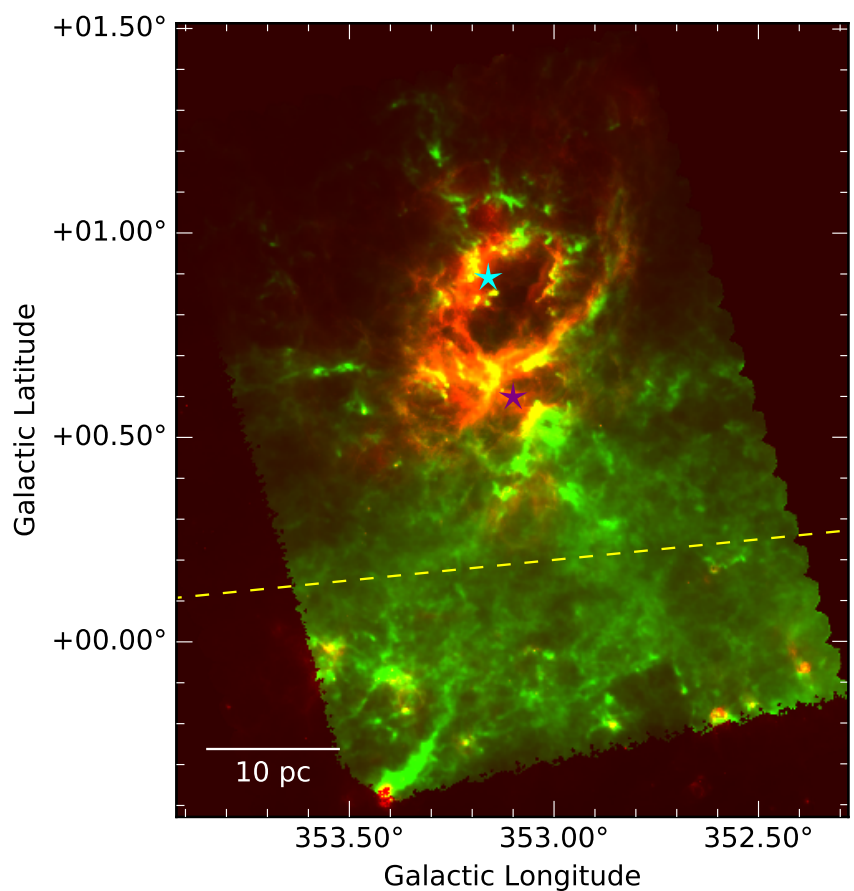

Fig. 1. Herschel-colour image of NGC 6357: $70 \mu \mathrm{m}$ (red, resolution 5.9") and high-resolution column density (green, resolution 18.2"). The coordinates are Galactic coordinates. The dashed yellow line outlines the Galactic latitude above which we assume the emission belongs to NGC 6357 and that we used to build the HOBYS catalogue. The cyan and magenta symbols indicate the position of the OB clusters Pismis 24 and AH03J1725-34.4, respectively.

Tigé et al. (2017), studying massive dense cores (MDCs) in the star-forming region NGC 6334, favour a scenario wherein ridges/hubs, MDCs and high-mass protostellar embryos form and grow simultaneously (see the review of Motte et al. 2018a). A few high-resolution studies have already been performed with (sub-)millimetre interferometers revealing that starless highmass cores are very difficult to find (e.g. Duarte-Cabral et al. 2013; Tan et al. 2013; Nony et al. 2018). Even in NGC 6334 no high-mass pre-stellar cores (corresponding to the high-mass analogues of low-mass pre-stellar cores) were found (Louvet et al. 2019) supporting this.

NGC 6334 has similar velocity and distance to NGC 6357 (the adopted distance is $1.75 \mathrm{kpc}$, Russeil et al. 2012) and since the extinction and the $1.2 \mathrm{~mm}$ emission morphology tend to indicate that they are connected by a filamentary structure (Russeil et al. 2010) we usually consider them as a "twin massive starforming complex". However, contrary to NGC 6357, NGC 6334 exhibits a dense molecular ridge and two hubs (Matthews et al. 2008; Tigé et al. 2017), meaning that it could have a different star-formation history. The main characteristic of NGC 6357 $\left(l=353.4^{\circ}, b=+0.6^{\circ}\right)$ is its shell like morphology identified in $\mathrm{H} \alpha$, and by its surrounding photo-dissociation region (PDR, as seen by its PAH- $8 \mu \mathrm{m}$ emission). Filled by hot gas (Cappa et al. 2011) the cavity is shaped by the ionising open cluster Pismis 24. A shell of fragmented molecular gas was also identified in CO emission (e.g. Massi et al. 1997) and Giannetti et al. (2012) show the presence of a velocity gradient in the region suggesting that the expansion of the ionised gas is pushing the molecular gas. In addition, at least four other H II regions (see Fig. 1 in Russeil et al. 2016), adjacent to the cavity, belong to NGC 6357, one of them being powered by the cluster AH03J1725-34.4 (Dias et al. 2002).
In this paper, we have focussed our study on NGC 6357 based on data (Herschel-HOBYS ${ }^{1}$ imaging survey) and an approach and method similar to that presented by Tigé et al. (2017) for the region NGC 6334. Our main goal is to identify and characterise MDCs in order to compare the massive star-formation processes in both regions. Clouds are hierarchical multi-scale structures, which are sub-divided into $1 \mathrm{pc}$ clumps, $\sim 0.1 \mathrm{pc} \mathrm{MDC}$, and $\sim 0.01 \mathrm{pc}$ cores. Given the Herschel angular resolution $\left(12^{\prime \prime}\right.$ at $160 \mu \mathrm{m})$ and the homogenous $1-3 \mathrm{kpc}$ distances of HOBYS clouds, the HOBYS key programme is dedicated to identify and characterise $0.1 \mathrm{pc}$ MDCs. Since density is a better criterion than mass to evaluate whether a cloud structure has the ability to form high-mass stars we chose to focus on the densest cloud structures. In this paper, we will then define MDCs as massive $\sim 0.03$ to $\sim 0.3$ pc cloud structures whose mass is defined in Sect. 4.1 .

Combining the Herschel-HOBYS data with complementary images described in Sect. 2, we extract the dense cores and characterise their properties (Sect. 3). Section 4 presents a complete sample of MDCs with robust mass estimates. Finally, in Sect. 5, we discuss the MDCs properties in comparison with the ones belonging to NGC 6334. Conclusions are given in Sect. 6 .

\section{Observations}

\subsection{Herschel observations, data reduction, and column density images}

NGC 6357 has been observed by the Herschel space observatory with the PACS (Poglitsch et al. 2010) and SPIRE (Griffin et al. 2010) instruments ${ }^{2}$ as part of the HOBYS (Motte et al. 2010) Key Programme (OBSIDs: 1342204847 and 1342204848). Data were taken in five bands: 70 and $160 \mu \mathrm{m}$ for PACS and 250, 350, and $500 \mu \mathrm{m}$ for SPIRE with FWHM resolutions of 5.9", 11.7", $18.2^{\prime \prime}, 24.9^{\prime \prime}$, and 36.3", respectively. Observations were performed in parallel mode, using both instruments simultaneously, with a scanning speed of $20^{\prime \prime} \mathrm{s}^{-1}$. The size of the observed field is $1.7^{\circ} \times 1.1^{\circ}$, which corresponds to $52 \mathrm{pc} \times 34 \mathrm{pc}$ at a distance of $1.75 \mathrm{kpc}$.

Data were reduced using the Herschel Interactive Processing Environment (HIPE, Ott 2010) ${ }^{3}$ software, version 10.0.2751. Versions 7.0 onwards contain a module which significantly removes the stripping effects that have been observed in SPIRE maps produced with previous HIPE versions. SPIRE nominal and orthogonal maps were separately processed and subsequently combined and reduced for de-stripping, relative gains, and colour correction with HIPE. PACS maps were reduced with HIPE up to Level 1 and, from there up to their final version (Level 3) using Scanamorphos v21.0 (Roussel 2013).

Column density maps were built both at the $36.3^{\prime \prime}$ and $18.2^{\prime \prime}$ resolutions of SPIRE 500 and $250 \mu \mathrm{m}$ data. The procedure used to construct the $36.3^{\prime \prime}$ resolution image uses the SED (Spectral Energy Distribution) fitting method fully described in Hill et al. (2011, 2012). To build the high-resolution column density map a multi-scale decomposition of the imaging data was performed and described in detail in Appendix A of Palmeirim et al. (2013).

\footnotetext{
1 http://hobys-herschel. cea.fr

2 Instrument parameters and calibration are given in the PACS and SPIRE observers manuals. See http://Herschel.esac.esa.int/ Docs/PACS/html/pacs_om.html for PACS and http://Herschel. esac.esa.int/Docs/SPIRE/html/spire_handbook.html for SPIRE.

HIPE has been jointly developed by the Herschel Science Ground Segment Consortium, consisting of ESA, the NASA Herschel Science Center, and the HIFI, PACS, and SPIRE consortia.
} 

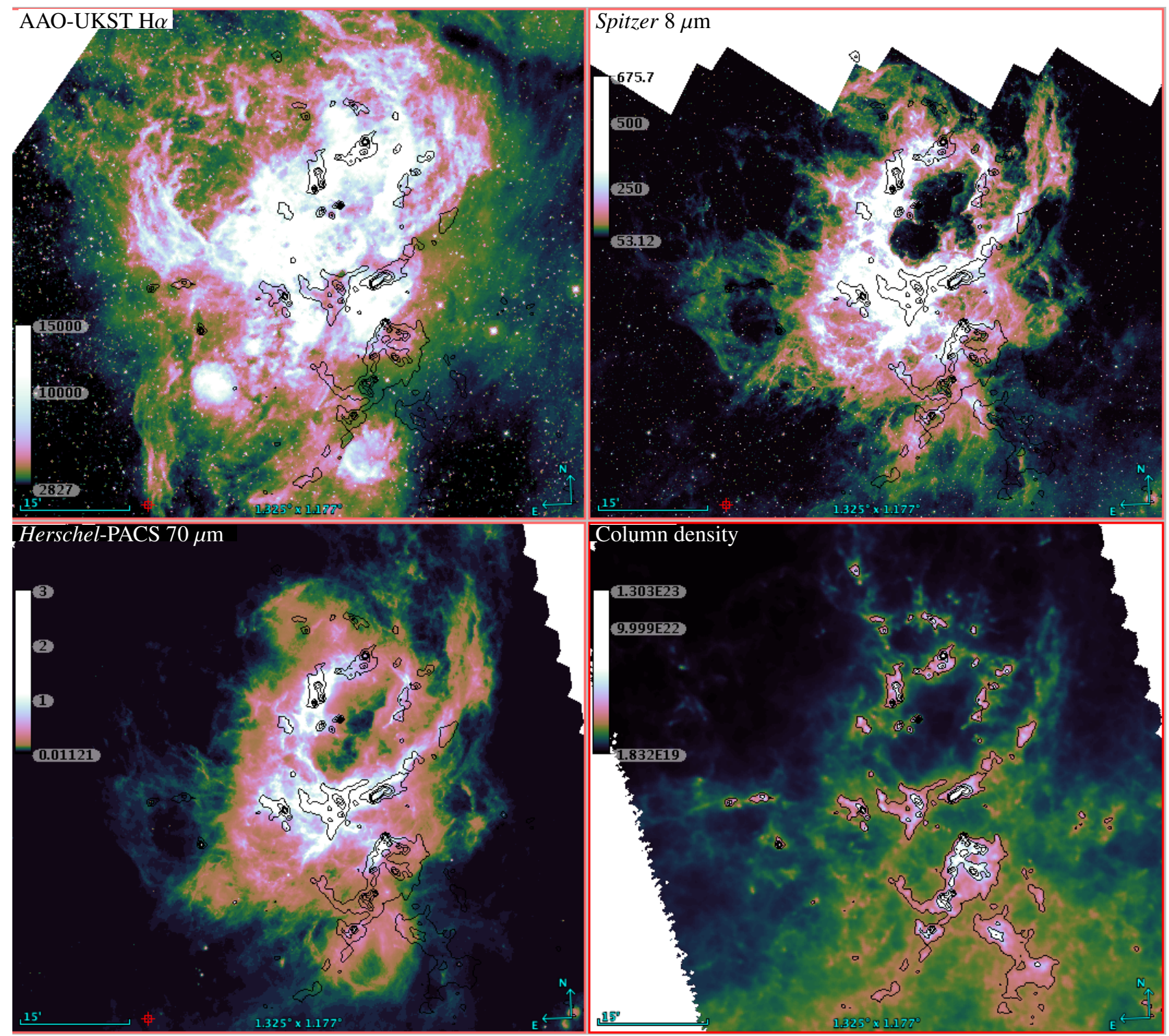

Fig. 2. $\mathrm{H} \alpha$ (arbitrary units), Spitzer $8 \mu \mathrm{m}\left(\mathrm{MJy} \mathrm{sr}^{-1}\right)$, Herschel/PACS $70 \mu \mathrm{m}\left(\mathrm{Jy} \mathrm{pixel}^{-1}\right)$ and Column density $\left(\mathrm{cm}^{-2}\right)$ images of NGC 6357 overlain with column density iso-contours. The field is oriented following the Galactic coordinates and its size is $1.32^{\circ} \times 1.18^{\circ}$.

The dust opacity law used $\left(\kappa_{0}=0.1 \times(v / 1000 \mathrm{GHz})^{2} \mathrm{~cm}^{2} \mathrm{~g}^{-1}\right)$ is similar to that of Hildebrand (1983) with $\beta=2$ and assuming a gas-to-dust ratio of 100 (this dust opacity law is commonly adopted in the other HOBYS papers).

Figures 1 and 2 present $\mathrm{H} \alpha$, Spitzer $8 \mu \mathrm{m}$, Herschel/PACS $70 \mu \mathrm{m}$ and column density images of NGC 6357. While the 8 (PAHs) and $70 \mu \mathrm{m}$ (emitted from warm dust) emissions show large and extended filaments and delineate cavities, the $\mathrm{H} \alpha$ (ionised gas) emission suggests that they are filled by ionised gas. The column density map appears to be more patchy here than in the case of NGC 6334.

\subsection{Ancillary data}

We complemented our Herschel-HOBYS observations with submillimetre and mid-infrared data (see Table 1). The ATLASGAL survey $^{4}$ (APEX Telescope Large Area Survey of the GALaxy, Schuller et al. 2009), using the LABOCA/APEX camera at

\footnotetext{
4 Detailed information and reduced images are available at http:// wwW3.mpifr-bonn.mpg.de/div/atlasgal/
}

$870 \mu \mathrm{m}$, covered the NGC 6357 molecular cloud with 19.2" resolution. NGC 6357 was also covered with 24" angular resolution, by dedicated SIMBA/SEST ${ }^{5} 1.2 \mathrm{~mm}$ observations presented by Muñoz et al. (2007) and Russeil et al. (2010). We also used mid-infrared wavelength images from Spitzer/IRAC and MIPS at 3.6-24 $\mu \mathrm{m}$, as part of the GLIMPSE (Benjamin et al. 2003) and MIPSGAL (Carey et al. 2009) surveys ${ }^{6}$.

NGC 6357 was also covered with surveys made by the WISE $^{7}$ and MSX ${ }^{8}$ space observatories (Wright et al. 2010; Egan et al. 2003). WISE provides full-sky images at four mid-infrared bands, notably at $22 \mu \mathrm{m}$ with an angular resolution of $12^{\prime \prime}$. The Midcourse Space Experiment (MSX) surveyed the Galactic plane in four mid-infrared broad bands, including $21.3 \mu \mathrm{m}$, with $18.3^{\prime \prime}$ angular resolution. One should note that, due to its strong

5 SIMBA/SEST was a former bolometer array of the SEST $15 \mathrm{~m}$.

6 Detailed information and reduced images are available at https:// irsa.ipac.caltech.edu/data/SPITZER/docs/

spitzerdataarchives/

7 see http://irsa.ipac.caltech.edu/Missions/wise.html

8 see http://irsa.ipac. caltech.edu/Missions/msx.html 
Table 1. Data description.

\begin{tabular}{ccc}
\hline \hline Data & $\begin{array}{c}\lambda \\
(\mu \mathrm{m})\end{array}$ & $\begin{array}{c}H P B W \\
\left({ }^{\prime \prime}\right)\end{array}$ \\
\hline \multirow{2}{*}{ Herschel/PACS } & 70 & 5.9 \\
& 160 & 11.7 \\
and SPIRE & 250 & 18.2 \\
& 350 & 24.9 \\
& 500 & 36.3 \\
\hline APEX/LABOCA & 870 & 19.2 \\
\hline SEST/SIMBA & 1200 & 24.0 \\
\hline \multirow{3}{*}{ Spitzer/IRAC } & 3.6 & 1.5 \\
& 4.5 & 1.7 \\
and MIPS & 8 & 2.7 \\
& 24 & 6.0 \\
\hline WISE & 22 & 12.0 \\
\hline MSX & 21 & 18.3 \\
\hline
\end{tabular}

emission, the central part of NGC 6357 is saturated on both MIPS $24 \mu \mathrm{m}$ and WISE $22 \mu \mathrm{m}$ images.

In addition, we retrieved public JCMT-HARP-ACSIS ${ }^{9}$ reduced datacubes of NGC 6357 (proposal M14AU32, PI J. Wouterloot). During this run, the central part of NGC 6357 $\left(31.8^{\prime} \times 28.1^{\prime}\right)$ was observed at $345.795 \mathrm{GHz}\left({ }^{12} \mathrm{CO}(3-2)\right)$ and $330.587 \mathrm{GHz}\left({ }^{13} \mathrm{CO}(3-2)\right)$ and automatically reduced using ORAC-DR (Jenness et al. 2015). The ${ }^{12} \mathrm{CO}(3-2)$ and ${ }^{13} \mathrm{CO}(3-2)$ lines datacubes have respectively 1884 and 1852 channels with a velocity resolution of 0.42 and $0.44 \mathrm{~km} \mathrm{~s}^{-1}$. The beam FWHM is $14^{\prime \prime}$ and the pixel scale is $7.27^{\prime \prime}$ pixel $^{-1}$. In parallel, we collected the 93 MALT $90^{10}$ datacubes pointing in the direction of NGC 6357. MALT90 (Jackson et al. 2013; Foster et al. 2013) is a survey of 2000 dense cores located in the Galactic plane led with the ATNF Mopra 22-m telescope. The database provides $3^{\prime} \times 3^{\prime}$ datacubes for 16 lines simultaneously observed around $90 \mathrm{GHz}$ with an angular and spectral resolution of $38^{\prime \prime}$ and $0.11 \mathrm{~km} \mathrm{~s}^{-1}$, respectively. We complemented the above data with ATNF Mopra 22-m telescope observations (on the fly mapping) of the lines $\mathrm{HCO}^{+}(89.188 \mathrm{GHz})$ and $\mathrm{N}_{2} \mathrm{H}^{+}(93.174 \mathrm{GHz})$ observed in 2008 (reduced with Livedata and Gridzilla codes). The angular and spectral resolution are $30^{\prime \prime}$ and $0.11 \mathrm{~km} \mathrm{~s}^{-1}$, respectively.

\section{Building the massive dense core catalogue}

\subsection{Compact source extraction}

The compact sources were extracted using getsources (v1.140127; Men'shchikov et al. 2012; Men'shchikov 2013). Before running getsources, background subtracted and flattened detection images were produced using getimages (Men'shchikov 2017). getsources first performs the compact source detection, and then measures the fluxes.

The detection module of getsources was run on the Herschel flux maps as well as on the high-resolution column density map. At this step the used PACS-160 $\mu \mathrm{m}$ and SPIRE- $250 \mu \mathrm{m}$ images are the temperature-corrected ones (see

\footnotetext{
9 http://www.cadc-ccda.hia-iha.nrc-cnrc.gc.ca/

${ }^{10}$ http://malt90.bu.edu/index.html
}

Tigé et al. 2017) helping getsources to identify the compact sources against the strong background emission. During this detection step getsources defines a catalogue of sources with a unique position.

For the measurement step, we used the original (not temperature-corrected) Herschel maps from 70 to $500 \mu \mathrm{m}$ plus the available sub-millimetre maps listed in Sect. 2.2, that is, the $870 \mu \mathrm{m}$ LABOCA and $1.2 \mathrm{~mm}$ SIMBA images. At this step, getsources derives flux measurements that are background subtracted and deblended from overlapping sources. The output source catalogue lists, among others, the monochromatic detection significance index ${ }^{11}\left(\mathrm{Sig}_{\text {mono }}\right)$, peak and integrated fluxes (with errors), FWHM major and minor sizes, and the position angle of the elliptical footprint for each extracted source in each far-infrared to sub-millimetre band.

\subsection{Compact source selection}

The Herschel-HOBYS imaging of NGC 6357 extends in Galactic latitude from approximately $-0.4^{\circ}$ to $+1.4^{\circ}$ (see Fig. 1). Russeil et al. (2016) note that sources below $b \sim+0.1^{\circ}$ (the yellow dashed line in Fig. 1) are Galactic plane sources not related to NGC 6357. By masking this area, we collected 922 (out of the 1391 sources located on the whole field) sources in the direction of NGC 6357 from the getsources catalogue.

In order to keep only reliable flux measurements and compact structures, and to be able to subsequently perform the SED fitting, we applied additional source selection criteria as described and discussed in Tigé et al. (2017). We summarise these selection criteria below.

- For each source and each band:

- The signal-to-noise ratio (S/N) must be greater than two (limiting false detections while allowing the SEDs to be well constrained by many flux measurements) for both the peak and integrated fluxes ${ }^{12}$;

- The deconvolved size must be less than $0.3 \mathrm{pc}$ (to discard clumps);

- The aspect ratio must be smaller than two (to discard elongated, filament-like features).

This first step selection process discards $41 \%$ of the sources. They are mainly sources with low signal-to-noise fluxes hence sources at the limit of the detection level in Herschel wavelengths and corresponding to dense cores with very low mass. We can note that only $12(1.3 \%)$ sources have been discarded because of the size criterion.

- For the SED fitting we require a minimum of three reliable flux measurements:

- One at either Herschel-160 $\mu \mathrm{m}$ or Herschel-250 $\mu \mathrm{m}$, which we call the reference wavelength;

- A second Herschel flux measurement at $\lambda \geq 250 \mu \mathrm{m}$;

- A third flux measurement taken at $\lambda>250 \mu \mathrm{m}$ with either

Herschel-SPIRE, APEX/LABOCA, or SEST/SIMBA.

From this second step selection process, $37 \%$ of the sources are discarded because they have no reliable fluxes at Herschel$160 \mu \mathrm{m}$ and/or Herschel-250 $\mu \mathrm{m}$. These sources are either PDR or elongated features. Indeed, dense cores should have SED peaking between 100 and $300 \mu \mathrm{m}$ and should therefore have reliable flux measurements at Herschel-160 $\mu \mathrm{m}$ and/or Herschel $-250 \mu \mathrm{m}$. Finally, 5\% are excluded because they are

11 The detection significance refers to a single-scale analogue to a classical signal-to-noise ratio $(\mathrm{S} / \mathrm{N})$ (see Eq. (17) of Men'shchikov et al. 2012).

12 These criteria correspond to: $S^{\text {peak }} / \sigma>2, S^{\text {int }} / \sigma>2$, and $\operatorname{Sig}_{\text {mono }}>5$. 
$70 \mu \mathrm{m}$-only sources. This gives us a sub-sample of 155 robust sources which fullfill these selection criteria.

\subsection{Compact sources physical characterisation}

The SED fitting was made for the 155 selected sources to determine their mass and temperature (see Table C.1). To construct SEDs with fluxes measured within a similar aperture, we applied the flux scaling procedure and colour correction as described in Tigé et al. (2017). The study of the profile of each core is beyond the scope of this paper. As a result, a flux scaling was performed assuming that sources have a quasi-spherical radial density distribution following a $\rho(r) \sim r^{-2}$ law as it is observed for protostellar envelopes (e.g. Beuther et al. 2002; Nguyen Luong et al. 2011a). This flux scaling allows to reconcile Herschel fluxes with higher resolution observations and has been explained at length in for example Nguyen Luong et al. (2011a) and Tigé et al. (2017). We stress that it has a relatively weak impact (at most $50 \%$ decrease in mass) on the results.

The fitted model is a modified black-body model with a dust emissivity spectral index, $\beta$, set to 2 and the $70 \mu$ m flux is not used for the SED fitting except when an extreme temperature (larger than $32 \mathrm{~K}$ ) is found (Tigé et al. 2017). Indeed, Men'shchikov (2016) demonstrated that mass derivation with a free variable spectral index leads to very strong biases and erroneous masses.

The flux uncertainties were evaluated as in Tigé et al. (2017) and the uncertainties produced by the fitting routine (similar to Tigé et al. 2017) allow us to obtain the mass and temperature uncertainties (their mean value being 35 and $11 \%$, respectively). The core's mass and temperature are obtained by fitting the equation:

$S_{v}=\frac{\operatorname{Mass} \kappa_{v} B_{v}\left(T_{\text {dust }}\right)}{d^{2}}$,

where $S_{v}$ is the dust continuum emission, $d$ is the distance to the Sun, and $\kappa_{v}$ the dust mass opacity (the chosen value of $\kappa_{v}$ is discussed in Tigé et al. 2017). The volume-averaged density is then calculated (as in Tigé et al. 2017) by

$\left\langle n_{\mathrm{H}_{2}}\right\rangle=\frac{\text { Mass }}{\frac{4}{3} \pi \mu \mathrm{m}_{\mathrm{H}}(\operatorname{size} / 2)^{3}}$,

where $\mu=2.8$ and the size is either the deconvolved equivalent FWHM measured at the reference wavelength or, for sources unresolved at 160 or $250 \mu \mathrm{m}$, it is defined ${ }^{13}$ as $0.5 \times H P B W_{\operatorname{Ref} \lambda}$.

Cross-matching (cone search $7^{\prime \prime}$ ) with GLIMPSE 3.6-8 $\mu \mathrm{m}$, MIPSGAL $24 \mu \mathrm{m}$, WISE $22 \mu \mathrm{m}$, and MSX $21 \mu \mathrm{m}$ catalogues $^{14}$ was performed to complement the SED in the mid-IR and nearIR ranges. In addition, counterpart with GLIMPSE sources with rising fluxes between 3.6 and $8 \mu \mathrm{m}$ are favoured, since the $1-$ $10 \mu \mathrm{m}$ SED portion is expected to be rising for both Class I and II young stellar objects (YSOs) (Whitney et al. 2004; Molinari et al. 2008).

This allowed us to compute the bolometric luminosity $\left(L_{\mathrm{bol}}\right)$. We first computed the flux density integration over the finite number of reliable data-points (from the first available data-point

\footnotetext{
$\overline{13}$ For unresolved sources at $160 \mu \mathrm{m}$ or $250 \mu \mathrm{m}$, their upper limit size of 5.85" (resp. 9.1") leads to lower limits for their volume-averaged density.

14 The catalogues (GLIMPSE (I + II + 3D), MIPSGAL, WISE All-Sky Source, and MSXPSC v2.3) can be found via http://irsa.ipac. caltech.edu/applications/Gator/
}

Table 2. Main physical properties of the 155 dense cores in NGC 6357.

\begin{tabular}{lccc}
\hline \hline & Min & Median & Max \\
\hline$F W H M_{\text {Dec }}(\mathrm{pc})$ & 0.05 & 0.08 & 0.29 \\
$\left\langle T_{\text {dust }}\right\rangle(\mathrm{K})$ & 12.1 & 18.8 & 44.9 \\
$L_{\text {bol }}\left(L_{\odot}\right)$ & 4.5 & 203 & $1.210^{4}$ \\
Mass $\left(M_{\odot}\right)$ & 0.5 & 22.2 & 385.8 \\
$\left\langle n_{\mathrm{H}_{2}}\right\rangle\left(\mathrm{cm}^{-3}\right)$ & $2.9 \times 10^{4}$ & $7.8 \times 10^{5}$ & $\geq 1.1 \times 10^{7}$ \\
\hline
\end{tabular}

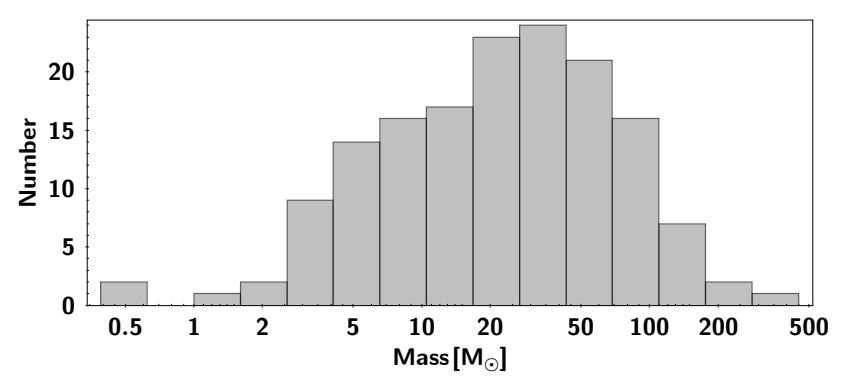

Fig. 3. Mass distribution of the 155 robust sources of NGC 6357.

measurement to the last one in the $3.6 \mu \mathrm{m}$ to $1.2 \mathrm{~mm}$ range) sampling the SED $\left(L_{\text {Data }}\right)$ and using the trapezoid rule (requiring a linear interpolation in the flux density versus frequency space). The data-points which are upper limits are not used in this fitting process. In parallel, we calculated the flux integration in the same way but under the full fitted curve $\left(L_{\mathrm{Fit}}\right)$. Similarly, the submillimetre luminosity $\left(L_{\text {sub-mm }}\right)$ is the flux integration under the fitted curve but only from $350 \mu \mathrm{m}$ to $1.2 \mathrm{~mm}$.

When no mid-IR and near-IR counterparts were found $L_{\text {Fit }}$ is a better evaluation of the true bolometric luminosity, then we adopted $L_{\text {bol }}=\max \left(L_{\text {Fit }}, L_{\text {Data }}\right)$ (in general $\left.L_{\text {Fit }} \geq L_{\text {Data }}\right)$. When mid-IR and/or near-IR counterparts are found the trapezoid integration method is favoured and then $L_{\mathrm{bol}}=L_{\text {Data }}$. However, we must keep in mind that $\sim 19 \%$ of the sources fall in saturated areas of the 22 and $24 \mu \mathrm{m}$ images and only five sources have a compact MSX $21 \mu \mathrm{m}$ counterpart. When the $21 / 22 / 24 \mu \mathrm{m}$ flux is missing this leads to an over-estimate of $L_{\text {Data }}$, relative to the true bolometric luminosity, by a factor of between two and four (Tigé 2014). In addition, $L_{\text {Data }}$ must be considered with great caution when the source has near-IR counterparts but no $21 / 22 / 24 \mu \mathrm{m}$ flux and no $70 \mu \mathrm{m}$ flux. In this case the $L_{\text {Data }}$ is unrealistically large because the linear interpolation in the trapezoid integration method implies its strong over-estimation (by a factor of up to ten).

Due to its large uncertainty, $L_{\mathrm{bol}}$ is used no further in this paper and does not affect its conclusions. Table 2 summarises the main properties of the sample of 155 cores and the mass distribution is shown in Fig. 3. The mass distribution (Fig. 3) peaking at $\sim 30 M_{\odot}$, we adopted this value as completeness level.

\section{The complete sample of NGC 6357 MDCs}

\subsection{Mass limit for massive dense cores}

Tigé et al. (2017) established a lower mass limit of $75 M_{\odot}$ to select the massive dense cores (MDCs) in NGC 6334. Due to a poor maser and compact $\mathrm{H}$ II region surveys coverage and strong saturation in mid-infrared images in NGC 6357 we cannot make a proper estimate of this threshold. Therefore, we adopted the same lower mass limit for the MDCs of NGC 6334. This also 
Table 3. Main physical properties of the 23 MDCs in NGC 6357.

\begin{tabular}{cccc}
\hline \hline & Min & Median & Max \\
\hline$F W H M_{\text {Dec }}(\mathrm{pc})$ & $0.07(0.05)$ & $0.13(0.08)$ & $0.29(“ 0.30 ”)$ \\
$\left\langle T_{\text {dust }}\right\rangle(\mathrm{K})$ & $12.1(9.5)$ & $16.7(16.7)$ & $25.2(40.1)$ \\
$L_{\text {bol }}\left(L_{\odot}\right)$ & $24(10)$ & $265(320)$ & $2.4 \times 10^{3}\left(8.7 \times 10^{4}\right)$ \\
Mass $\left(M_{\odot}\right)$ & "75"(“75”) & $102(120)$ & $386(1020)$ \\
$\left\langle n_{\mathrm{H}_{2}}\right\rangle\left(\mathrm{cm}^{-3}\right)$ & $1.2 \times 10^{5}\left(1 \times 10^{5}\right)$ & $1.3 \times 10^{6}\left(6 \times 10^{6}\right)$ & $\geq 6.2 \times 10^{6}\left(\geq 7 \times 10^{7}\right)$ \\
\hline
\end{tabular}

Notes. Values in italic and into parenthesis are for NGC 6334, from Tigé et al. (2017). Values in quotes are lower or upper limits due to the selection process.

allowed us to make an homogeneous comparison between the two regions. by looking for signposts of high-mass star formation. Nevertheless, to validate this choice we look for signs of high-mass star formation by cross-correlating (cone search $10^{\prime \prime}$ ) the 155 robust sources with Class II $\mathrm{CH}_{3} \mathrm{OH}$ masers (Caswell et al. 2010; Urquhart et al. 2013) and radio centimeter UCH II regions (White et al. 2005; Condon et al. 1998; Giveon et al. 2005). We find maser or H II region association for only seven cores. However four cores have a methanol maser with velocity peaking at $-51,-41$ or $-16 \mathrm{~km} \mathrm{~s}^{-1}$ respectively suggesting they do not belong to NGC 6357 (the systemic LSR velocity of the region is $-4 \mathrm{~km} \mathrm{~s}^{-1}$, Caswell \& Haynes 1987) and two cores have compact radio counterpart, but their masses are low $\left(M \leq 10 M_{\odot}\right)$ suggesting either that their association is erroneous or they are $\mathrm{H}$ II regions at different distances along the NGC 6357 line of sight. Only one core exhibits a $\mathrm{CH}_{3} \mathrm{OH}$ maser and a strong and compact MSX $21 \mu \mathrm{m}$ emission (it is in a saturated area on MIPSGAL $24 \mu \mathrm{m}$ and WISE $22 \mu \mathrm{m}$ images). In addition this core is classified as an "extended green object" (an object within an enhanced Spitzer $4.5 \mu \mathrm{m}$ emission usualy attributed to shock exited $\mathrm{H}_{2}$ features tracing outflow, Cyganowski et al. 2008) by Chambers et al. (2014). Assuming the evolutionnary tracks from Molinari et al. (2008), the evolved nature of this core, with a present mass of $48 M_{\odot}$, could suggest an earlier mass around $140 M_{\odot}$ consistent with the adopted mass limit.

For NGC 6357, we finally obtain a selection of 23 MDCs. Table 3 gives their main physical properties while their fluxes, multi-wavelength images and SEDs are displayed in Appendixes $\mathrm{C}$ and $\mathrm{D}$. Because the mass limit $\left(75 M_{\odot}\right)$ is well above the completeness level, we assume that we do not miss any MDC in NGC 6357. At a first look, we note that MDCs in NGC 6357 are slightly larger and less dense and massive than those in NGC 6334.

\subsection{Nature and evolutionary status of MDCs}

To estimate the evolutionary status of MDCs we followed a similar approach to that in Motte et al. (2007), Csengeri et al. (2014), König et al. (2017), Giannetti et al. (2017), and Tigé et al. (2017). We classified the MDCs into IR-bright protostellar MDC, IR-quiet protostellar MDC, starless MDC or undefined cloud. An MDC is qualified as an "IR-bright protostellar" if it has a 21 , 22 , or $24 \mu \mathrm{m}$ flux larger than 10, 12, $15 \mathrm{Jy}$, respectively (for $d=1.75 \mathrm{kpc}$, Motte et al. 2010; Russeil et al. 2010), a maser and/or H II region (radio continuum) association and a clear centrally-located Spitzer $-8 \mu \mathrm{m}$ point source. But, because of the large area saturared at 22 and $24 \mu \mathrm{m}$ and due to the poor coverage and quality of the radio and maser surveys it is difficult to find sources fullfilling all these criteria and we can miss IR-bright MDCs identification. Despite relaxing the IR-bright protostellar status to sources fullfilling at least one of these criteria we found no IR-bright protostellars MDC. As in Tigé et al. (2017), an MDC is qualified as an IR-quiet protostellar if it is associated with a $70 \mu \mathrm{m}$ compact emission even if it is detected or not at 21/22/24 $\mu \mathrm{m}$ (but lower than 10/12/15 Jy).

An MDC is qualified as "starless" MDC candidate if no compact $70 \mu \mathrm{m}$ and no $21 / 22 / 24 \mu \mathrm{m}$ emission are detected, in addition to be centrally concentrated ${ }^{15}$ If it is not centrally concentrated the MDC is then qualified as an "undefined cloud structure" (corresponding to unbound cloud structures). The qualification of undefined cloud structures was introduced by Tigé et al. (2017) and Rayner et al. (2017). These are cloud structures that are not centrally concentrated. To estimate the MDCs detection reliability we check whether they are also in the Hi-GAL (Molinari et al. 2016) source catalogue obtained with the $\mathrm{CuTEx}^{16}$ algorithm. The two MDCs (see Table 4) straddling two Hi-GAL sources are undefined cloud structures.

The final sample of MDCs is plotted on Fig. 4. We note that they are located on high column density regions and they are mainly distributed along a strip close to $l \sim 353.1^{\circ}$.

\subsection{Velocity structure and massive dense cores properties}

From the ${ }^{12} \mathrm{CO}(3-2)$ integrated profile a systemic velocity for the region of $-3.5 \mathrm{~km} \mathrm{~s}^{-1}$ is measured, but several velocity components can be seen (Fig. 5). The $-4 \mathrm{~km} \mathrm{~s}^{-1}$ component is well correlated with the column density, delineating the cavity that is clearly filled by ionised gas. In the direction of the cavity seen by Russeil et al. (2016), the ionised gas ( $\mathrm{H} \alpha$ emission) shows a velocity of $-2 \mathrm{~km} \mathrm{~s}^{-1}$ in the central part while the surroundings consist of an $\mathrm{H} \alpha$ semi-ring-like feature at $-8 \mathrm{~km} \mathrm{~s}^{-1}$, to which the region G353.2+0.9 Giannetti et al. 2012 belongs. A few features are found around between -9 and $-12 \mathrm{~km} \mathrm{~s}^{-1}$. They are pillar-like features at $l, b=353.16^{\circ},+0.82^{\circ}(\alpha, \delta=17 \mathrm{~h} 24 \mathrm{~m} \mathrm{55s}$, $\left.-34^{\circ} 13^{\prime \prime}\right)$ pointing towards the cavity centre and the elongated feature at $l, b=353.09^{\circ},+0.71^{\circ}\left(\alpha, \delta=17 \mathrm{~h} 25 \mathrm{~m} 15 \mathrm{~s},-34^{\circ} 22^{\prime}\right)$. Both are located, in projection, inside the cavity and are seen in absorption on the $\mathrm{H} \alpha$ image suggesting they are at the front edge of the region. At $l, b=353.15^{\circ},+0.67^{\circ}(\alpha, \delta=17 \mathrm{~h} 25 \mathrm{~m} \mathrm{35s}$, $\left.-34^{\circ} 20^{\prime}\right)$ this $-9 \mathrm{~km} \mathrm{~s}^{-1}$ component is superimposed on the main $-4 \mathrm{~km} \mathrm{~s}^{-1}$ emission. At $+2 \mathrm{~km} \mathrm{~s}^{-1}$ the emission follows the $\mathrm{H} \alpha$ emission around the region $\mathrm{G} 353.2+0.9$, tracing a

\footnotetext{
15 The core concentration on the different Herschel maps was evaluated by looking at the longitudinal and transverse cuts but also by an automated procedure described in Konyves et al. (in prep.). In short, this automated procedure checked directly in the maps whether a core is centrally peaked within its measured FWHM, by evaluating map values under concentric annular masks constructed inside and outside of the FWHM ellipse of a given source at the wavelengths of 70-500 $\mu \mathrm{m}$, and also in the high-resolution column density map.

16 see http://herschel . asdc . asi .it/index . php?page=cutex . html
} 
Table 4. Physical parameters of the MDCs found in NGC 6357.

\begin{tabular}{|c|c|c|c|c|c|c|c|c|}
\hline $\begin{array}{l}\text { MDC } \\
\text { Id. }\end{array}$ & $\begin{array}{c}\mathrm{MDC}^{(a)} \\
\mathrm{nb} .\end{array}$ & $\begin{array}{c}F W H M_{\text {Dec }} \\
(\mathrm{pc})\end{array}$ & $\begin{array}{c}\left\langle T_{\text {dust }}\right\rangle \\
(\mathrm{K})\end{array}$ & $\begin{array}{l}\text { Mass } \\
\left(M_{\odot}\right)\end{array}$ & $\begin{array}{r}L_{\text {bol }} \\
\left(L_{\odot}\right) \\
\end{array}$ & $\begin{array}{c}\left\langle n_{\mathrm{H}_{2}}\right\rangle \\
\left(\times 10^{6} \mathrm{~cm}^{-3}\right)\end{array}$ & $\begin{array}{c}L_{\text {sub-mm }} / L_{\text {bol }} \\
(\%)\end{array}$ & Comments ${ }^{(b)}$ \\
\hline \multicolumn{9}{|c|}{ IR-quiet protostellar MDC } \\
\hline HOBYS_J172631.1-340236 & 6 & 0.12 & $13.1 \pm 0.3$ & $155.0 \pm 20.3$ & 76 & $2.64 \pm 0.34$ & 10.7 & $70 \mu \mathrm{m}$ compact source \\
\hline \multicolumn{9}{|c|}{ Starless MDC candidate } \\
\hline HOBYS_J172516.5-342446 & 1 & 0.21 & $17.0 \pm 0.6$ & $385.8 \pm 50.7$ & 905 & $1.19 \pm 0.15$ & 4.3 & \\
\hline HOBYS_J172535.8-342051 & 5 & 0.25 & $23.2 \pm 1.3$ & $160.5 \pm 29.2$ & 2420 & $0.28 \pm 0.05$ & 1.3 & \\
\hline HOBYS_J172601.1-342955 & 7 & 0.26 & $20.3 \pm 2.3$ & $137.2 \pm 37.2$ & 919 & $0.22 \pm 0.05$ & 2.2 & \\
\hline HOBYS_J172412.3-341307 & 10 & 0.13 & $15.3 \pm 0.7$ & $111.6 \pm 22.0$ & 139 & $1.45 \pm 0.28$ & 6.3 & \\
\hline HOBYS_J172446.2-341048 & 11 & 0.08 & $23.3 \pm 1.5$ & $103.2 \pm 21.1$ & 1568 & $6.19 \pm 1.27$ & 1.2 & \\
\hline HOBYS_J172538.8-343058 & 12 & 0.10 & $14.9 \pm 1.0$ & $102.4 \pm 28.0$ & 107 & $3.05 \pm 0.83$ & 6.9 & \\
\hline HOBYS_J172357.2-340545 & 14 & 0.25 & $15.6 \pm 0.5$ & $97.5 \pm 14.7$ & 168 & $0.17 \pm 0.02$ & 4.4 & No Hi-GAL cov. \\
\hline HOBYS_J172451.0-341018 & 17 & 0.08 & $25.3 \pm 2.2$ & $83.1 \pm 19.5$ & 2065 & $4.98 \pm 1.17$ & 0.9 & \\
\hline HOBYS_J172350.5-340813 & 18 & 0.12 & $15.9 \pm 0.6$ & $82.2 \pm 13.8$ & 125 & $1.29 \pm 0.21$ & 5.6 & No Hi-GAL cov. \\
\hline HOBYS_J172623.1-343223 & 21 & 0.15 & $16.7 \pm 2.1$ & $80.6 \pm 28.7$ & 167 & $0.60 \pm 0.21$ & 4.6 & \\
\hline HOBYS_J172548.0-342852 & 22 & 0.08 & $16.2 \pm 2.4$ & $79.0 \pm 40.3$ & 138 & $3.77 \pm 1.92$ & 5.1 & \\
\hline HOBYS_J172459.2-341427 & 23 & 0.13 & $18.7 \pm 1.0$ & $75.4 \pm 14.5$ & $431^{(c)}$ & $0.85 \pm 0.16$ & 2.2 & \\
\hline \multicolumn{9}{|c|}{ Undefined cloud structure } \\
\hline HOBYS_J172519.7-342427 & 2 & 0.13 & $21.2 \pm 2.4$ & $237.1 \pm 62.5$ & 2076 & $2.84 \pm 0.75$ & 1.8 & \\
\hline HOBYS_J172422.4-341218 & 3 & 0.29 & $15.7 \pm 1.7$ & $182.1 \pm 58.0$ & 265 & $0.20 \pm 0.06$ & 1.0 & Over two Hi-GAL \\
\hline HOBYS_J172410.3-340216 & 4 & 0.20 & $12.9 \pm 1.3$ & $163.1 \pm 56.9$ & 72 & $0.58 \pm 0.20$ & 11.4 & \\
\hline HOBYS_J172439.6-340924 & 8 & 0.12 & $20.1 \pm 1.2$ & $134.2 \pm 27.5$ & 847 & $2.14 \pm 0.44$ & 2.3 & Over two Hi-GAL \\
\hline HOBYS_J172646.5-343102 & 9 & 0.30 & $16.3 \pm 1.5$ & $111.9 \pm 29.2$ & 201 & $0.11 \pm 0.03$ & 2.2 & \\
\hline HOBYS_J172350.7-340726 & 13 & 0.25 & $15.7 \pm 1.7$ & $98.9 \pm 33.3$ & 145 & $0.16 \pm 0.05$ & 5.6 & No Hi-GAL cov. \\
\hline HOBYS_J172444.3-341031 & 15 & 0.08 & $24.9 \pm 1.7$ & $93.0 \pm 18.7$ & 2122 & $4.84 \pm 0.97$ & 0.6 & \\
\hline HOBYS_J172539.3-342839 & 16 & 0.09 & $18.2 \pm 0.9$ & $89.8 \pm 15.5$ & 308 & $3.46 \pm 0.59$ & 3.4 & \\
\hline HOBYS_J172635.6-340230 & 19 & 0.12 & $12.1 \pm 0.6$ & $81.1 \pm 17$ & 24 & $1.32 \pm 0.28$ & 13.9 & \\
\hline HOBYS_J172515.1-340940 & 20 & 0.20 & $21.5 \pm 3.1$ & $80.8 \pm 27.6$ & 769 & $0.26 \pm 0.08$ & 1.7 & \\
\hline
\end{tabular}

Notes. ${ }^{(a)}$ The MDC's numbering is done by decreasing mass. ${ }^{(b)}$ All MDCs are associated with a Hi-GAL source (Molinari et al. 2016) except when it is indicated as "No Hi-GAL cov". (not covered by the Hi-GAL survey) or "Over two Hi-GAL" (meaning that the MDC is straddling two Hi-GAL sources). ${ }^{(c)}$ For MDC \#23, the lack of mid-IR and $70 \mu \mathrm{m}$ fluxes make a large and unreliable over-estimation of $L_{\mathrm{bol}}$. Then the quoted $L_{\mathrm{bol}}$ is assigned to be $L_{\text {Data }}$ but calculated using the expected $70 \mu \mathrm{m}$ flux evaluated from the fitted curve.

semi-continuous elliptical-like feature of $6.7^{\prime} \times 8.3^{\prime}$ size, centred at $l, b=353.08^{\circ},+0.83^{\circ}\left(\alpha, \delta=17 \mathrm{~h} 24 \mathrm{~m} 45.7 \mathrm{~s},-34^{\circ} 18^{\prime} 21.9^{\prime \prime}\right)$, while it is found towards $\mathrm{H} \alpha$ extinction areas and superimposed on the $-4 \mathrm{~km} \mathrm{~s}^{-1}$ emission around $l, b=353.15^{\circ},+0.67^{\circ}$. Therefore, this position appears to be special due to the different velocity components mixing and extended profiles. This can be due to a combination of component superposition and self-absorption effects. However, this place corresponds to the contact zone between the cavity and the regions H II $353.09+0.63$ and H II 353.24+0.60 (Russeil et al. 2016) where dynamical interaction of the ionised gas with the molecular cloud can occur. There are also young stars and OB stars (Getman et al. 2014; Russeil et al. 2017) which can participate to the local turbulence by their feedback. Assuming that the -9 and the $+2 \mathrm{~km} \mathrm{~s}^{-1}$ are the extreme velocities and $-4 \mathrm{~km} \mathrm{~s}^{-1}$ the systemic velocity, we can estimate an expansion velocity of $\sim 5 \mathrm{~km} \mathrm{~s}^{-1}$ for the NGC 6357 central cavity. Finally, the patchy $+6 \mathrm{~km} \mathrm{~s}^{-1}$ emission is usually attributed to a foreground layer (Russeil et al. 2017).

We have extracted the spectra ${ }^{17}$ at the position of 20 among 23 MDCs in order to measure the core's local environment velocity and to identify particular profile. The $\mathrm{N}_{2} \mathrm{H}^{+}$spectra

17 To increase the signal-to-noise ratio the spectra were extracted from $1.26^{\prime}$ to $1^{\prime}$ area for MALT90 and Mopra data, respectively. are fitted using the hyperfine fitting routine of the spectroscopic analysis toolkit "pyspeckit v0.1.20" (Ginsburg \& Mirocha 2011) assuming a single temperature. However, for lot of $\mathrm{N}_{2} \mathrm{H}^{+}$spectra the modelled profile over- or under-estimate some of the hyperfine components suggesting that it is not consistent with a single temperature assumption. The $\mathrm{HCO}^{+}$spectra are fitted by simple Gaussian(s).

The optically thick $\mathrm{HCO}^{+}$line is usualy used to probe collapse or outflows (e.g. Fuller et al. 2005) as a double peaked profile with an excess of emission on the blue (red) side is generally interpreted as an indication of collapse or infall (outflow). In parallel, combining $\mathrm{HCO}^{+}$and $\mathrm{N}_{2} \mathrm{H}^{+}$, considered as optically thick and thin lines respectively, we can calculate the asymmetry parameter $\delta v$, as defined by Mardones et al. (1997), which indicates a clear asymmetry if $|\delta v|>0.25$.

The results are listed in Table 5. The MDCs show a mean velocity of $-3.74 \mathrm{~km} \mathrm{~s}^{-1}$, while the MDC \#23 has a velocity approximately $-10 \mathrm{~km} \mathrm{~s}^{-1}$ (in agreement with its location in the pillar like feature previously noted at $l, b=353.16^{\circ},+0.82^{\circ}$ ). For the IR-quiet protostellar MDC the spectral analysis is in agreement with its proto-stellar classification as it shows a double-peaked asymetric $\mathrm{HCO}^{+}$profile. However its $\mathrm{HCO}^{+}$blue and red peaks relative intensity suggest an outflow while the asymmetry factor suggests an infall motion. For starless MDCs, 


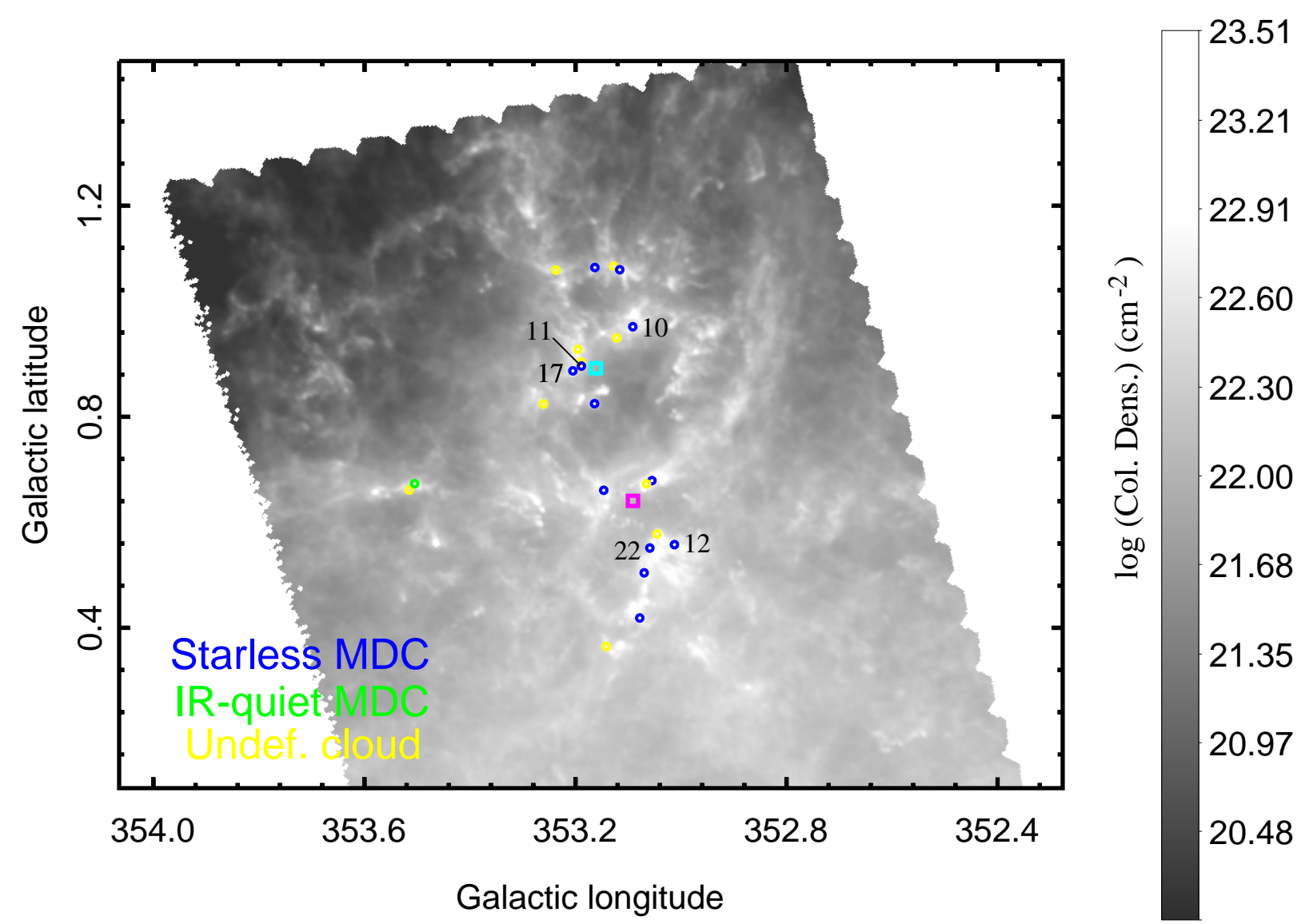

Fig. 4. NGC 6357 column density $\left(N_{\mathrm{H}_{2}}\right)$ map overlain with the evolutionary colour-coded sub-samples of MDCs (MDCs 10, 11, 12, 17, and 22 are indicated). The cyan and magenta diamonds indicate the position of the OB clusters Pismis 24 and AH03J1725-34.4, respectively.

four have $|\delta v|>0.25$ while for undefined clouds none of them have a significant asymmetry. Interestingly, the starless MDC \#23 has the most negative $\delta v$ (suggesting collapse), and probably is in a slightly more evolved stage than the other starless MDCs.

To estimate the stability of the MDCs, virial parameter (defined as $\alpha_{\mathrm{vir}}=M_{\mathrm{vir}} / M_{\mathrm{MDC}}$ by for example, Bertoldi $\&$ McKee 1992 ) is calculated. For this, from the velocity width of the $\mathrm{N}_{2} \mathrm{H}^{+}$ we derive the turbulent component as $\sigma_{\text {turb }}^{2}=\sigma_{\mathrm{N}_{2} \mathrm{H}^{+}}^{2}-\sigma_{\text {th }}^{2}$ where $\sigma_{t h}$ is calculated from the dust temperature. The virial mass and the virial parameter are calculated using $\sigma_{\text {turb }}$. These quantities are reported in Table 5, for MDCs with no $\mathrm{N}_{2} \mathrm{H}^{+}, \sigma_{\text {turb }}$ and $\alpha_{\text {vir }}$ are calculated from the $\mathrm{HCO}^{+}$line. The virial parameter can be used for estimating whether a structure is in a state of being gravitationally bound. In this way, we are able to probe the virial equilibrium for most of the MDCs finding that 14 of them have $\alpha_{\text {vir }} \leq 1$, suggesting they are gravity dominated (and may be undergoing collapse), while four have $1<\alpha_{\text {vir }}<2$ suggesting they are at least gravitationally bound and 1 MDCs has $\alpha_{\text {vir }}>$ 2 suggesting it is unbound and may expand (in agreement with the fact that it is also classified as undefined cloud). On average undefined clouds structures have larger $\alpha_{v i r}$ but most of them have $\alpha_{\text {vir }}<2$, suggesting they are not transient features.

\section{Discussion}

\subsection{Comparison with previous massive dense core studies in NGC 6357}

We have compared our Herschel cores with the 74 clumps $(\sim 0.4 \mathrm{pc})$ extracted at $1.2 \mathrm{~mm}$ by Russeil et al. (2010). We find that 36 clumps have single associated Herschel sources, 11 have two or three associated sources, and 27 have none. Of the 36 clumps associated with a single Herschel core, ten are associated with a $M>75 M_{\odot}$ cores $^{18}$. Among the 11 clumps with two Herschel sources, one encompasses two massive cores, four encompass one massive core and six encompass low mass cores only. The $1.2 \mathrm{~mm}$ clumps with no associated Herschel source always have $M<75 M_{\odot}$ or a size larger than $0.3 \mathrm{pc}$. Inversely, 16 of the $23 \mathrm{MDCs}$ are found in the direction of a $1.2 \mathrm{~mm}$ clump, 13 being located within a $M \geq 100 M_{\odot}$ clump.

Giannetti et al. (2012) studied the molecular cloud associated with the region G353.2+0.9 facing Pismis 24. They distinguished, from molecular lines, 14 clumps (with size between 0.27 and $0.56 \mathrm{pc}$ and a total mass of $\sim 2000 M_{\odot}$ ). From our sample, ten reliable cores are detected in the same area among which four are MDCs and appear located in the Giannetti et al. (2012) clumps of mass between 180 and $360 M_{\odot}$. We also note that 18 of our 23 MDCs fall in an ATLASGAL compact source footprint (Csengeri et al. 2014; Urquhart et al. 2018) and one (MDC \#18) partly overlaps an ATLASGAL source.

All this highlights how this study allows us to see the fragmentation process within clumps. This is also in agreement with the results of Csengeri et al. (2017a) who find a limited fragmentation (at the typical scale of $\sim 0.06 \mathrm{pc}$ ) from a sample of 35 massive infrared quiet ATLASGAL clumps. This underlines the importance of the Herschel resolution and multi-wavelength information for the MDCs census and characterisation in comparison to previous studies.

18 A massive dense core is defined as a core with $M>75 M_{\odot}$; see Sect. 4.1. 
D. Russeil et al.: Herschel-HOBYS study of the earliest phases of high-mass star formation in NGC 6357
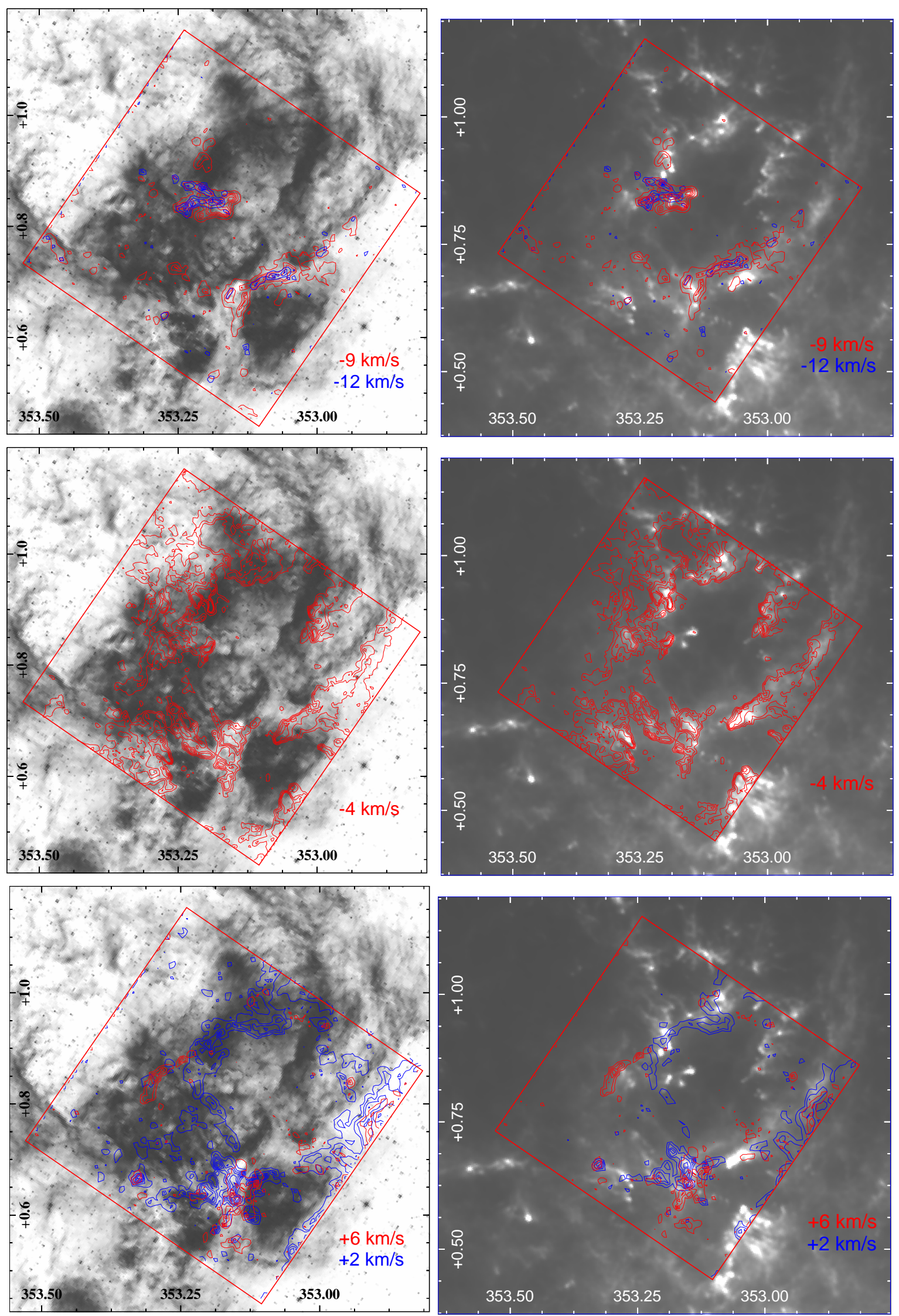

Fig. 5. AAO-UKST H $\alpha$ image (left) and column density map (right) overlaid by JCMT-HARPS ${ }^{12} \mathrm{CO}(3-2)$ emission iso-contours at different velocity $\left(-12,-9,-4,+2\right.$ and $\left.+6 \mathrm{~km} \mathrm{~s}^{-1}\right)$. 
Table 5. Velocity information for the MDCs associated with NGC 6357.

\begin{tabular}{|c|c|c|c|c|c|c|c|c|c|c|c|}
\hline $\begin{array}{l}\text { MDC } \\
\text { Id } \\
(1) \\
\end{array}$ & $\begin{array}{c}V_{\mathrm{LSR}} \\
\text { (primary) } \\
\mathrm{km} \mathrm{s}^{-1} \\
(2)\end{array}$ & $\begin{array}{c}V_{\mathrm{LSR}} \\
\text { (secondary) } \\
\mathrm{km} \mathrm{s}^{-1} \\
(3)\end{array}$ & Line & $\begin{array}{l}\mathrm{HCO}^{+} \\
\text {note } \\
(5)\end{array}$ & $\begin{array}{l}\mathrm{N}_{2} \mathrm{H}^{+} \\
\text {note } \\
(6)\end{array}$ & $\begin{array}{c}V_{\mathrm{N}_{2} \mathrm{H}^{+}} \\
\mathrm{km} \mathrm{s}^{-1} \\
(7)\end{array}$ & $\begin{array}{c}\sigma_{N_{2} H^{+}} \\
\mathrm{km} \mathrm{s}^{-1} \\
(8)\end{array}$ & $\begin{array}{l}\text { Asymmetry } \\
\text { factor } \\
\text { (9) }\end{array}$ & $\begin{array}{c}\sigma_{\text {turb }} \\
\mathrm{km} \mathrm{s}^{-1} \\
(10)\end{array}$ & $\alpha_{\mathrm{vir}}$ & $\begin{array}{c}V_{\mathrm{LSR}} \\
\text { litterature } \\
\mathrm{km} \mathrm{s}^{-1} \\
(12) \\
\end{array}$ \\
\hline \multicolumn{12}{|c|}{ IR-quiet protostellar MDC } \\
\hline 6 & -2.12 & -5.24 & $\mathrm{HCO}^{+}$ & $B \ll R$ & $\operatorname{Reg}$ & -1.74 & 0.62 & -0.26 & 0.57 & 0.15 & -3.1 \\
\hline \multicolumn{12}{|c|}{ Starless MDC candidate } \\
\hline 1 & -4.15 & & $\mathrm{HCO}^{+}$ & G & Reg & -4.17 & 0.88 & +0.01 & 0.84 & 0.23 & -3.5 \\
\hline 5 & -3.53 & & $\mathrm{HCO}^{+}$ & Flat & Faint & -4.44 & 1.09 & +0.36 & 1.05 & 1.03 & -4.4 \\
\hline 7 & -3.40 & & $\mathrm{HCO}^{+}$ & BS & Reg & -3.04 & 0.70 & -0.22 & 0.64 & 0.47 & -3.3 \\
\hline 10 & -4.24 & & $\mathrm{HCO}^{+}$ & G & $\operatorname{Reg}$ & -4.45 & 0.80 & +0.11 & 0.76 & 0.40 & -4.6 \\
\hline 11 & -5.78 & & $\mathrm{HCO}^{+}$ & $\mathrm{G}$ & No det. & & & & 1.05 & 0.49 & -5.1 \\
\hline $\begin{array}{l}12 \\
14\end{array}$ & -2.98 & & $\mathrm{HCO}^{+}$ & $\mathrm{BS}$ & Irr & -1.39 & 1.49 & -0.45 & 1.48 & 1.25 & -1 \\
\hline 17 & -2.76 & & $\mathrm{HCO}^{+}$ & BW & Irr & -2.41 & 0.63 & -0.24 & 0.55 & 0.17 & -2 \\
\hline 18 & & & & & & & & & & & -4 \\
\hline 21 & 2.49 & & $\mathrm{HCO}^{+}$ & $\mathrm{G}$ & Reg & 1.95 & 0.83 & +0.28 & 0.79 & 0.72 & +1.7 \\
\hline 22 & -4.12 & & $\mathrm{HCO}^{+}$ & $\mathrm{RS}$ & Reg & -4.01 & 0.75 & -0.06 & 0.70 & 0.31 & \\
\hline 23 & -10.31 & & $\mathrm{HCO}^{+}$ & RW & $2 \mathrm{P}$ & -10.64 & 0.66 & +0.21 & 0.60 & 0.39 & -10.9 \\
\hline \multicolumn{12}{|c|}{ Undefined cloud structure } \\
\hline 2 & -4.26 & & $\mathrm{HCO}^{+}$ & $\mathrm{G}$ & Reg & -4.18 & 0.91 & -0.03 & 0.87 & 0.25 & -3.5 \\
\hline 3 & -4.23 & & $\mathrm{HCO}^{+}$ & RW? & No det. & & & & 1.08 & 1.11 & +1.4 \\
\hline 4 & -4.84 & & $12 \mathrm{CO}(3-2)^{(a)}$ & & & & & & & & -4.2 \\
\hline 8 & -4.36 & & $\mathrm{HCO}^{+}$ & BWRW & Reg & -4.24 & 0.52 & -0.10 & 0.44 & 0.10 & -4.5 \\
\hline 9 & -1.02 & & $\mathrm{HCO}^{+}$ & G & Irr & -0.92 & 0.5 & -0.09 & 0.43 & 0.30 & \\
\hline 13 & & \multirow{5}{*}{-5.66} & & & & & & & & & \\
\hline 15 & -5.20 & & $\mathrm{HCO}^{+}$ & RW & No det. & & & & 1.47 & 1.15 & -5.1 \\
\hline 16 & -3.42 & & $\mathrm{HCO}^{+}$ & G & $\operatorname{Reg}$ & -3.26 & 0.61 & -0.11 & 0.55 & 0.18 & -3.3 \\
\hline 19 & -1.78 & & $\mathrm{HCO}^{+}$ & $B \gg R$ & Irr & -5.59 & 0.68 & -0.04 & 0.65 & 0.37 & \\
\hline 20 & -4.90 & & $\mathrm{HCO}^{+}$ & G & no det. & & & & 1.38 & 2.91 & -5.4 \\
\hline
\end{tabular}

Notes. Column (4): line used for the velocity measurements given in Cols. 2 and 3. ${ }^{(a)}$ Indicates a poor quality spectrum. Column (5): $\mathrm{HCO}^{+}$line morphology. G: gaussian shape. BW (RW): wing on the blue (red) side. BS (RS): shoulder on the blue (red) side. Irr: irregular shape. $B>R, B<\mathrm{R}$ or $B=R$ : the blue peak higher (smaller or equal) than the red one. Flat: means that it is a flat-top profile. Column (6): "Reg" means a regular hyperfine line structure while "Irr" suggests possible multiple components or low S/N. 2P: two different velocities along the line of sight. Column (12): velocity of the ATLASGAL clump from Urquhart et al. (2018).

\subsection{Quest for the best birthplaces for high-mass stars}

In NGC 6334, Tigé et al. (2017) identified 46 MDCs among which 16 are starless candidates and thus possible sites for the pre-stellar stage of high-mass star formation. However among these 16 starless candidates only one was held as the best candidate, while nine were excluded due to poor SED fitting, and the others due to their low density $\left(1-5 \times 10^{5} \mathrm{~cm}^{-3}\right)$ or because they were not centrally concentrated. Similarly in NGC 6357 we can evaluate the reliability of detected starless MDCs to be truly precursors of high-mass stars. In NGC 6357, except MDC \#1, the SED fitting of the starless MDCs is good $\left(\chi^{2}<5\right)$. In addition, their mass is above the lower limit for high-mass star formation (given e.g. by the mass-radius relation $M(r) \geq 1282 \times(r / p c)^{1.42} M_{\odot}$ from Baldeschi et al. 2017) and their surface density, $\Sigma$, is larger than $0.05 \mathrm{~g} \mathrm{~cm}^{-2}$ (He et al. 2015) suggesting that all the starless MDCs are on first approximation possible sites of high-mass star formation. However, from mass-radius plots the massive-star formation limit is empirical, and based on fits to different clump or core samples. For example, Kauffmann \& Pillai (2010) and Urquhart et al. (2014) find different slope and intercept values for their fitted power-laws than Baldeschi et al. (2017). In addition, as noted by Baldeschi et al. (2017) the massive star formation (MSF) thresholds certainly breaks at mass lower than $20 M_{\odot}$. This is because the adopted values of the core-to-star conversion factor (between 0.5 and 0.33 from Alves et al. 2007) suggest it is not reasonable for a core (if there is no ambient accretion) that will form a high-mass star to have a mass lower than $20 M_{\odot}$. Besides, some theoretical MSF thresholds suggest for radii smaller than $0.05 \mathrm{pc}$ a minimum core mass between 50 and $90 M_{\odot}$ (e.g. see Fig. 1 in Kauffmann \& Pillai 2010). In this context we need to precise the mass, density and size criteria to refine the number of starless MDCs able to form a high-mass star. Indeed, densities as low as $\mathrm{n}_{\mathrm{H}_{2}} \sim 10^{4}-10^{6} \mathrm{~cm}^{-3}$ is characteristic of low-mass pre-stellar cores (Könyves et al. 2015; Motte et al. 2007; Ward-Thompson et al. 1999) while small $(<0.1 \mathrm{pc})$, high mass $\left(M>100 M_{\odot}\right)$ and high density $\left(n_{\mathrm{H}_{2}}>5 \times 10^{6} \mathrm{~cm}^{-3}\right)$ are good criteria to select starless MDCs which could produce high-mass stars (e.g. Tigé et al. 2017; Urquhart et al. 2018). More generally, high resolution observations show that the dense $\left(n_{\mathrm{H}_{2}}>10^{5} \mathrm{~cm}^{-3}\right.$, compact $(\simeq 0.1 \mathrm{pc})$ and massive clumps tend to 


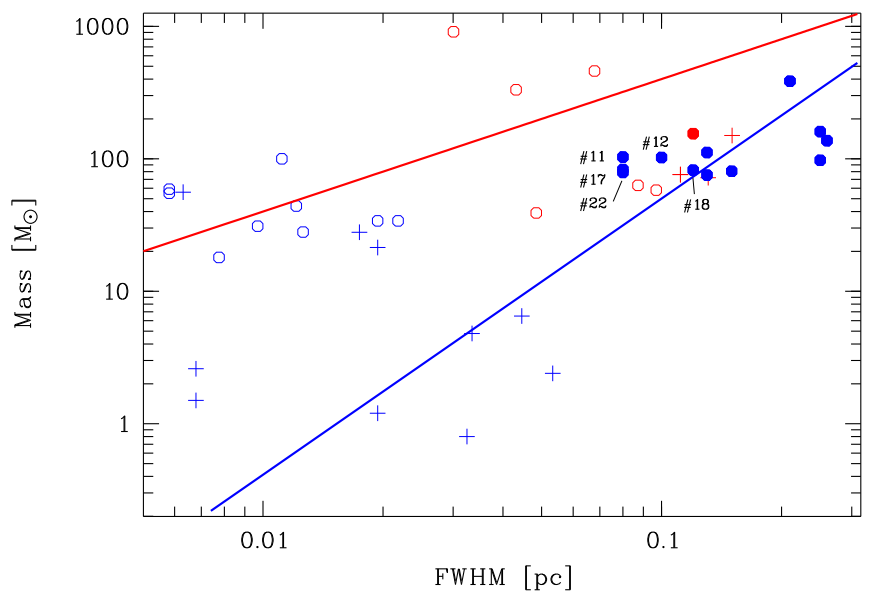

Fig. 6. Mass versus size diagram. Starless and protostellar MDC candidates in NGC 6357 are displayed as blue and red dots respectively. For comparison, following Nony et al. (2018), we also display massive starless and protostellar MDCs (Motte et al. 2007; Tigé et al. 2017; Tan et al. 2013; Louvet et al. 2014) as red crosses and circles respectively, and pre-stellar and protostellar sub-fragments and cores (Motte et al. 2018b; Tan et al. 2013; Bontemps et al. 2010; Louvet et al. 2019) as blue crosses and circles respectively. The red and blue lines represent mass radial power-laws of $\operatorname{Mass}(<r) \propto r$ and $\operatorname{Mass}(<r) \propto \mathrm{r}^{2}$.

be those that are fragmented and that contain the most massive cores (Bontemps et al. 2010; Csengeri et al. 2017b; Figueira et al. 2018; Lu et al. 2018). It is the case for example of CygX-N63 MDC (which has $n_{\mathrm{H}_{2}}=1.6410^{6} \mathrm{~cm}^{-3}$ ) and shows, at higher resolution, one massive fragment (Bontemps et al. 2010) as well the few massive protostellar cores of Lu et al. (2018) which have size $\leq 0.15 \mathrm{pc}$ and $n_{\mathrm{H}_{2}}>1.110^{6} \mathrm{~cm}^{-3}$. These characteristics are in agreement with the IR-quiet protostellar MDCs identified both in NGC 6357 and NGC 6334. For this reason, in this study, we have assumed that the compact (size $\leq 0.15 \mathrm{pc}$ ) and densest $\left(n_{\mathrm{H}_{2}}>110^{6} \mathrm{~cm}^{-3}\right)$ starless MDCs are the best candidates to form a high-mass star. At this step, the starless MDCs \#10, \#11, $\# 12$, \#17, \#18, and \#22 are the most favourable MDCs to form high-mass stars. To explore further the probability of MDCs containing high-mass starless cores or high-mass protostars we look at their mass concentration by plotting starless MDCs in the mass versus size diagram (Fig. 6). Motte et al. (2018a) and Tigé et al. (2017) recall that a mass concentration as Mass $(<r) \propto r$ is expected for $\rho \propto r^{-2}$ radial density structure (like it is observed for protostellar envelopes and the outskirt of pre-stellar cores) while Mass $(<r) \propto r^{2}$ characterise much less concentrated clouds. Tigé et al. (2017) led such analysis for NGC 6334 starless MDCs (for which $40^{\prime \prime}$ and $10^{\prime \prime}$ resolution data exist) allowing them to finally select only one best starless MDC candidate. Following the same approach, in NGC 6357 (from Fig. 6), because they stray the most from the Mass $(<r) \propto r^{2}$ law, MDCs \#10, \#11, \#12, \#17 and \#22 appear more concentrated and then more favourable to form a high-mass star. We can note that these MDCs are mainly located in features facing the clusters Pismis 24 (MDCs $\# 11$ and \#17) and AH03J1726-34.4 (MDCs \#12 and \#22).

\subsection{Relation between MDCs and filaments}

Recently Louvet et al. (2019), targeting with ALMA 13 of the 16 starless MDCs of NGC 6334, observed that four of them contain low-mass protostars while amid the others only one appears sub-structured into two low-mass pre-stellar cores. Even the best starless candidate in NGC 6334 (MDC-5) shows only low mass pre-stellar cores and protostars (Louvet et al., priv. comm.). This appears as a strong observationnal argument in agreement with the empirical model by Motte et al. (2018a) in which few lowand intermediate mass pre-stellar cores form first in MDCs and then grow in mass from the surrounding gas but some may not form high-mass stars but a low-mass cluster. Similar results for NGC 6357 starless MDCs can be suspected, but we have to understand why both regions show a similar number of starless MDCs, while they have a very different number of protostellar MDCs $(\sim 18$ and $\sim 1$ per square degree for NGC 6334 and NGC 6357, respectively). This can be linked to the filamentary structure of the regions. Indeed, Tigé et al. (2017) underline that the massive star formation in NGC 6334 is strongly related to ridge and hub features where mass can be accreted during the formation of the massive star while in NGC 6357 such features are not observed. Similarly Rayner et al. (2017) observe that in Mon R2 the massive star formation is at the centre of a filament hub.

To investigate and compare filamentary structures in NGC 6357 and NGC 6334 we ran the Vialactea Filamentary Structures Extraction Package ${ }^{19}$ on the column density maps. This package allows us to identify spatially coherent structures and determine their morphological (like lengths and geometrical shape) and physical (e.g. column density, mass) parameters. Filament spine and branches are displayed on Fig. 7. Selecting structures with length-to-width ratio larger than two (see Appendix B) we plot their properties in Fig. 8.

The histograms (Fig. 8) show that the filament properties in NGC 6334 and NGC 6357 are statistically similar, but in NGC 6334 they have parameters reaching higher values. In Fig. 8 the histogram of the deconvolved widths is presented. However, due to the distance of the regions, the filament width is not resolved at Herschel/PACS $250 \mu \mathrm{m}$ (used to produce our highresolution column density map) and are thus very uncertain. We do not use the filament width further in our analysis, their analysis is the purpose of the paper of Könyves et al. (in prep.) based on the $350 \mu \mathrm{m}$ and $8^{\prime \prime}$ resolution map ${ }^{20}$.

The filamentary difference between the two regions becomes obvious on Fig. 7. NGC 6334 is dominated by a ridge corresponding to three aligned filaments with a total mass $\sim 31807 M_{\odot}$ filament with a mass per unit length $\left(M_{\text {line }}\right)$ between 380 and $1893 M_{\odot} \mathrm{pc}^{-1}$, with a total length of $21.5 \mathrm{pc}$ and a mean width of $0.08 \mathrm{pc}$. In NGC 6357 no ridge or hub is noted, the only distinct feature is the filament (composed of three segments) around $l, b=353.5^{\circ},+0.66^{\circ}$ (hosting the IR-quiet MDC \# 6) with a mass of 585.6 $M_{\odot} \quad\left(M_{\text {line }}\right.$ between 15 and $\left.107 M_{\odot} \mathrm{pc}^{-1}\right)$, a total length of $8 \mathrm{pc}$ and a mean width of $0.16 \mathrm{pc}$. Such difference in filament and MDCs concentration is already observed at different locations of other regions as, for example, Cygnus (Motte et al. 2007), in Lupus (Rygl et al. 2013) and Perseus (Sadavoy et al. 2014).

There is increasing observational evidence that filaments with $M_{\text {line }}$ larger (thermally supercritical) than the critical value $M_{\text {line,crit }}{ }^{21}$ show evidence of pre-stellar cores and YSOs whereas thermally sub-critical filaments $\left(M_{\text {line }}<M_{\text {line,crit }}\right)$ appear

19 The Vialactea Filamentary Structures Extraction Package is available at http://vialactea.iaps.inaf.it/vialactea/eng/ tools.php

${ }^{20}$ APEX-ArtMiS, http://www . apex-telescope.org/ instruments/pi/artemis/, proposals E-094.C-0743 PI. P. André and O-094.F-9320 PI. T. Hill.

${ }^{21} M_{\text {line,crit }}$ is defined as $M_{\text {line,crit }}=2 \mathrm{c}_{s}^{2} \mathrm{G}^{-1} \sim 23$ to $42 M_{\odot} \mathrm{pc}^{-1}$ for a dust temperature of $T=14$ and $25 \mathrm{~K}$ respectively (e.g. André et al. 2010). 

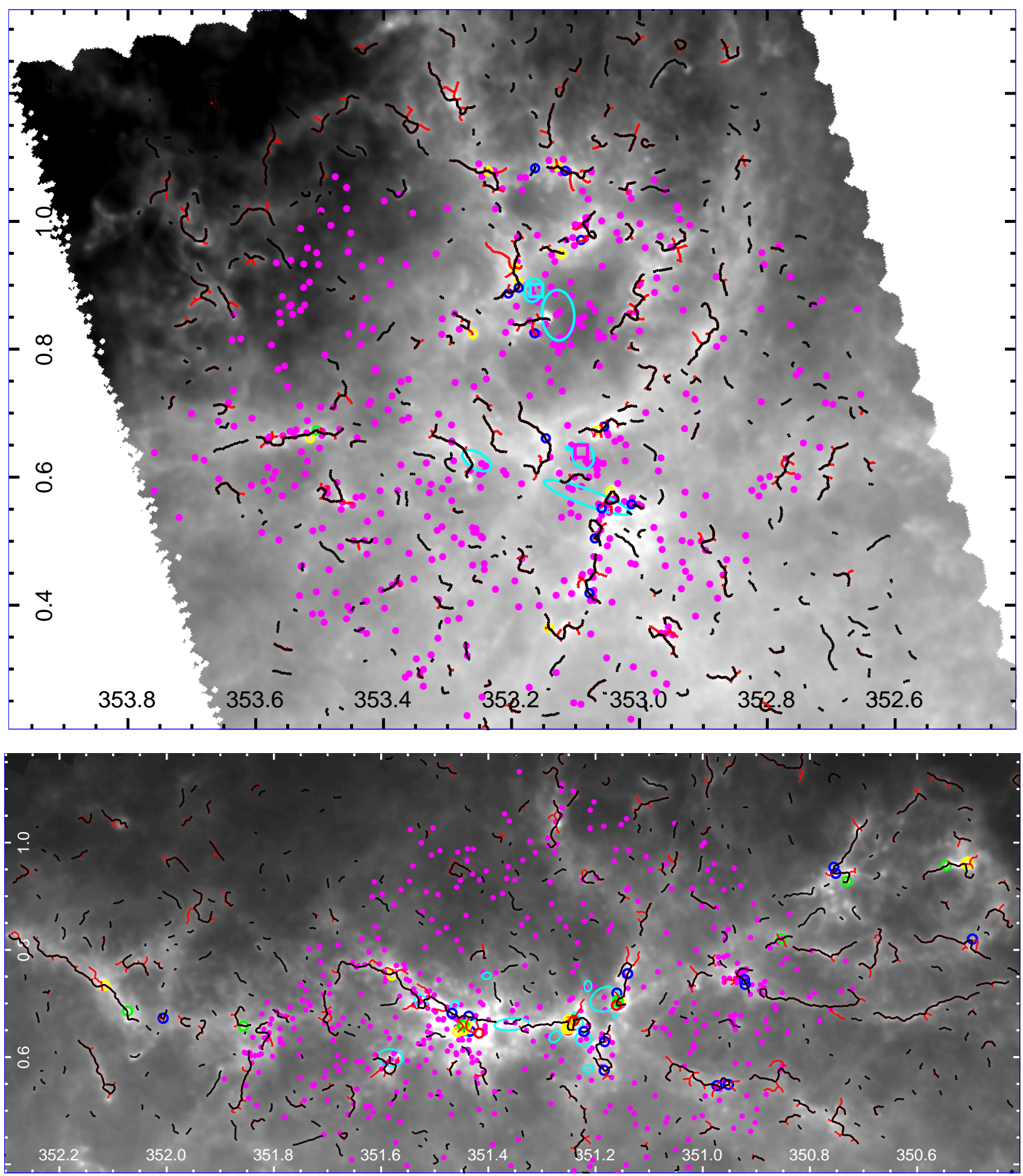

Fig. 7. NGC 6357 (upper panel) and NGC 6334 (lower panel) column density maps overlaid with class 0/I YSOs from Povich et al. (2017) (magenta dots), filaments spines (black lines) and branches (red lines) and young stellar clusters from Kuhn et al. (2015) (cyan ellipses). The MDCs colour coding is the same as in Fig. 4.

generally devoid of Herschel pre-stellar cores and protostars (André et al. 2010). This led to a proposed paradigm for solartype star formation in which low-mass stars form primarily by gravitational fragmentation of supercritical filaments (André et al. 2014). Hill et al. (2011) and Schisano et al. (2014) also suggest that the filamentary regions are more favourable to form massive stars and that MDCs are either in ridges or in hub within an $A_{\mathrm{V}}>100$ mag environment (e.g. Nguyen Luong et al. 2011b,
2013; Hill et al. 2011; Motte et al. 2018a). As highlighted by Li et al. (2016), the morphology of filaments varies from marginally resolved elongated structures to very complex networks of filaments and that they can be isolated or at the periphery of $\mathrm{H} \mathrm{II}$ regions (as they are found by Inutsuka et al. 2015 to be preferential sites of filament formation). In addition, filaments that have high linear mass density $\left(M_{\text {line }} \gg 100 M_{\odot} \mathrm{pc}^{-1}\right)$ contain large enough mass reservoirs to give birth to high-mass stars and star 

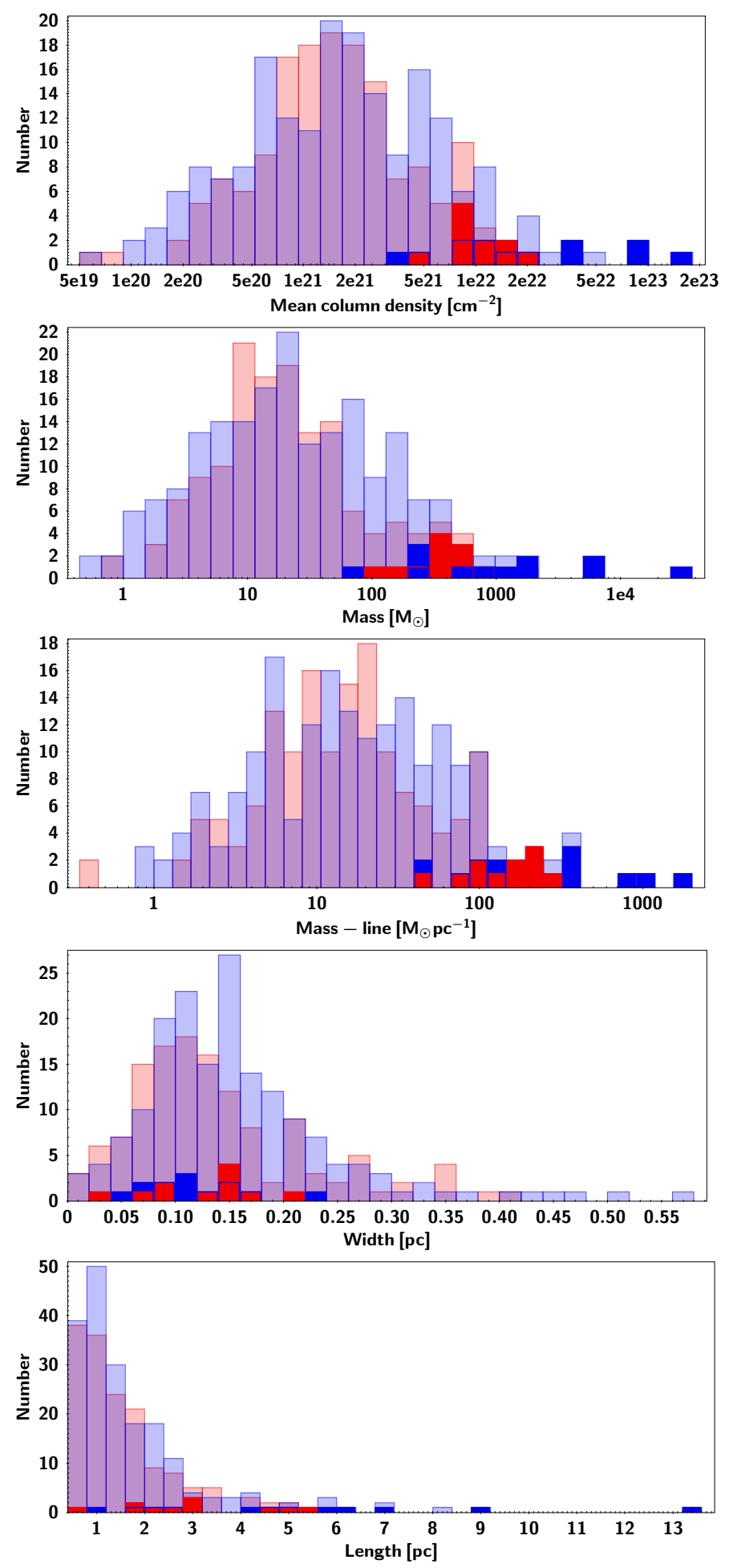

Fig. 8. Filament parameters. Light blue and red histograms correspond to NGC 6334 and NGC 6357 respectively. Filled histograms are filaments hosting MDCs. In the third panel the filament width is the deconvolved width.

clusters (e.g. Nguyen Luong et al. 2011b, 2013; Hill et al. 2011; Schneider et al. 2012; Kainulainen et al. 2013; Contreras et al. 2016; Motte et al. 2018a).

Tigé et al. (2017) and André et al. (2016) characterise the NGC 6334 ridge showing it is a very dense and massive $\left(\sim 15000 M_{\odot}\right)$ filament with a $M_{\text {line }}$ ranging from $\sim 500 M_{\odot} \mathrm{pc}^{-1}$
Table 6. Physical properties of the filaments associated to the best starless candidates.

\begin{tabular}{cccc}
\hline \hline MDC & $\begin{array}{c}\text { Fil. Mass } \\
M_{\odot}\end{array}$ & $\begin{array}{c}\text { Fil. length } \\
\text { pc }\end{array}$ & $\begin{array}{c}\text { Fil. } M_{\text {line }} \\
M_{\odot} \mathrm{pc}^{-1}\end{array}$ \\
\hline 10 & 424 & 2.3 & 179 \\
$11 \& 17$ & 507 & 4.7 & 108 \\
12 & 129 & 0.6 & 211 \\
22 & 407 & 1.8 & 220 \\
\hline
\end{tabular}

to $2000 M_{\odot} \mathrm{pc}^{-1}$ over nearly $10 \mathrm{pc}$ and a width of $0.15 \mathrm{pc}$. A gap is noted around the middle of the NGC 6334 filament probably created by the $\mathrm{H}$ II region seen in $\mathrm{H} \alpha$ at this position and illustrating how recent massive star formation disrupts filaments. In addition, Zernickel et al. (2013) observe gas flow along the ridge which can be interpreted as another hint for the model by Motte et al. (2018a), model in which MDCs grow in mass from the surrounding gas.

We clearly note that filaments more massive than $1000 M_{\odot}$ belong to NGC 6334 while the most massive filament in NGC 6357 reaches only $766 M_{\odot}$. In both regions, MDCs are located on the filament, spine, or branch and they belong to the most massive and highest $M_{\text {line }}$ filaments (Fig. 8) indicating that filaments are important structures for the formation of massive stars. Among the five best candidates the MDCs \#11 and \#17 are located on a spine; the others are either on a branch or at the end of a small spine. Therefore, if we assumed that mass feeding along filaments is an effective process, MDC \#17 could be the best to grow in mass as it is located at a branch to spine junction.

We can use the mass and the $M_{\text {line }}$ to estimate which of the starless MDCs in NGC 6357 has enough mass reservoir to form a massive star. The IR-quiet MDC (MDC \# 6) belongs to a $5 \mathrm{pc}$ long, $537 M_{\odot}, M_{\text {line }}=107 M_{\odot} \mathrm{pc}^{-1}$ segment (while in NGC 6334 IR-quiet MDCs belong to filaments with $M_{\text {line }}$ between $224 M_{\odot} \mathrm{pc}^{-1}$ and $1152 M_{\odot} \mathrm{pc}^{-1}$ ). In this way, the starless MDCs \#10, \#11, \#12, \#17 and \#22 are all in filaments that have $M_{\text {line }}>107 M_{\odot} \mathrm{pc}^{-1}$ (see Table 6). MDC \#12 is quite peculiar because it is located at the end of a short $(0.61 \mathrm{pc})$ filament (clump-like filament). Its velocity analysis (Sect. 4.3) indicates infall but the filament mass is similar to its mass suggesting that either the mass reservoir is not enough to form a massive star or that it is in a slightly more evolved stage than the other starless MDCs.

We also use class 0/I YSOs (from Povich et al. 2017) to probe the star formation activity of filaments where the starless MDCs are. Indeed, Rivilla et al. $(2013,2014)$ show that YSOs and low mass pre-main sequence stars tend to be clustered at the massive star-forming sites. They interpret that as evidence of the "competitive theory" for massive star-formation (e.g. Bonnell \& Bate 2006), where a low-mass stellar cluster creates a potential well which funnels gas and dust towards its centre where the most massive stars will form. In this frame, we can speculate that MDCs not surrounded by YSOs could be less evolved or have less probability to form a massive star.

Several YSOs are identified around the MDCs \#10, \#12, and \#22. Especially, around MDC\#22 their spatial distribution seems to delineate an elongated structure (from $l, b=353.04^{\circ}$, $+0.57^{\circ}$ to $\left.l, b=353.07^{\circ},+0.40^{\circ}\right)$ which encompasses three filaments (Fig. A.1). In this picture the parental filament would be $7.4 \mathrm{pc}$ long, with Mass $=1188 M_{\odot}$ and $M_{\text {line }}=161 M_{\odot} \mathrm{pc}^{-1}$. Towards it we note four WISE detected compact H II regions 
Table 7. Median properties for starless candidates MDCs in NGC 6334 and NGC 6357.

\begin{tabular}{|c|c|c|}
\hline & $\begin{array}{c}\text { NGC } 6334 \\
\text { Tigé et al. (2017) }\end{array}$ & NGC 6357 \\
\hline Number & 16 & 12 \\
\hline Size $(p c)$ & 0.14 & 0.13 \\
\hline$\left\langle T_{\text {dust }}\right\rangle(\mathrm{K})$ & 15 & 17 \\
\hline$L_{\mathrm{bol}}\left(L_{\odot}\right)$ & 130 & $431^{(b)}$ \\
\hline $\operatorname{Mass}\left(M_{\odot}\right)$ & 103 & 102 \\
\hline$\left\langle n_{\mathrm{H}_{2}}\right\rangle\left(\mathrm{cm}^{-3}\right)$ & $1.4 \times 10^{6}$ & $1.3 \times 10^{6}$ \\
\hline Free-fall time ${ }^{(a)}(\mathrm{yr})$ & $3 \times 10^{4}$ & $3.2 \times 10^{4}$ \\
\hline
\end{tabular}

Notes. ${ }^{(a)}$ Free-fall time $\left(t_{\mathrm{ff}}\right)$ measured from the median values of the density averaged over the full MDC volume, which is approximately a sphere with a FWHM radius: $\left\langle n_{\mathrm{H}_{2}}\right\rangle_{\text {full }}=\left\langle n_{\mathrm{H}_{2}}\right\rangle / 8$ and $t_{\text {free-fall }}=\sqrt{\frac{3 \pi}{32 G \mu m_{\mathrm{H}}\left\langle n_{\mathrm{H}_{2}}\right\rangle \text { full }}} \cdot{ }^{(b)}$ Due to the large uncertainty on $L_{\mathrm{bol}}$ and any possible NIR source fortuitous association this value can reach a lower limit of $319 L_{\odot}$ (considering only that $L_{\mathrm{bol}}=L_{\mathrm{Fit}}$ ).

(Anderson et al. 2014) suggesting that massive star-formation is already processing at this place, disrupting the filament. In this context, we can suspect that MDC\#22 would not have enough mass to accrete to form a high-mass star. MDC\#10 is in a branch (Fig. A.1) of a thick filament which has started at least low mass star-formation at its other spine-to-branch junction. It is difficult to predict if MDC\#10 will be able to grow in mass because it is in a quite isolated area. Towards the filament where MDC\#17 and MDC\#11 sit, no YSOs are noted. MDC\#17, however, being located at a branch to spine junction, appears then as the most favourable starless MDC to form a high-mass star. In addition, on the $350 \mu \mathrm{m}$ and $8^{\prime \prime}$ resolution map (Könyves et al., in prep.), only MDC\#11 and MDC\#17 are centrally peaked sources, additionnally suggesting they are the most favourable MDCs to form high-mass star.

\subsection{NGC 6357 and NGC 6334 history}

Comparing MDCs for both regions (see Table 7) we can note that they have similar properties except that $L_{\text {bol }}$ is about seven times larger for starless MDCs in NGC 6357. Following Ward-Thompson et al. (2002) this higher $L_{\mathrm{bol}}$ reflects that the external heating of the starless cores by the local radiation field is higher in NGC 6357 than in NGC 6334. This is expected as NGC 6357 is powered by a the rich OB star cluster Pismis 24 while no such strong radiation source is noted in NGC 6334.

In addition, from his sample of protostellar MDCs, Tigé et al. (2017) estimate a statistical lifetime of $3.5 \times 10^{5} \mathrm{yr}$. Such statistical lifetime is estimated from the relative number of a given MDC phase to the OB stars. For NGC 6357 we estimate a total number of 60 O-B3 stars from Russeil et al. (2012, 2017) and Povich et al. (2017). We assume a median age of the O-B3 stars to be $1 \times 10^{6} \mathrm{yr}$ according to Fang et al. (2012) and Getman et al. (2014). To evaluate the typical lifetime in NGC 6357 we have to estimate the number of massive stars our MDCs can form. Then, following Tigé et al. (2017), and in agreement with Csengeri et al. (2017a), we adopt the same fragmentation level found in Cygnus $\mathrm{X}$ protostellar MDCs by Bontemps et al. (2010) to assume that IR-quiet MDCs should host on average two high-mass stars. For high-mass protostellar cores this gives us a statistical lifetime of $3.3 \times 10^{4} \mathrm{yr}$ corresponding to the free-fall time. This unrealistically small value suggests that the massive star formation has stopped for at least the last Myr and that NGC 6357 will not form any more massive star.

From the Miville-Deschênes et al. (2017) molecular cloud catalogue, based on ${ }^{12} \mathrm{CO}$ emission, we estimate a total gas mass of $1.6 \times 10^{5} M_{\odot}$ (this can be compared with the mass estimation of $2.3 \times 10^{5} M_{\odot}$ from Willis et al. 2013 and Schneider et al. 2015) and $2.4 \times 10^{5} M_{\odot}$ (which can be compared with the mass estimation of $4 \times 10^{5}$ from Cappa et al. 2011) for NGC 6334 and NGC 6357 respectively. The total filament masses are $\sim 5.4 \times 10^{4} M_{\odot}$ and $1.02 \times 10^{4} M_{\odot}$ giving that about 25 and $4 \%$ of the mass is in the form of filaments in NGC 6334 and NGC 6357, respectively. Considering the total MDC mass, we estimate that about 9 and $14 \%$ of the filament mass is in the form of MDCs, for both regions respectively. Comparatively, the estimated total massive core formation efficienty (TCFE) is about 3 and $0.7 \%$ for NGC 6334 and NGC 6357, respectively.

The young stellar population also appears very different in the two regions. While the young stellar clusters, identified by Kuhn et al. (2015), are mainly located along the NGC 6334's ridge and hub, in NGC 6357 they are either associated to the clusters Pismis 24 or AH03J1725-34.4. In parallel, Getman et al. (2014) underline an age gradient (between 2.3 and 0.7 Myr and from the south-west to the north-east) of the young stellar cluster along the NGC 6334's filaments while this is not the case in NGC 6357 (clusters and the uniformly distributed stellar population have similar ages between 1.0 and $1.5 \mathrm{Myr}$ ), suggesting that the recent star formation proceeded nearly simultaneously across NGC 6357. However, because the Wolf-Rayet phase is expected to occur at a stellar age of 3 Myr (e.g. Sokal et al. 2016) and because OB stars with ages around 4.6 Myr were also reported towards NGC 6357 (Russeil et al. 2017), we can suspect that starformation in NGC 6357 has been active for at least 5 Myr. The large (radius $15 \mathrm{pc}$ ) $\mathrm{H} \alpha$ ring (as defined by Massi et al. 2015) could even have been shaped by a previous event as Russeil et al. (2017) note shock-heated gas towards the filaments on its northeast side. Adopting an expansion velocity of $5 \mathrm{~km} \mathrm{~s}^{-1}$ would give and age of 3.6 Myr for this ring, which could make it a relic of a previous massive star formation episode.

So we can speculate that previous feedback and the present feedback from O-type stars in Pismis 24 (e.g. Massey et al. 2001 list in Pismis-24 at least two O3 and one WR stars), have stopped the star formation by dispersing its molecular cloud. This is in agreement with the results of Walch et al. (2012) who show that a single $\mathrm{O} 7$ star is able to photo-ionise and disperse a $10^{4} M_{\odot}$ molecular cloud in 1-2 Myr. NGC 6334 harbouring fewer and less-massive stars (e.g. Persi \& Tapia 2008) the gas removal timescale would then be longer. In addition, NGC 6357 follows qualitatively the evolutionnary picture of the star formation (at kpc scale) with EUV and SN feedback as simulated by Butler et al. (2017). They show that feedback tends to disperse the clustering of the star-formation and to reduce the star formation rate (especially when the mechanical feedback from radiation and supernovae is combined).

On a larger scale, a 100-pc scale feature can be underlined by connecting the young stellar clusters located in NGC 6334 and NGC 6357 (Fang et al. 2012; Kuhn et al. 2014, 2015; Massi et al. 2015), the ridge in NGC 6334, the filament (at $l, b=353.5^{\circ},+0.66^{\circ}$ ) in NGC 6357, the filament (seen in on extinction map) connecting NGC 6334 and NGC 6357 (Russeil et al. 2010) and the G350.54+0.69 filament (Liu et al. 2018). They trace a 100-pc long feature aligned with the Galactic plane at $b \sim 0.67^{\circ}(20 \mathrm{pc}$ above the Galactic plane at a distance of $1.75 \mathrm{kpc}$ ) which could trace the parental filament of both 
star-forming regions. In this scheme, following Fukui et al. (2018) the formation of NGC 6357 and NGC 6334 could have been triggered by a $100 \mathrm{pc}$-scale cloud-cloud collision.

\section{Conclusions}

In the framework of the Herschel/HOBYS key programme and in the same way as is done for NGC 6334 by Tigé et al. (2017), we performed a study of the massive dense cores in NGC 6357 to better understand how high-mass stars form. We combined the Herschel/HOBYS images to mid-infrared and (sub-)millimeter ground-based data to obtain a complete census of 23 MDCs, among which five are expected to be the most probable progenitors of high-mass stars at $0.1 \mathrm{pc}$ scale. These starless MDCs belong mainly to the edge of the Pismis-24 cavity and the region $\mathrm{H}$ II 353.09+0.63 (excited by AH03J1726-34.4). We confirm that, contrarily to NGC 6334, no ridge and hub which can feed the MDCs are observed in NGC 6357. Filaments in NGC 6334 reach higher mass and higher $M_{\text {line }}$ than in NGC 6357. In both regions MDCs sit in the most massive filaments underlying their importance for the formation of massive stars in agreement with the empirical model by Motte et al. (2018a) in which MDCs and protostars grow in mass from the surrounding gas. In this picture, because the hottest stars in Pismis-24 have disrupted filaments, the massive star formation in NGC 6357 seems to have stopped and we speculate that little massive star formation will occur.

Acknowledgement. SPIRE has been developed by a consortium of institutes led by Cardiff University (UK) and including University of Lethbridge (Canada) NAOC (China); CEA, LAM (France); IFSI, University of Padua (Italy); IAC (Spain); Stockholm Observatory (Sweden); Imperial College London, RAL, UCL-MSSL, UKATC, University of Sussex (UK); and Caltech, JPL, NHSC, University of Colorado (USA). This development has been supported by national funding agencies: CSA (Canada); NAOC (China); CEA, CNES, CNRS (France); ASI (Italy); MCINN (Spain); SNSB (Sweden); STFC, UKSA (UK); and NASA (USA). PACS has been developed by a consortium of institutes led by MPE (Germany) and including UVIE (Austria); KU Leuven, CSL, IMEC (Belgium); CEA, LAM (France); MPIA (Germany); INAF-IFSI/OAA/OAP/OAT, LENS, SISSA (Italy); IAC (Spain). This development has been supported by the funding agencies BMVIT (Austria), ESA-PRODEX (Belgium), CEA/CNES (France), DLR (Germany), ASI/INAF (Italy), and CICYT/MCYT (Spain). This research has made use of the SIMBAD database, operated at CDS, Strasbourg, France. K.L.J.R. acknowledges financial support by the Italian Ministero dell'Istruzione Università e Ricerca through the grant Progetti Premiali 2012-iALMA (CUP C52I13000140001). N.S. and S.B. acknowledge support by the french ANR and the german DFG through the project "GENESIS" (ANR-16-CE92-003501/DFG1591/2-1). GLW gratefully acknowledges support from The Leverhulme Trust. We acknowledge financial support from "Programme National de Physique Stellaire" (PNPS) and programme "Physique et Chime du Milieu Interstellaire" (PCMI) of CNRS/INSU, France. A.Z. thanks the support of the Institut Universitaire de France.

\section{References}

Alves, J., Lombardi, M., \& Lada, C. J. 2007, A\&A, 462, L17 Anderson, L. D., Bania, T. M., Balser, D. S., et al. 2014, ApJS, 212, 1 André, P., Men'shchikov, A., Bontemps, S., et al. 2010, A\&A, 518, L102 André, P., Di Francesco, J., Ward-Thompson, D., et al. 2014, Protostars and Planets VI (Tucson: University of Arizona Press), 27

André, P., Revéret, V., Könyves, V., et al. 2016, A\&A, 592, A54

Baldeschi, A., Elia, D., Molinari, S., et al. 2017, MNRAS, 466, 3682

Benjamin, R. A., Churchwell, E., Babler, B. L., et al. 2003, PASP, 115, 953

Bertoldi, F., \& McKee, C. F. 1992, ApJ, 395, 140

Beuther, H., Schilke, P., Menten, K. M., et al. 2002, ApJ, 566, 945

Bonnell, I. A., \& Bate, M. R. 2006, MNRAS, 370, 488

Bontemps, S., Motte, F., Csengeri, T., \& Schneider, N. 2010, A\&A, 524, A18

Butler, M. J., Tan, J. C., Teyssier, R., et al. 2017, ApJ, 841, 82

Cappa, C. E., Barbá, R., Duronea, N. U., et al. 2011, MNRAS, 415, 2844
Carey, S. J., Noriega-Crespo, A., Mizuno, D. R., et al. 2009, PASP, 121, 76 Caswell, J. L., \& Haynes, R. F. 1987, A\&A, 171, 261

Caswell, J. L., Fuller, G. A., Green, J. A., et al. 2010, MNRAS, 404, 1029 Chambers, E. T., Yusef-Zadeh, F., \& Ott, J. 2014, A\&A, 563, A68 Condon, J. J., Cotton, W. D., Greisen, E. W., et al. 1998, AJ, 115, 1693

Contreras, Y., Garay, G., Rathborne, J. M., \& Sanhueza, P. 2016, MNRAS, 456, 2041

Csengeri, T., Bontemps, S., Schneider, N., Motte, F., \& Dib, S. 2011, A\&A, 527, A 135

Csengeri, T., Urquhart, J. S., Schuller, F., et al. 2014, A\&A, 565, A75

Csengeri, T., Bontemps, S., Wyrowski, F., et al. 2017a, A\&A, 600, L10

Csengeri, T., Bontemps, S., Wyrowski, F., et al. 2017b, A\&A, 601, A60 Cyganowski, C. J., Whitney, B. A., Holden, E., et al. 2008, AJ, 136, 2391 Dias, W. S., Alessi, B. S., Moitinho, A., \& Lépine, J. R. D. 2002, A\&A, 389, 871

Duarte-Cabral, A., Bontemps, S., Motte, F., et al. 2013, A\&A, 558, A125

Egan, M. P., Price, S. D., \& Kraemer, K. E. 2003, BAAS, 35, 1301

Fang, M., van Boekel, R., King, R. R., et al. 2012, A\&A, 539, A119

Figueira, M., Bronfman, L., Zavagno, A., et al. 2018, A\&A, 616, L10

Foster, J. B., Rathborne, J. M., Sanhueza, P., et al. 2013, PASA, 30, e038

Fukui, Y., Kohno, M., Yokoyama, K., et al. 2018, PASJ, 70, S41

Fuller, G. A., Williams, S. J., \& Sridharan, T. K. 2005, A\&A, 442, 949

Getman, K. V., Feigelson, E. D., Kuhn, M. A., et al. 2014, ApJ, 787, 108

Giannetti, A., Brand, J., Massi, F., Tieftrunk, A., \& Beltrán, M. T. 2012, A\&A, 538, A41

Giannetti, A., Leurini, S., Wyrowski, F., et al. 2017, A\&A, 603, A33

Ginsburg, A., \& Mirocha, J. 2011, Astrophysics Source Code Library [record ascl: 1109.001]

Giveon, U., Becker, R. H., Helfand, D. J., \& White, R. L. 2005, AJ, 129, 348

Griffin, M. J., Abergel, A., Abreu, A., et al. 2010, A\&A, 518, L3

He, Y.-X., Zhou, J.-J., Esimbek, J., et al. 2015, MNRAS, 450, 1926

Hildebrand, R. H. 1983, QJRAS, 24, 267

Hill, T., Motte, F., Didelon, P., et al. 2011, A\&A, 533, A94

Hill, T., Motte, F., Didelon, P., et al. 2012, A\&A, 542, A114

Inutsuka, S.-i., Inoue, T., Iwasaki, K., \& Hosokawa, T. 2015, A\&A, 580, A49

Jackson, J. M., Rathborne, J. M., Foster, J. B., et al. 2013, PASA, 30, e057

Jenness, T., Currie, M. J., Tilanus, R. P. J., et al. 2015, MNRAS, 453, 73

Kainulainen, J., Ragan, S. E., Henning, T., \& Stutz, A. 2013, A\&A, 557, A120

Kauffmann, J., \& Pillai, T. 2010, ApJ, 723, L7

König, C., Urquhart, J. S., Csengeri, T., et al. 2017, A\&A, 599, A139

Könyves, V., André, P., Men'shchikov, A., et al. 2015, A\&A, 584, A91

Kuhn, M. A., Feigelson, E. D., Getman, K. V., et al. 2014, ApJ, 787, 107

Kuhn, M. A., Getman, K. V., \& Feigelson, E. D. 2015, ApJ, 802, 60

Li, G.-X., Urquhart, J. S., Leurini, S., et al. 2016, A\&A, 591, A5

Liu, H.-L., Stutz, A., \& Yuan, J.-H. 2018, MNRAS, 478, 2119

Louvet, F., Motte, F., Hennebelle, P., et al. 2014, A\&A, 570, A15

Louvet, F., Neupane, S., Garay, G., et al. 2019, A\&A, 622, A99

Lu, X., Zhang, Q., Liu, H. B., et al. 2018, ApJ, 855, 9

Mardones, D., Myers, P. C., Tafalla, M., et al. 1997, ApJ, 489, 719

Massey, P., DeGioia-Eastwood, K., \& Waterhouse, E. 2001, AJ, 121, 1050

Massi, F., Brand, J., \& Felli, M. 1997, A\&A, 320, 972

Massi, F., Giannetti, A., Di Carlo, E., et al. 2015, A\&A, 573, A95

Matthews, H. E., McCutcheon, W. H., Kirk, H., White, G. J., \& Cohen, M. 2008 AJ, 136, 2083

Men'shchikov, A. 2013, A\&A, 560, A63

Men'shchikov, A. 2016, A\&A, 593, A71

Men'shchikov, A. 2017, A\&A, 607, A64

Men'shchikov, A., André, P., Didelon, P., et al. 2012, A\&A, 542, A81

Miville-Deschênes, M.-A., Murray, N., \& Lee, E. J. 2017, ApJ, 834, 57

Molinari, S., Pezzuto, S., Cesaroni, R., et al. 2008, A\&A, 481, 345

Molinari, S., Schisano, E., Elia, D., et al. 2016, A\&A, 591, A149

Motte, F., Bontemps, S., Schilke, P., et al. 2007, A\&A, 476, 1243

Motte, F., Zavagno, A., Bontemps, S., et al. 2010, A\&A, 518, L77

Motte, F., Bontemps, S., \& Louvet, F. 2018a, ARA\&A, 56, 41

Motte, F., Nony, T., Louvet, F., et al. 2018b, Nat. Astron., 2, 478

Muñoz, D. J., Mardones, D., Garay, G., et al. 2007, ApJ, 668, 906

Nguyen Luong, Q., Motte, F., Hennemann, M., et al. 2011a, A\&A, 535, A76

Nguyen Luong, Q., Motte, F., Schuller, F., et al. 2011b, A\&A, 529, A41

Nguyen-Luong, Q., Motte, F., Carlhoff, P., et al. 2013, ApJ, 775, 88

Nony, T., Louvet, F., Motte, F., et al. 2018, A\&A, 618, L5

Ott, S. 2010, in Astronomical Data Analysis Software and Systems XIX, eds.

Y. Mizumoto, K.-I. Morita, \& M. Ohishi, ASP Conf. Ser., 434, 139

Palmeirim, P., André, P., Kirk, J., et al. 2013, A\&A, 550, A38

Peretto, N., Fuller, G. A., André, P., et al. 2014, A\&A, 561, A83

Persi, P., \& Tapia, M. 2008, Star Formation in NGC 6334 (San Francisco: ASP), ed. B. Reipurth, 456

Poglitsch, A., Waelkens, C., Geis, N., et al. 2010, A\&A, 518, L2 
Povich, M. S., Busk, H. A., Feigelson, E. D., Townsley, L. K., \& Kuhn, M. A. 2017, ApJ, 838, 61

Rayner, T. S. M., Griffin, M. J., Schneider, N., et al. 2017, A\&A, 607, A22

Rivilla, V. M., Martín-Pintado, J., Jiménez-Serra, I., \& Rodríguez-Franco, A. 2013, A\&A, 554, A48

Rivilla, V. M., Jiménez-Serra, I., Martín-Pintado, J., \& Sanz-Forcada, J. 2014, MNRAS, 437, 1561

Roussel, H. 2013, PASP, 125, 1126

Russeil, D., Zavagno, A., Motte, F., et al. 2010, A\&A, 515, A55

Russeil, D., Zavagno, A., Adami, C., et al. 2012, A\&A, 538, A142

Russeil, D., Tigé, J., Adami, C., et al. 2016, A\&A, 587, A135

Russeil, D., Adami, C., Bouret, J. C., et al. 2017, A\&A, 607, A86

Rygl, K. L. J., Benedettini, M., Schisano, E., et al. 2013, A\&A, 549, L1

Sadavoy, S. I., Di Francesco, J., André, P., et al. 2014, ApJ, 787, L18

Schisano, E., Rygl, K. L. J., Molinari, S., et al. 2014, ApJ, 791, 27

Schneider, N., Csengeri, T., Bontemps, S., et al. 2010, A\&A, 520, A49

Schneider, N., Csengeri, T., Hennemann, M., et al. 2012, A\&A, 540, L11

Schneider, N., Bontemps, S., Girichidis, P., et al. 2015, MNRAS, 453, 41

Schuller, F., Menten, K. M., Contreras, Y., et al. 2009, A\&A, 504, 415
Sokal, K. R., Johnson, K. E., Indebetouw, R., \& Massey, P. 2016, ApJ, 826, 194

Tan, J. C., Kong, S., Butler, M. J., Caselli, P., \& Fontani, F. 2013, ApJ, 779, 96

Tigé, J. 2014, Ph.D. Thesis, Laboratoire d'Astrophysique de Marseille (LAM), Université Aix-Marseille, France

Tigé, J., Motte, F., Russeil, D., et al. 2017, A\&A, 602, A77

Urquhart, J. S., Moore, T. J. T., Schuller, F., et al. 2013, MNRAS, 431, 1752

Urquhart, J. S., Csengeri, T., Wyrowski, F., et al. 2014, A\&A, 568, A41

Urquhart, J. S., König, C., Giannetti, A., et al. 2018, MNRAS, 473, 1059

Walch, S. K., Whitworth, A. P., Bisbas, T., Wünsch, R., \& Hubber, D. 2012, MNRAS, 427, 625

Ward-Thompson, D., Motte, F., \& Andre, P. 1999, MNRAS, 305, 143

Ward-Thompson, D., André, P., \& Kirk, J. M. 2002, MNRAS, 329, 257

White, R. L., Becker, R. H., \& Helfand, D. J. 2005, AJ, 130, 586

Whitney, B. A., Indebetouw, R., Bjorkman, J. E., \& Wood, K. 2004, ApJ, 617, 1177

Willis, S., Marengo, M., Allen, L., et al. 2013, ApJ, 778, 96

Wright, E. L., Eisenhardt, P. R. M., Mainzer, A. K., et al. 2010, AJ, 140, 1868

Zernickel, A., Schilke, P., \& Smith, R. J. 2013, A\&A, 554, L2 


\section{Appendix A: Additional figure}
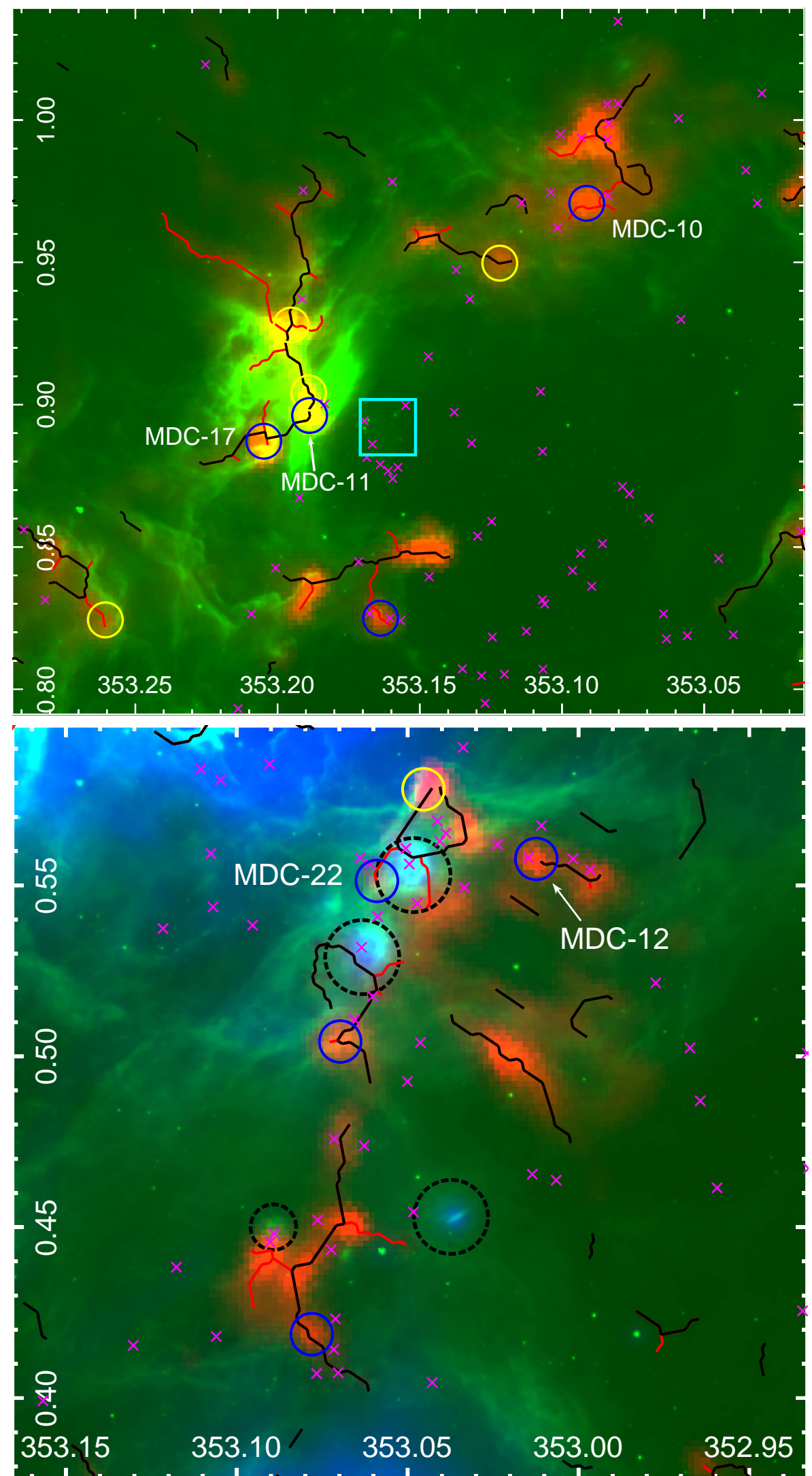

Fig. A.1. Colour views (with Galactic 1 and b coordinates) of the MDCs \#10, \#11, \#12, \#17, and \#22 (symbols are the same as in Fig. 4): WISE- $22 \mu \mathrm{m}$ (blue), column density (red) and Spitzer- $8 \mu \mathrm{m}$ (green). For the upper panel the WISE- $22 \mu \mathrm{m}$ emission is not displayed because it is saturated. The black dashed circles are the WISE detected compact H II regions from Anderson et al. (2014). Magenta symbols are class 0/I YSOs from Povich et al. (2017). 


\section{Appendix B: NGC 6357 filament validation}

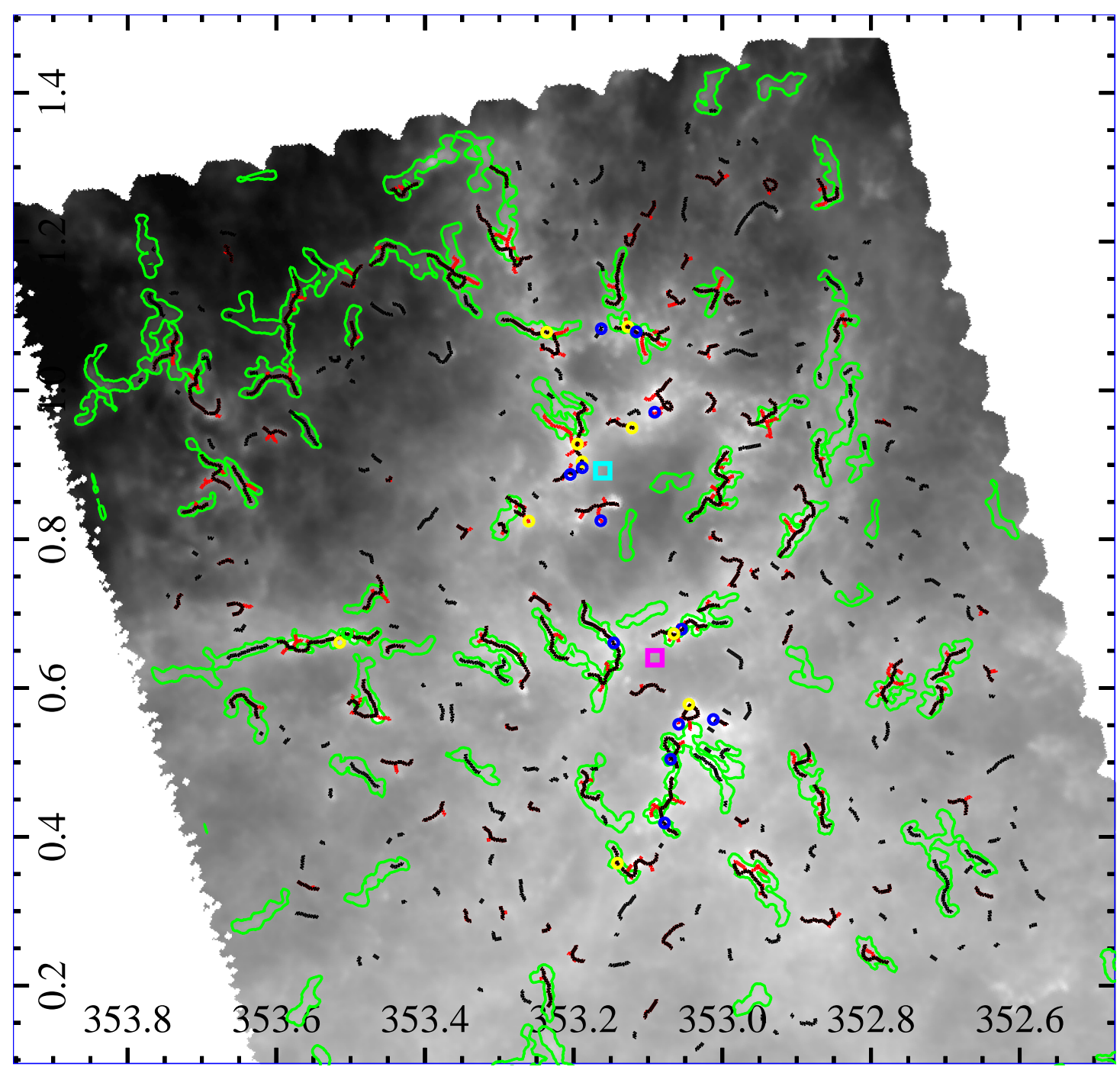

Fig. B.1. Column density map (symbols are the same as in Fig. 4, coordinates are Galactic 1,b) overlaid with the spines (black lines) and branches (red lines) defined by the Vialactea Filamentary Structures Extraction Package (with a threshold of 4) and with the filament isocontours (in green) from getfilaments (up to a spatial scale of $72^{\prime \prime}$ ).

In addition to extracting filaments with the Vialactea Filamentary Structures Extraction Package (hereafter VFSEP, Schisano et al. 2014 and in prep.) we also performed the extraction using getfilaments a multi-scale, multi-wavelength filament extraction method (Men'shchikov 2013). getfilaments analyses decompositions of original image (here the column density map) across a wide range of spatial scales, the latter being separated by a small amount (a factor of 1.05) while the Vialactea Filamentary Structures Extraction Package defines a filament as a two-dimensional elongated region with a relatively higher brightness contrast with respect to its surroundings, and uses a differential method (investigating the eigenvalues of the Hessian matrix of the intensity field) directly related to the contrast.

We note that the two methods are in overall agreement and that VFSEP resolved small marginally elongated structures. All of the MDCs are within a VFSEP filament while about half of them do not belong a filament when using getfilaments. In the paper we have used the VFSEP filaments but Schisano et al. (2014) indicate that relatively roundish structures like large and elongated compact clumps, or clusters of compact objects lying on a strong intensity field, might also be detected. To discard such "roundish" structures, we then consider only filaments with length to width ratio larger than 2 . 


\section{Appendix C: Catalogues}

Table C.1. Physical properties of the 155 robust sources.

\begin{tabular}{ccccccccc}
\hline \hline $\begin{array}{c}\text { Source } \\
\text { nb. }\end{array}$ & $\begin{array}{c}F W H M_{\text {dec }} \\
(\mathrm{pc})\end{array}$ & $\begin{array}{c}\left\langle T_{\text {dust }}\right\rangle \\
(\mathrm{K})\end{array}$ & $\begin{array}{c}\text { Mass } \\
\left(M_{\odot}\right)\end{array}$ & $\begin{array}{c}L_{\text {Data }} \\
\left(L_{\odot}\right)\end{array}$ & $\begin{array}{c}L_{\mathrm{Fit}} \\
\left(L_{\odot}\right)\end{array}$ & $\begin{array}{c}\left\langle n_{\mathrm{H}_{2}}\right\rangle \\
\left(\times 10^{6} \mathrm{~cm}^{-3}\right)\end{array}$ & $\begin{array}{c}\mathrm{RA}_{2000}, \mathrm{Dec}_{2000} \\
\left(\mathrm{~h} \mathrm{~m} \mathrm{~s},{ }^{\circ}{ }^{\prime \prime}\right)\end{array}$ & $\begin{array}{c}\text { MDC } \\
\text { nb. }\end{array}$ \\
\hline 1 & 0.05 & $27.7 \pm 0.4$ & $32.4 \pm 2.4$ & 2275 & 1388 & $7.32 \pm 0.54$ & $17: 26: 51.60,-34: 08: 25.14$ \\
2 & 0.05 & $32.0 \pm 0.7$ & $2.1 \pm 0.2$ & 352 & 216 & $0.47 \pm 0.06$ & $17: 25: 26.52,-34: 38: 12.28$ & \\
3 & 0.05 & $31.2 \pm 2.3$ & $48.7 \pm 8.6$ & 6900 & 4309 & $11.01 \pm 1.95$ & $17: 26: 01.61,-34: 15: 14.72$ \\
4 & 0.05 & $34.7 \pm 0.9$ & $1.1 \pm 0.1$ & 279 & 177 & $0.24 \pm 0.03$ & $17: 28: 03.26,-34: 18: 28.77$ \\
5 & 0.05 & $30.8 \pm 0.6$ & $4.6 \pm 0.5$ & 675 & 372 & $1.03 \pm 0.1$ & $17: 27: 10.27,-34: 16: 39.86$ \\
6 & 0.05 & $19.1 \pm 0.9$ & $27.3 \pm 5.1$ & 186 & 127 & $6.16 \pm 1.16$ & $17: 26: 46.96,-33: 59: 24.13$ \\
7 & 0.05 & $18.7 \pm 1.5$ & $5.5 \pm 1.8$ & 131 & 23 & $1.24 \pm 0.41$ & $17: 24: 41.98,-34: 40: 37.63$ \\
8 & 0.05 & $18.6 \pm 1.2$ & $16.2 \pm 4.5$ & 146 & 64 & $3.67 \pm 1.02$ & $17: 26: 45.90,-33: 59: 27.97$ \\
9 & 0.07 & $28.0 \pm 2.5$ & $2.8 \pm 0.7$ & 251 & 127 & $0.22 \pm 0.06$ & $17: 27: 46.85,-34: 16: 55.39$ \\
10 & 0.05 & $15.9 \pm 0.8$ & $17.4 \pm 4.3$ & 47 & 27 & $3.27 \pm 0.8$ & $17: 26: 21.50,-34: 47: 02.20$ \\
\hline
\end{tabular}

Notes. The full table is available at the CDS.

The 155 selected robust sources are presented in Table C.1 (only the ten first sources are displayed, the full table is available at the CDS). In addition, the NGC 6357 HOBYS catalogue tables for the 23 MDCs are given here (ordered by mass). Table C.2 gives the source position and the Herschel/PACS getsources output. The outputs presented here are: the peak and integrated fluxes, the major and minor FWHM (noted A and B respectively) of the elliptic footprint and its position angle (PA). Tables C. 3 and C.4 give the Herschel-SPIRE and non-Herschel getsources outputs respectively. We note that the maps for all wavelengths used in the getsources extraction are also available at CDS.

\section{Appendix D: Multi-wavelength images and spectral energy distribution}

We present in this appendix the multi-wavelength images and spectral energy distributions (SEDs) for the 23 MDCs of NGC 6357, which are discussed in the main body of the text. 

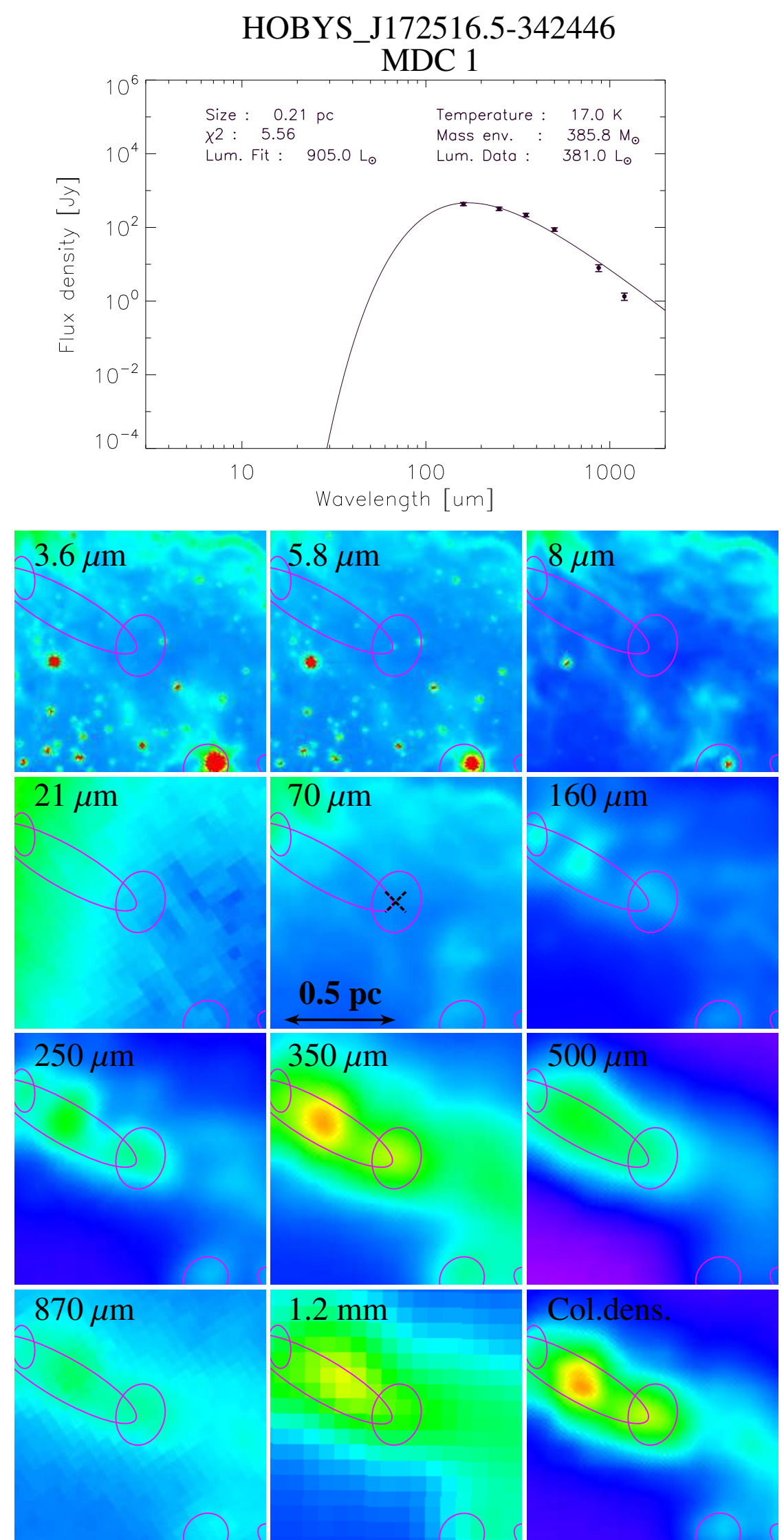

Fig. D.1. Upper panel: flux density versus wavelegnth (SED). The curve is the best fit model and the associated fitted values are indicated. Lower maps: $3.6,5.8,8,21 / 22 / 24,70,160,250,350,500$, and $870 \mu \mathrm{m}, 1.2 \mathrm{~mm}$, and high-resolution $\mathrm{N}_{H 2}$ column density maps. Ellipses represent the $160 \mu \mathrm{m}$ getsources footprints. The MDCs is identified by a cross on the $70 \mu \mathrm{m}$ image."Lum. Fit" is the flux integration under the fitted curve (noted $L_{\mathrm{Fit}}$ in the main text) while "Lum. Data." corresponds to the integral, using the trapezoid rule, over the finite number of data-points sampling the SED (noted $L_{\text {Data }}$ in the main text). 
D. Russeil et al.: Herschel-HOBYS study of the earliest phases of high-mass star formation in NGC 6357
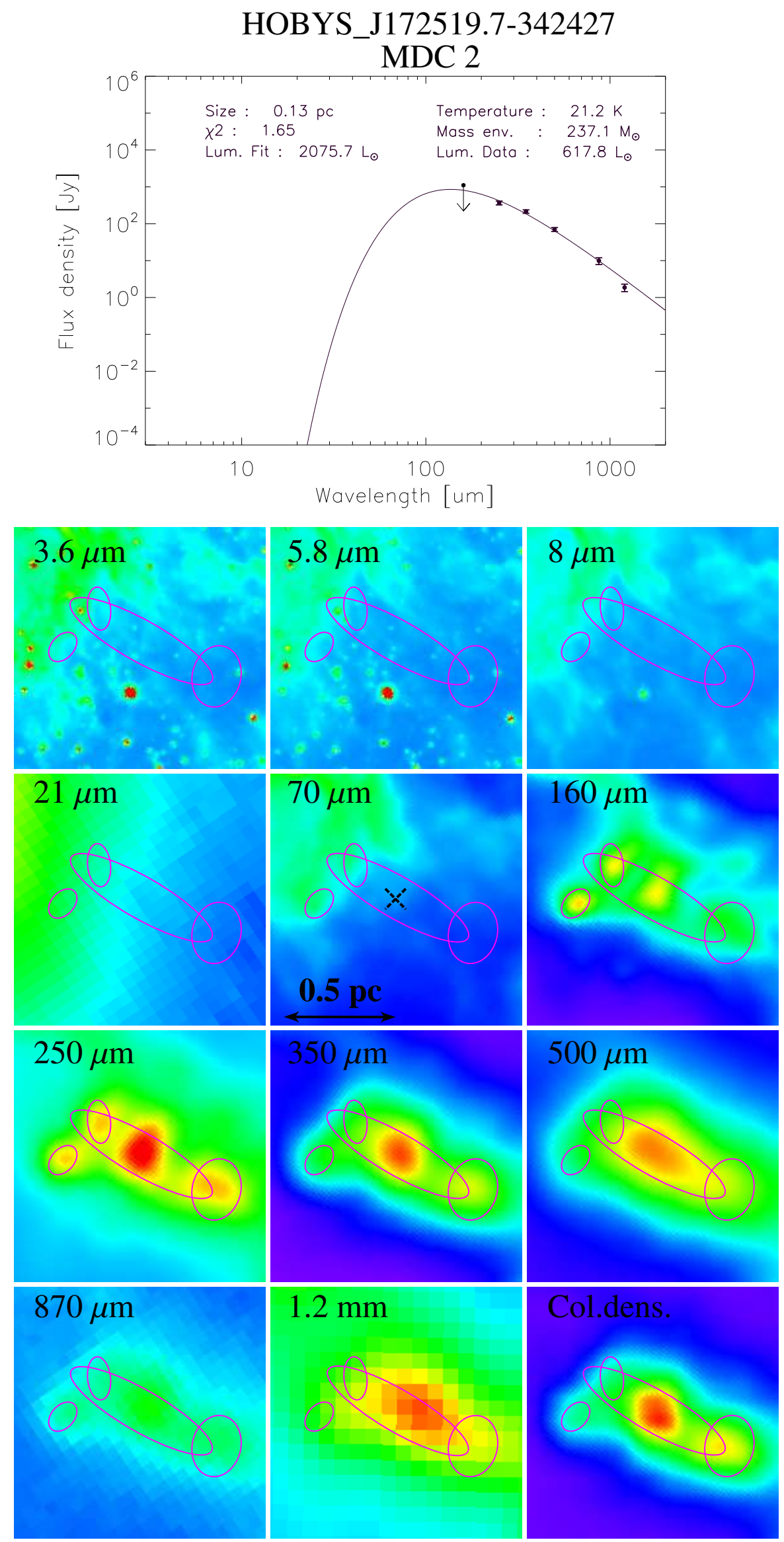

Fig. D.1. continued. 
A\&A 625, A134 (2019)
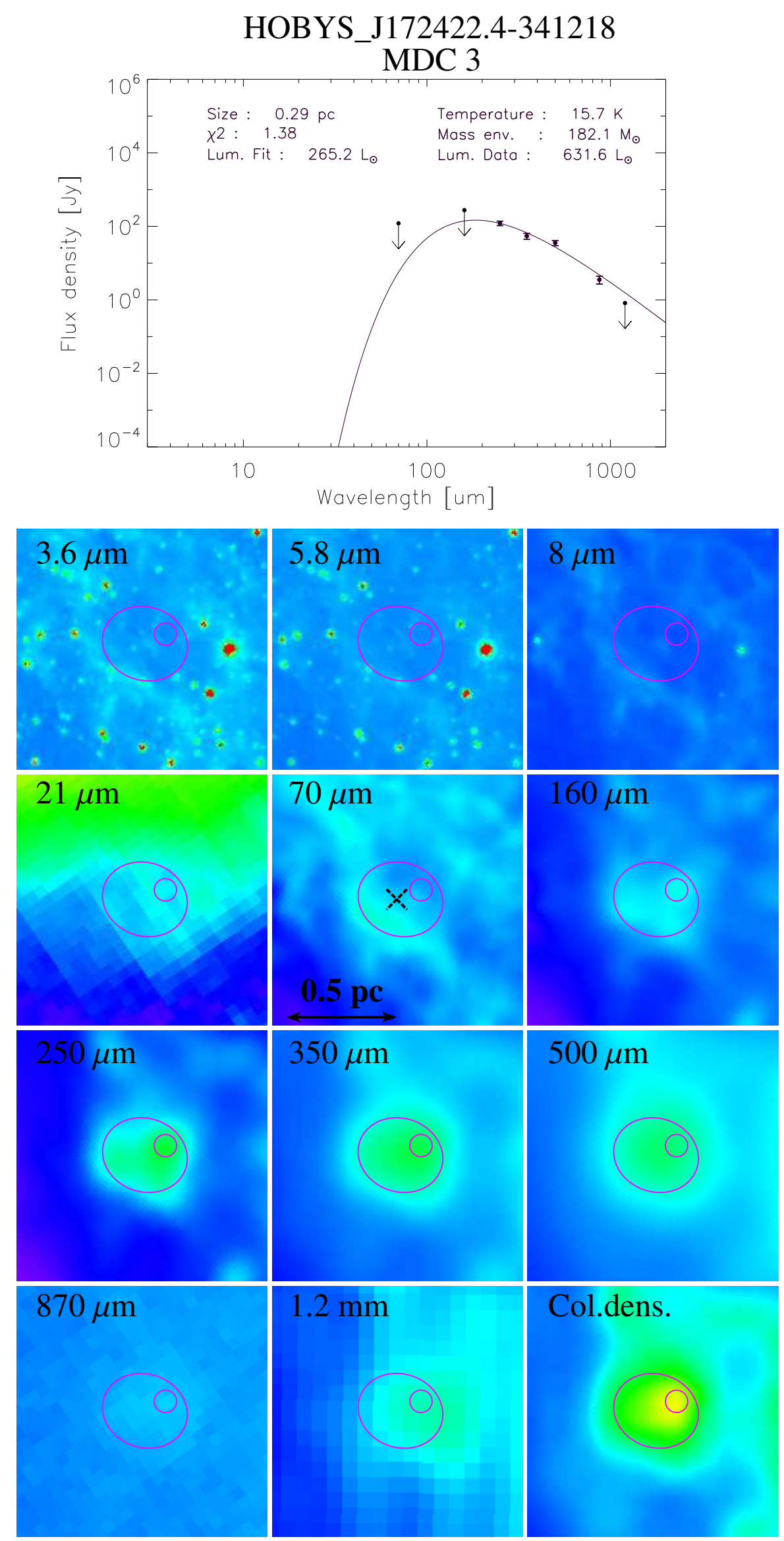

Fig. D.1. continued. 
D. Russeil et al.: Herschel-HOBYS study of the earliest phases of high-mass star formation in NGC 6357
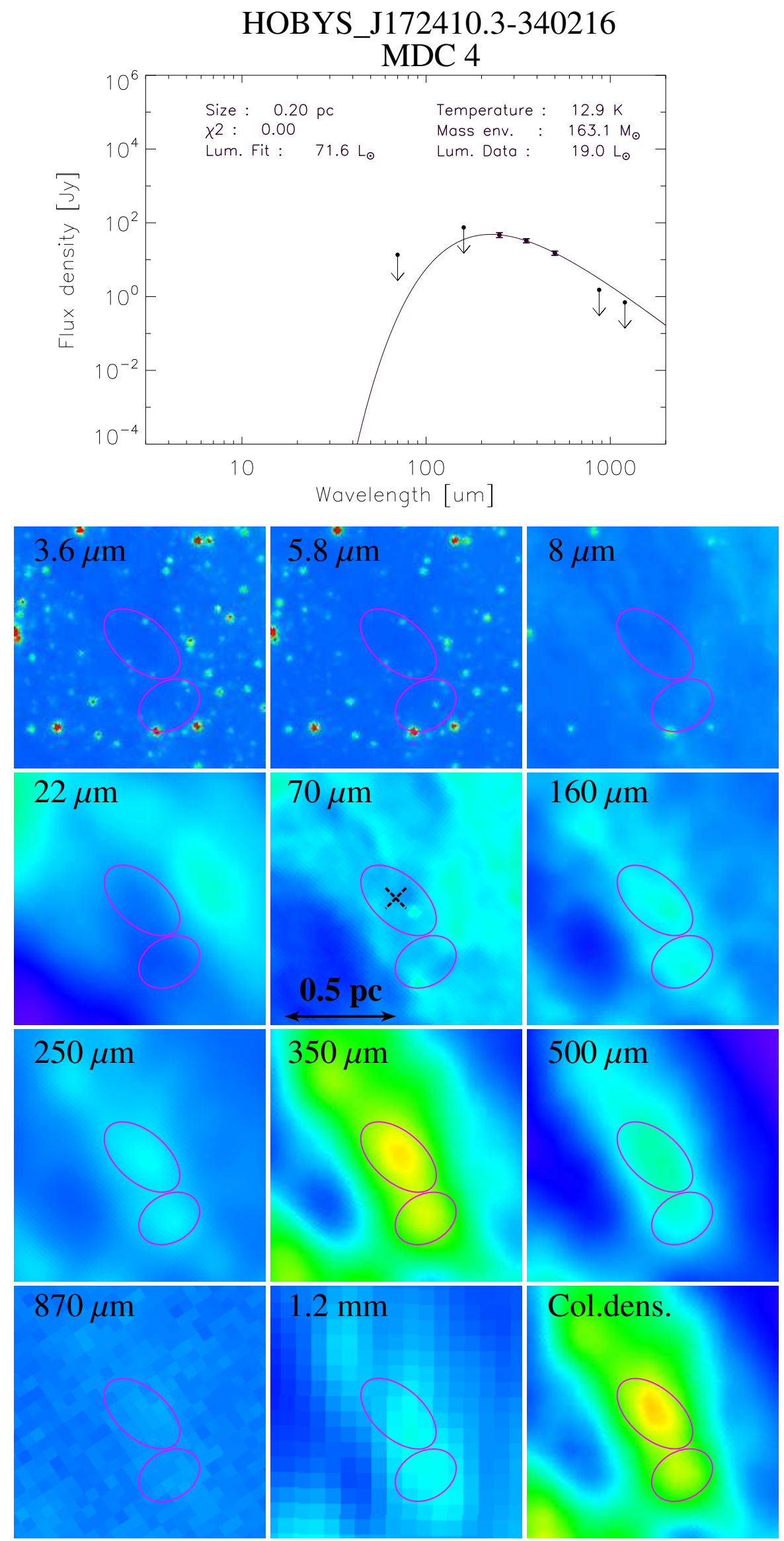

Fig. D.1. continued. 
A\&A 625, A134 (2019)
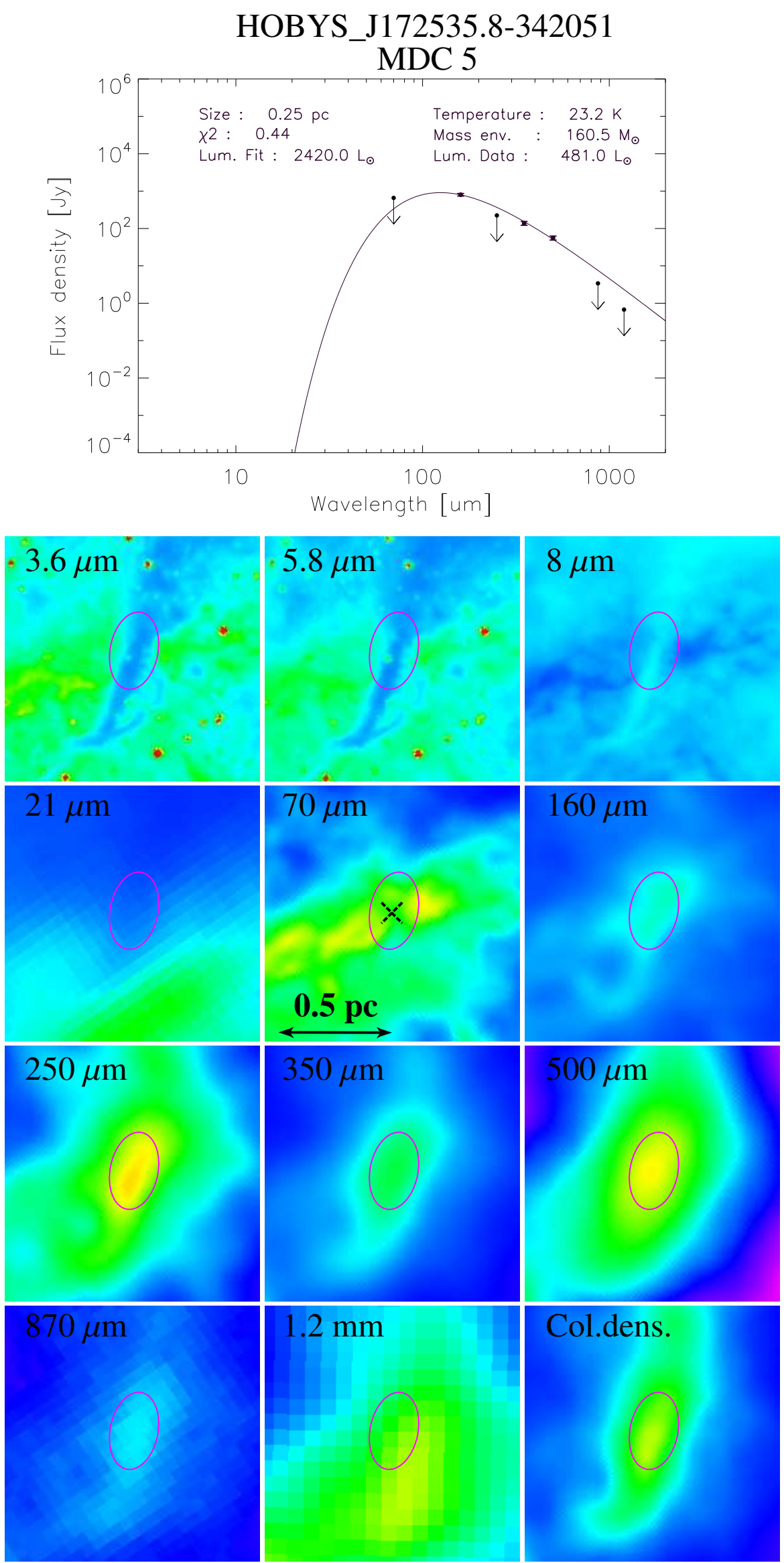

Fig. D.1. continued. 
D. Russeil et al.: Herschel-HOBYS study of the earliest phases of high-mass star formation in NGC 6357
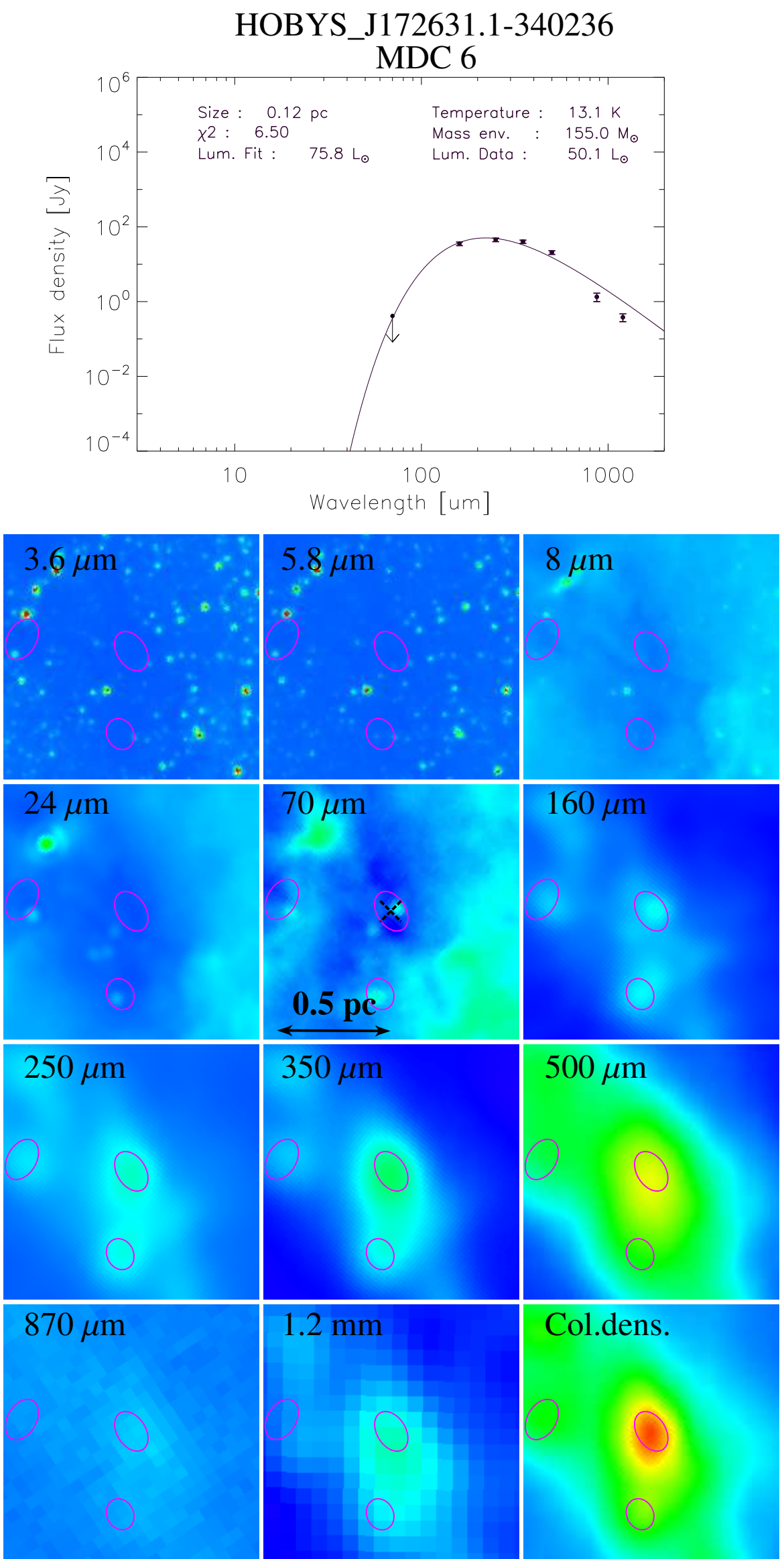

Fig. D.1. continued. 
A\&A 625, A134 (2019)
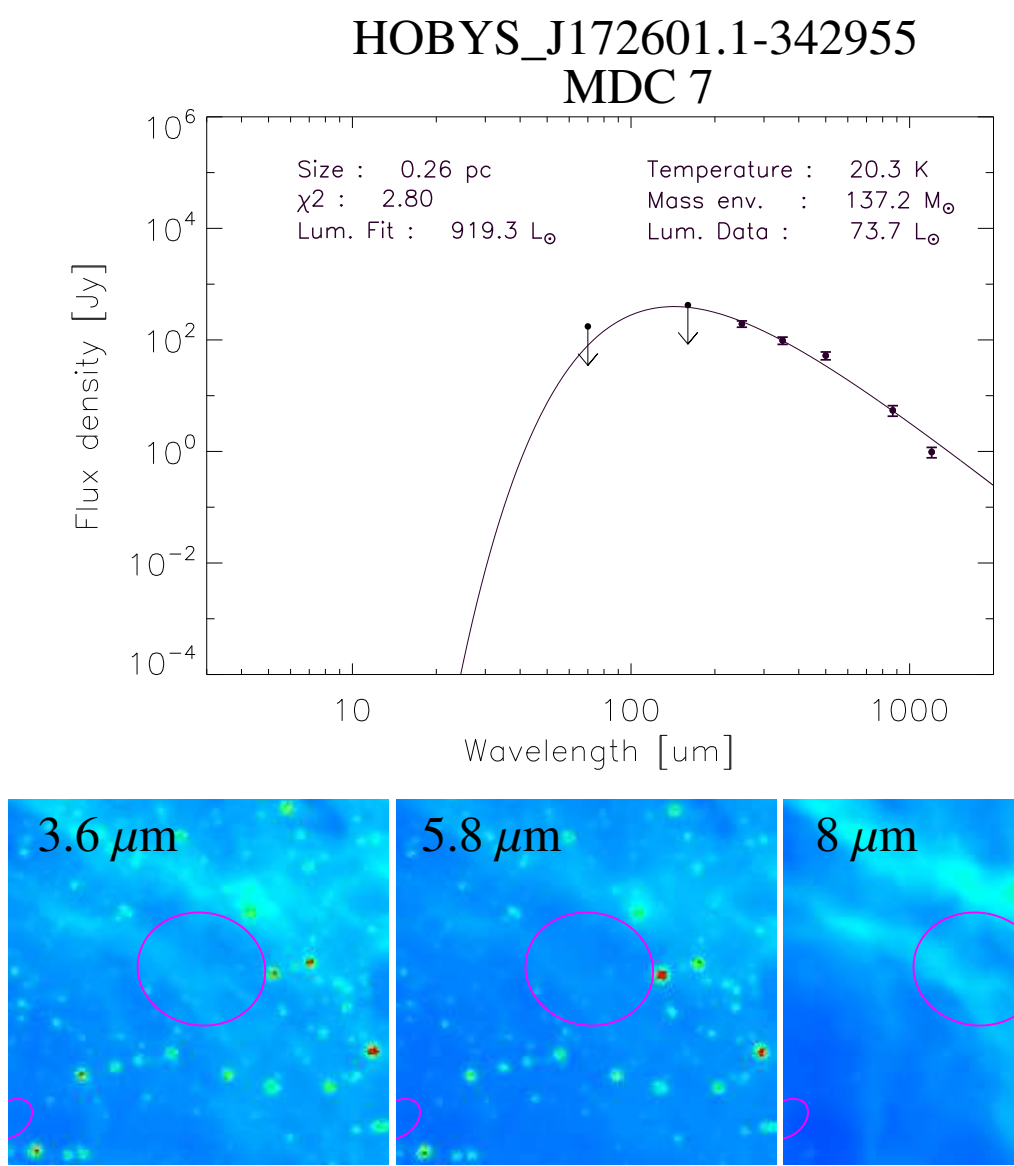

$8 \mu \mathrm{m}$

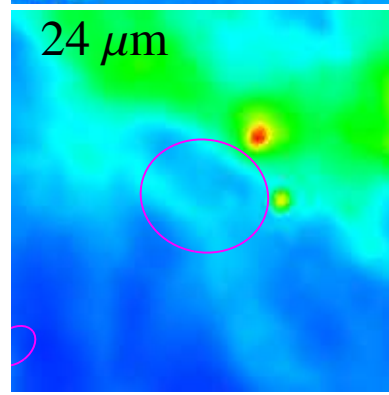

$70 \mu \mathrm{m}$

$160 \mu \mathrm{m}$
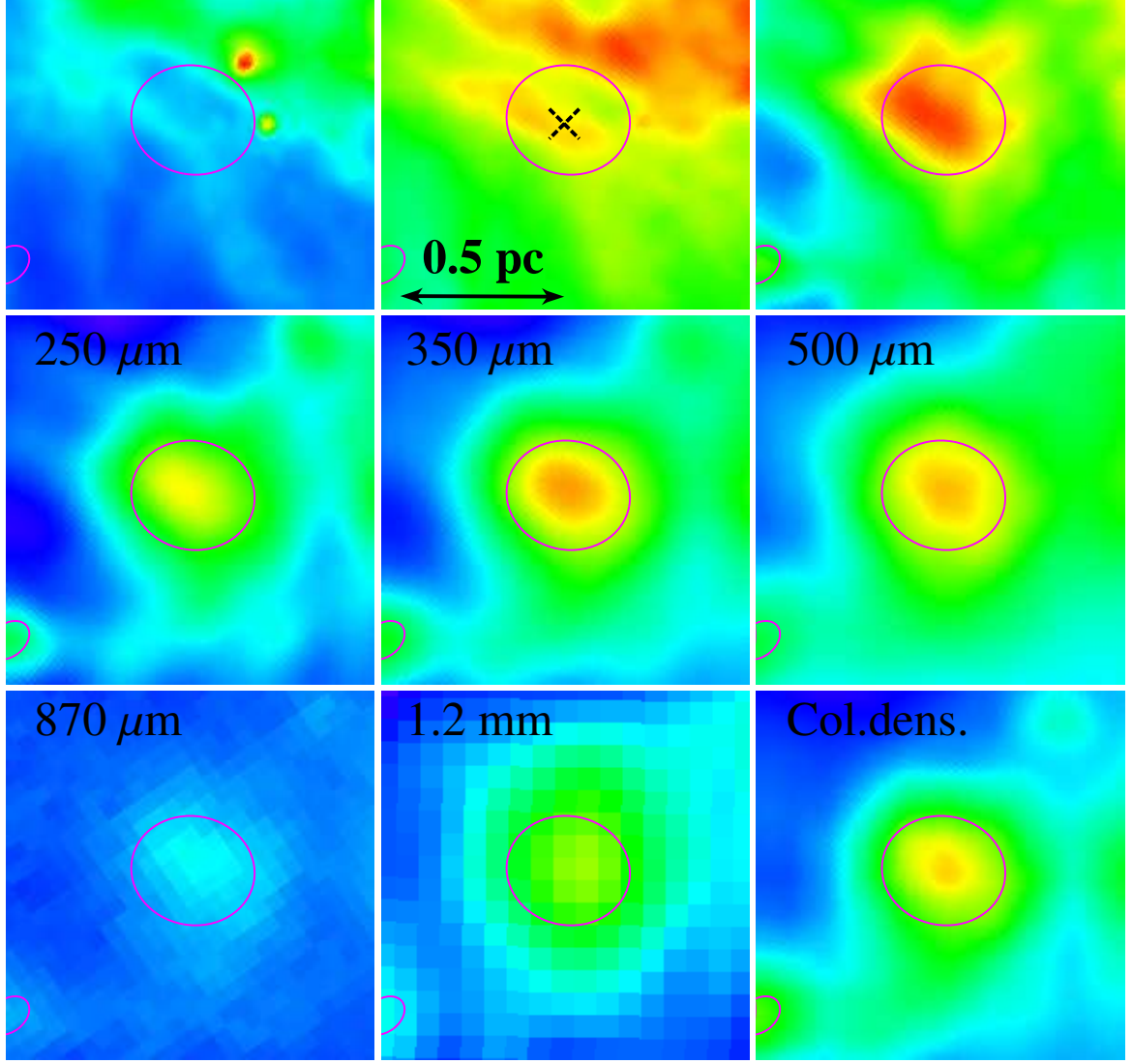

Fig. D.1. continued. 
D. Russeil et al.: Herschel-HOBYS study of the earliest phases of high-mass star formation in NGC 6357
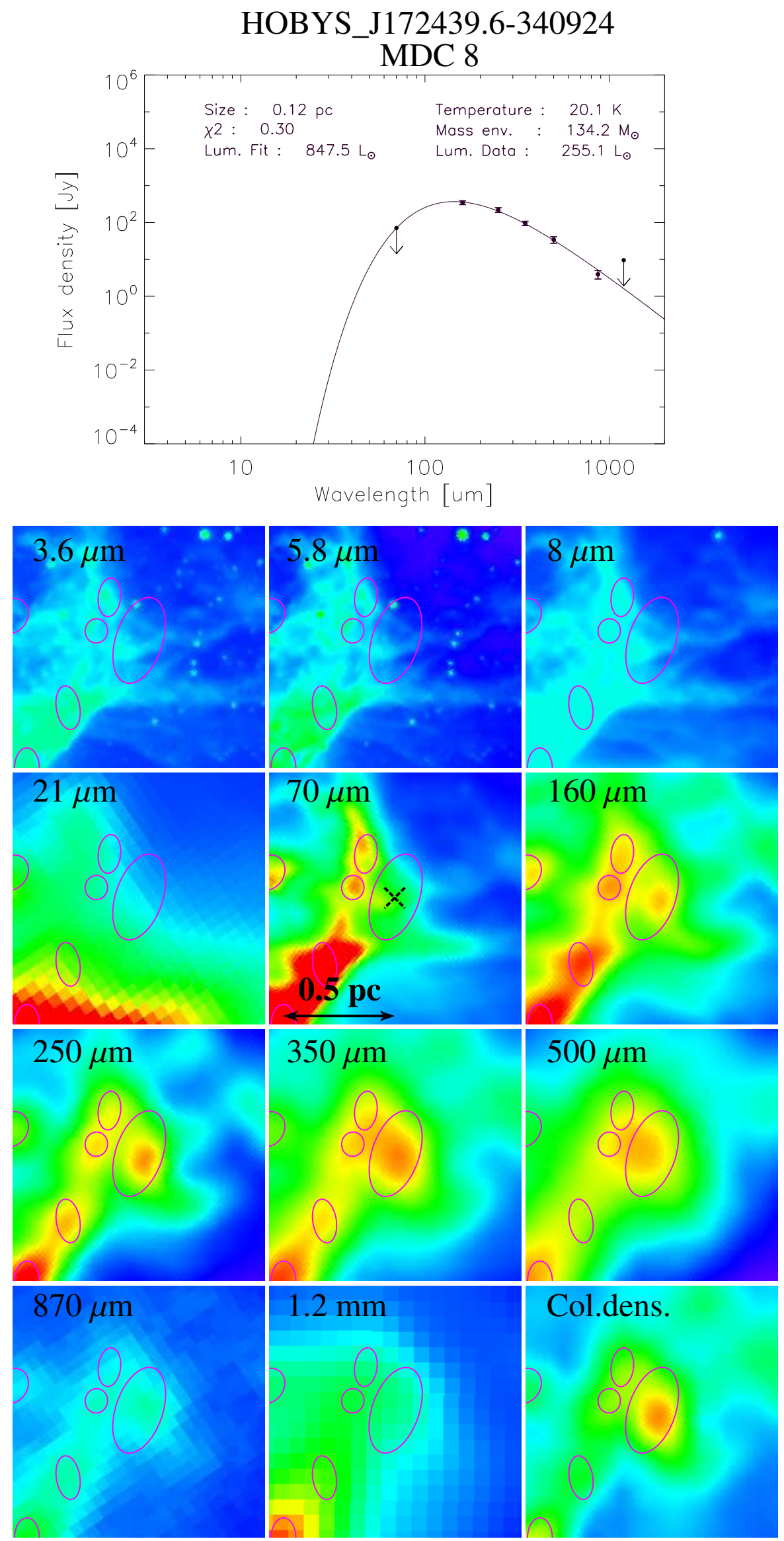

Fig. D.1. continued. 
A\&A 625, A134 (2019)
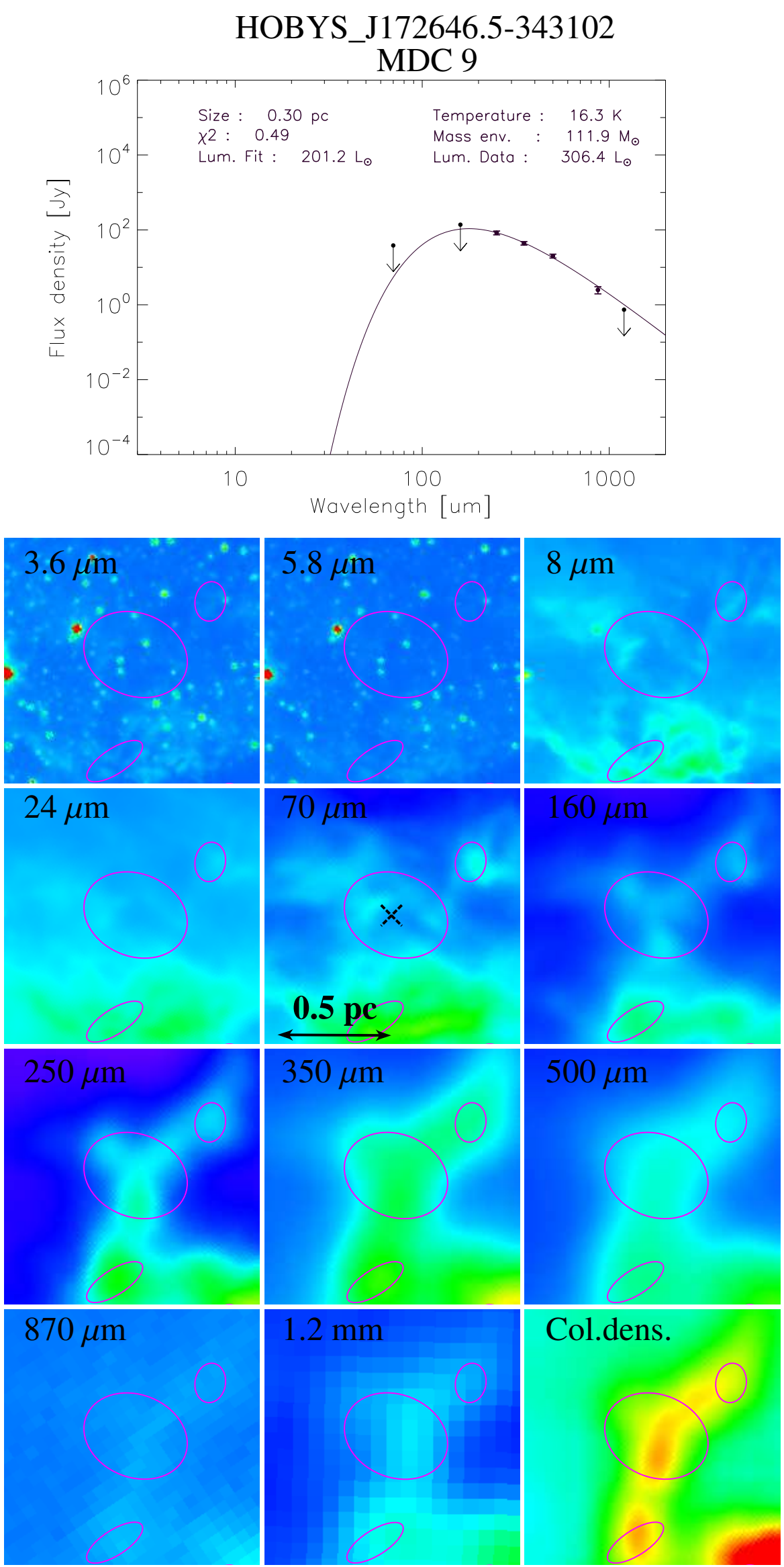

Fig. D.1. continued. 
D. Russeil et al.: Herschel-HOBYS study of the earliest phases of high-mass star formation in NGC 6357
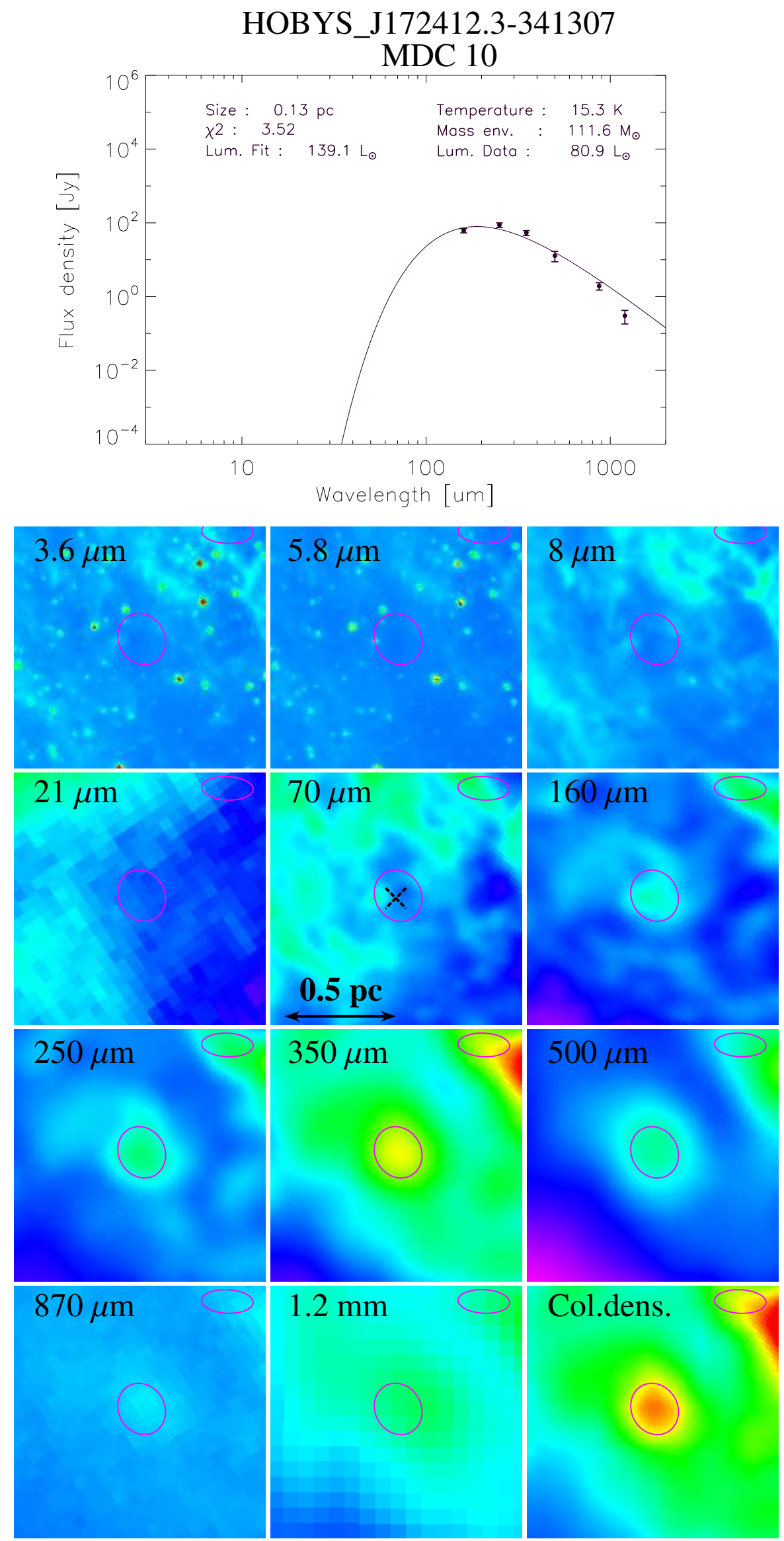

Fig. D.1. continued. 
A\&A 625, A134 (2019)
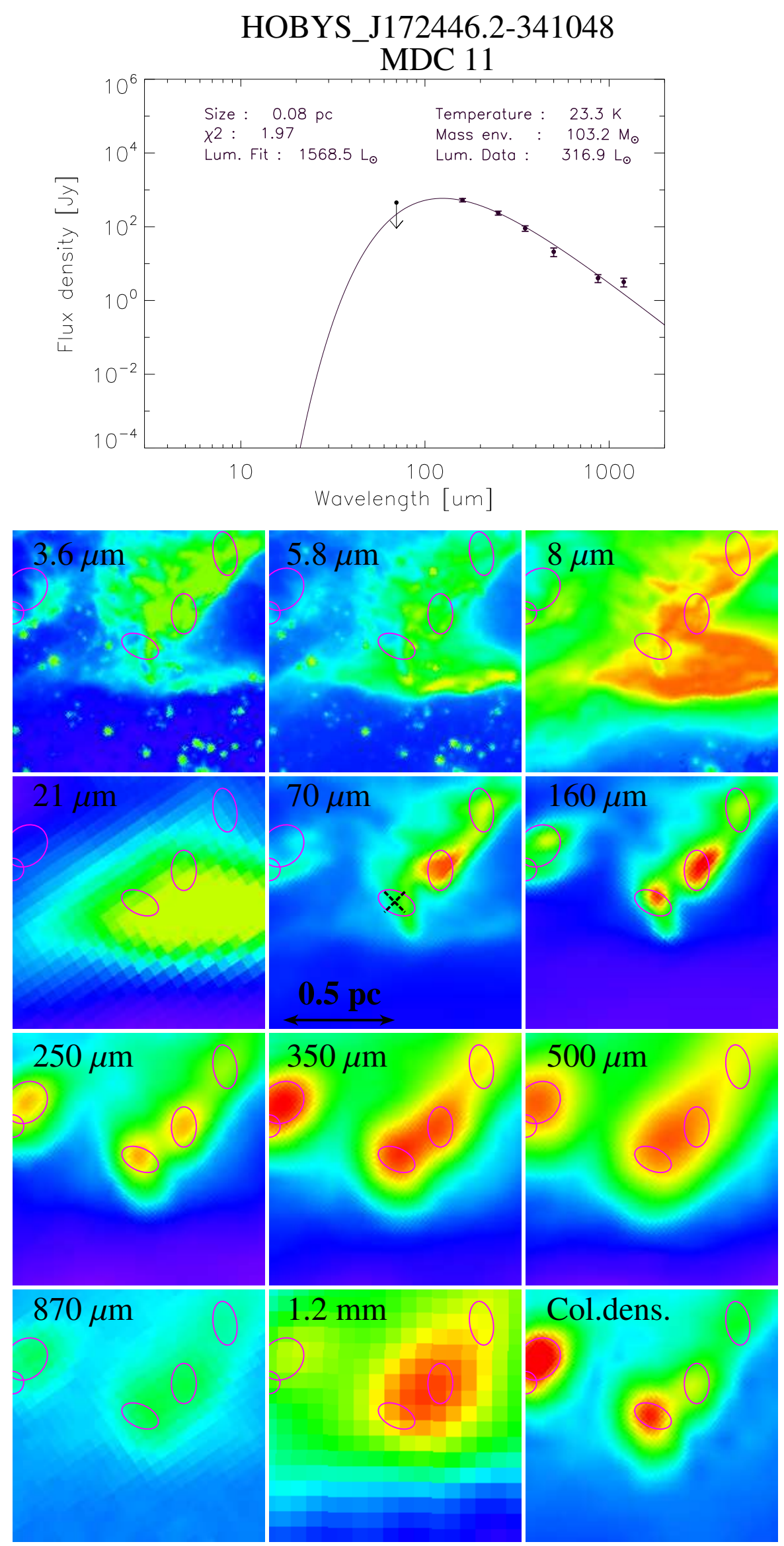

Fig. D.1. continued. 
D. Russeil et al.: Herschel-HOBYS study of the earliest phases of high-mass star formation in NGC 6357
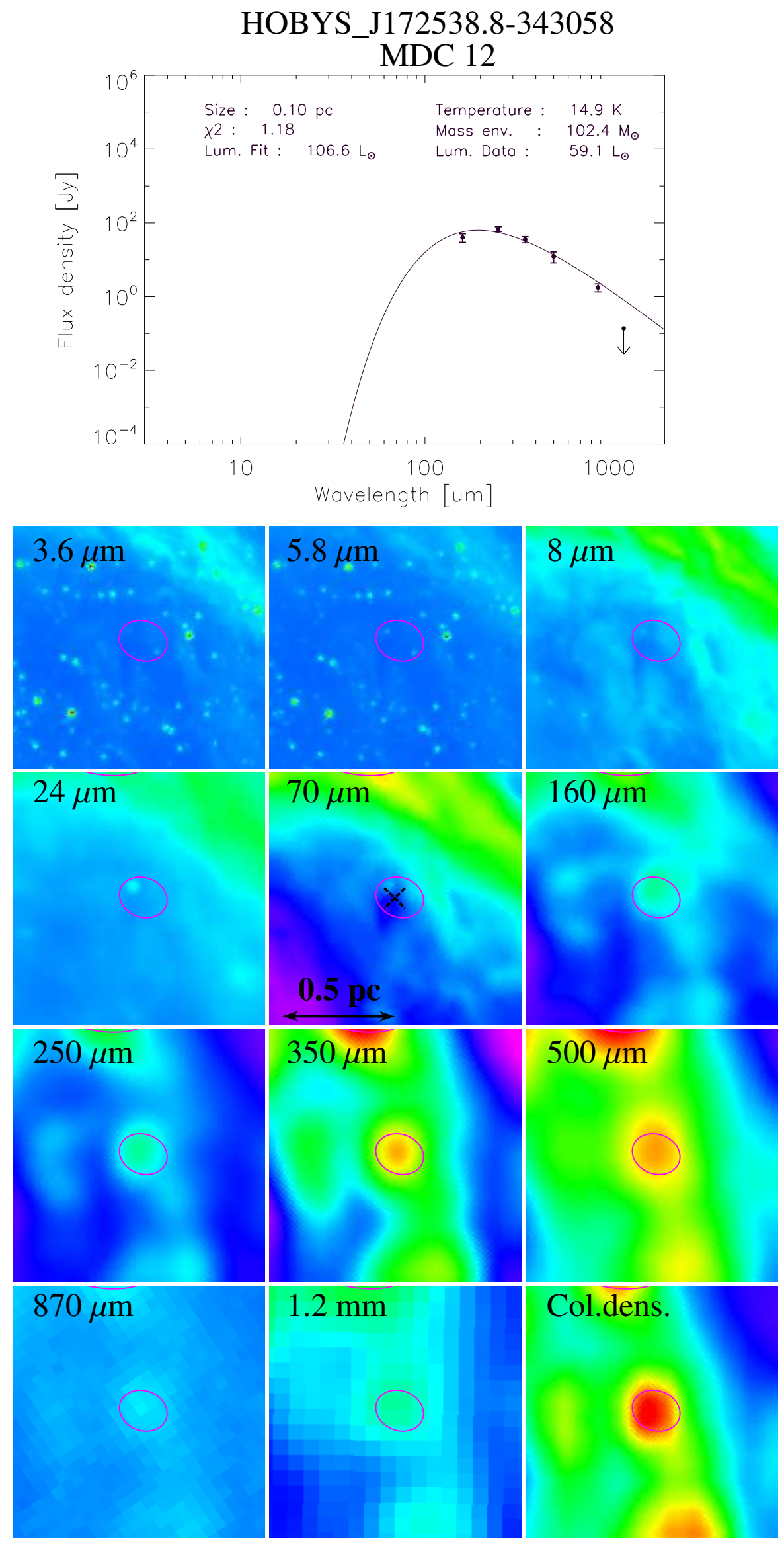

Fig. D.1. continued. 
A\&A 625, A134 (2019)
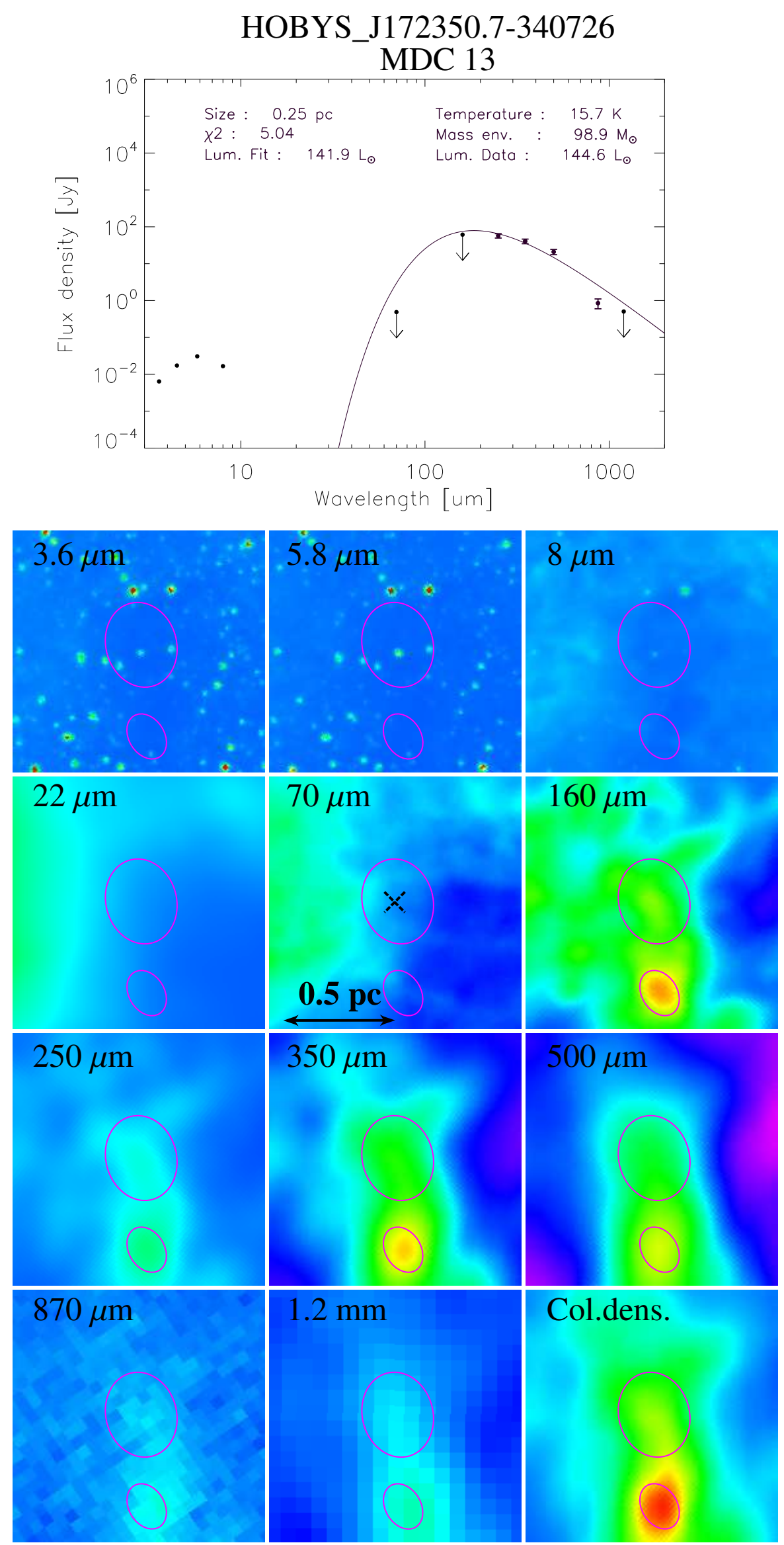

Fig. D.1. continued. 
D. Russeil et al.: Herschel-HOBYS study of the earliest phases of high-mass star formation in NGC 6357
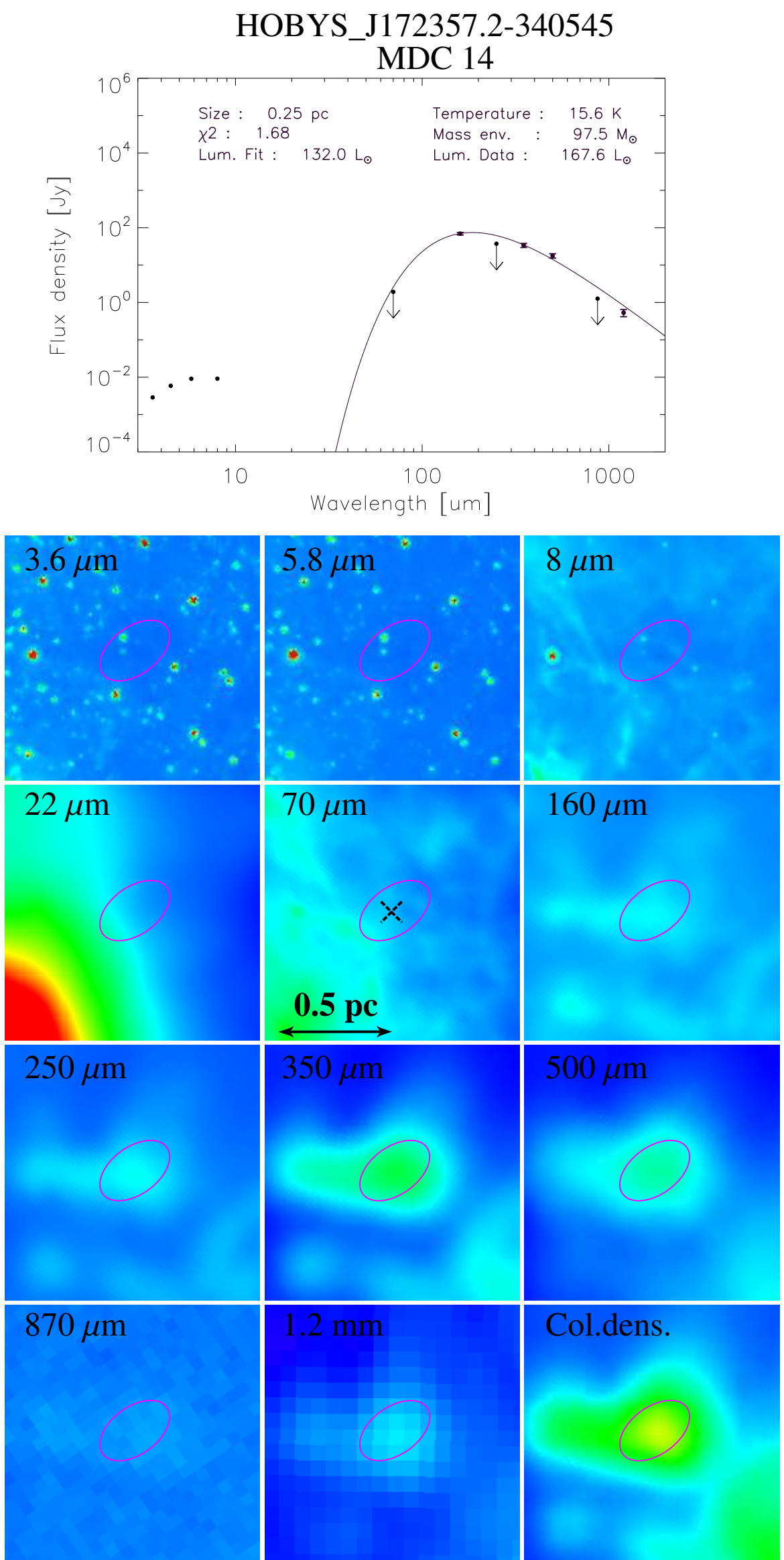

Fig. D.1. continued. 
A\&A 625, A134 (2019)
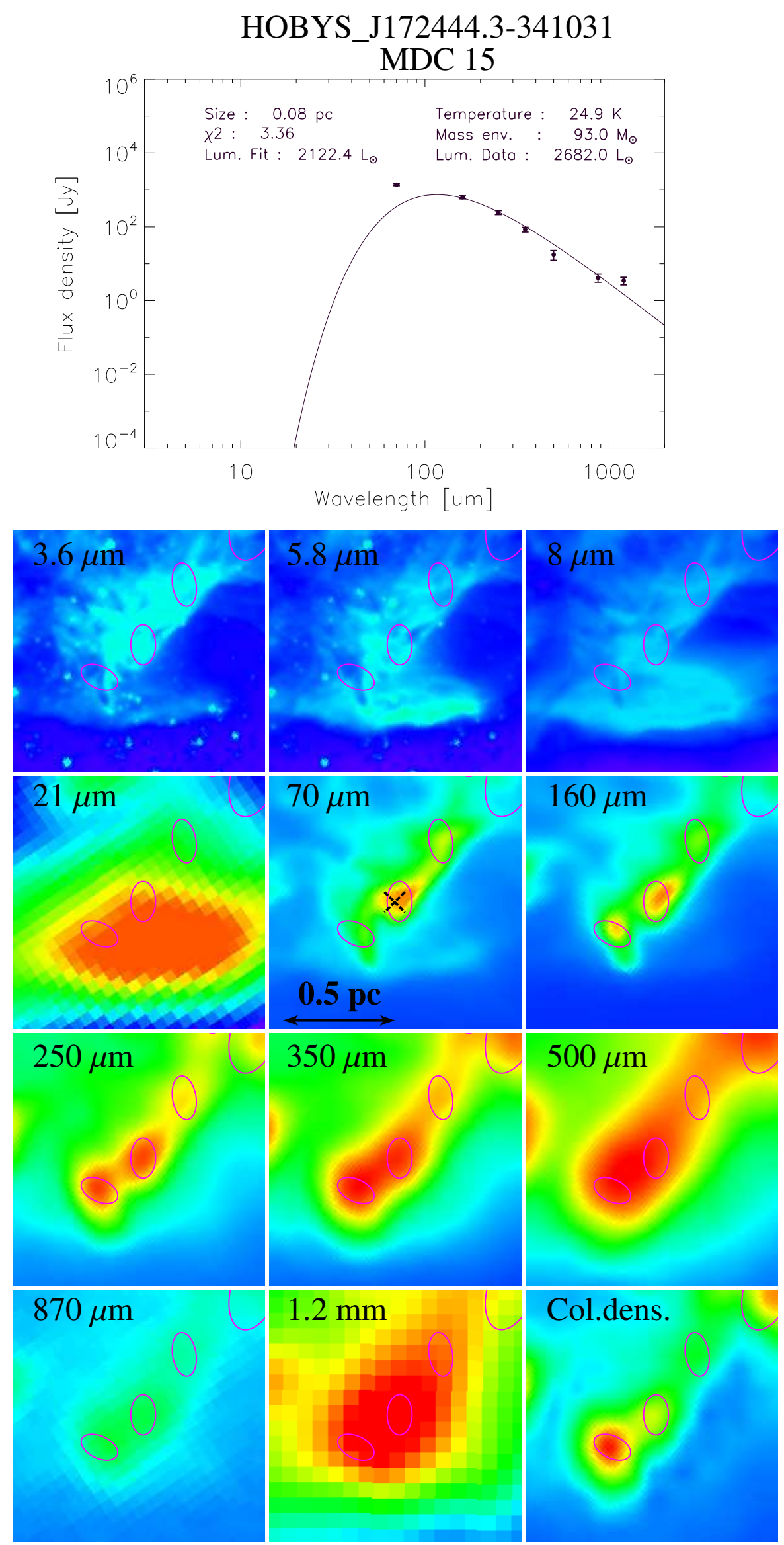

Fig. D.1. continued. 
D. Russeil et al.: Herschel-HOBYS study of the earliest phases of high-mass star formation in NGC 6357
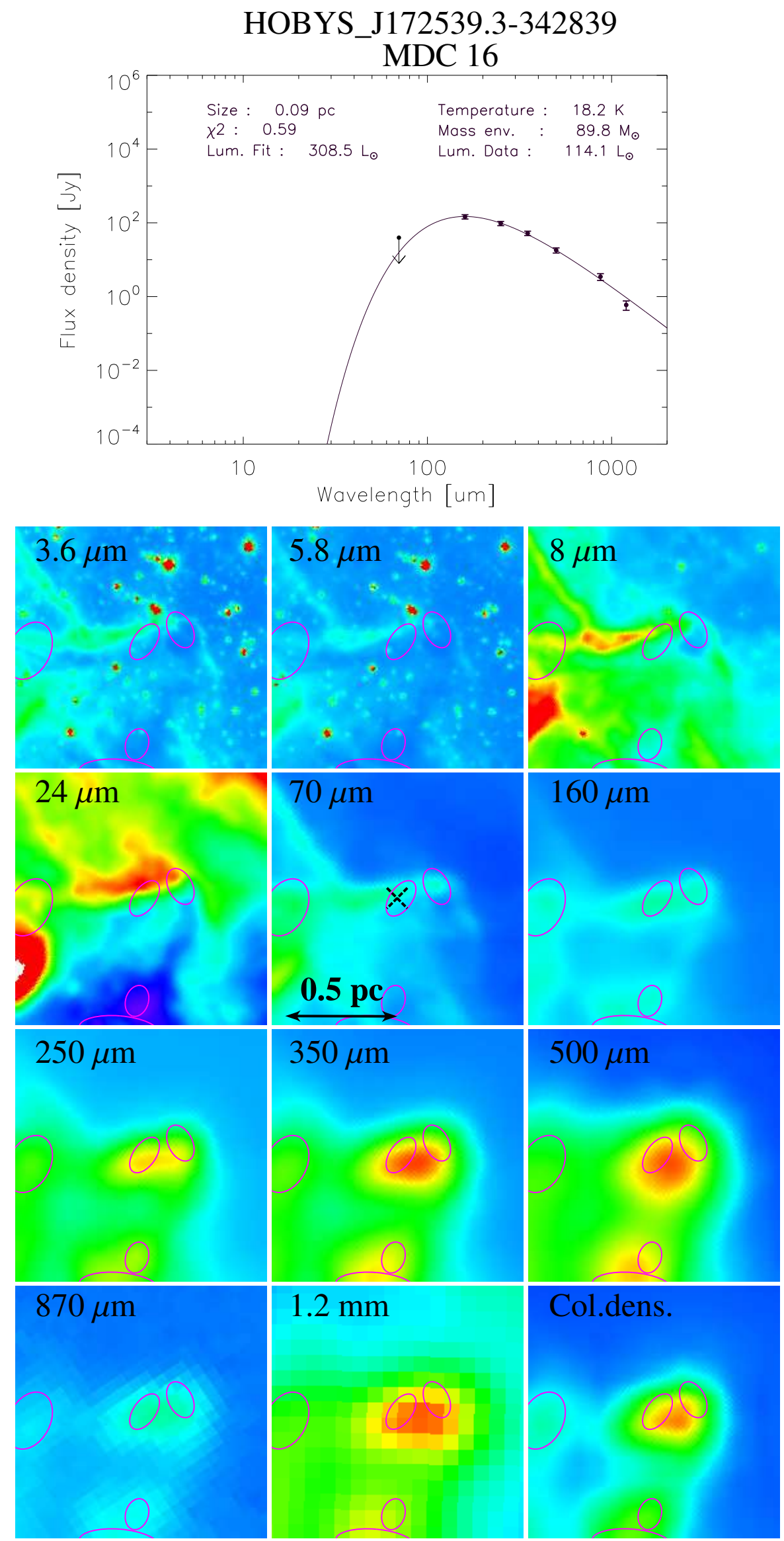

Fig. D.1. continued. 
A\&A 625, A134 (2019)
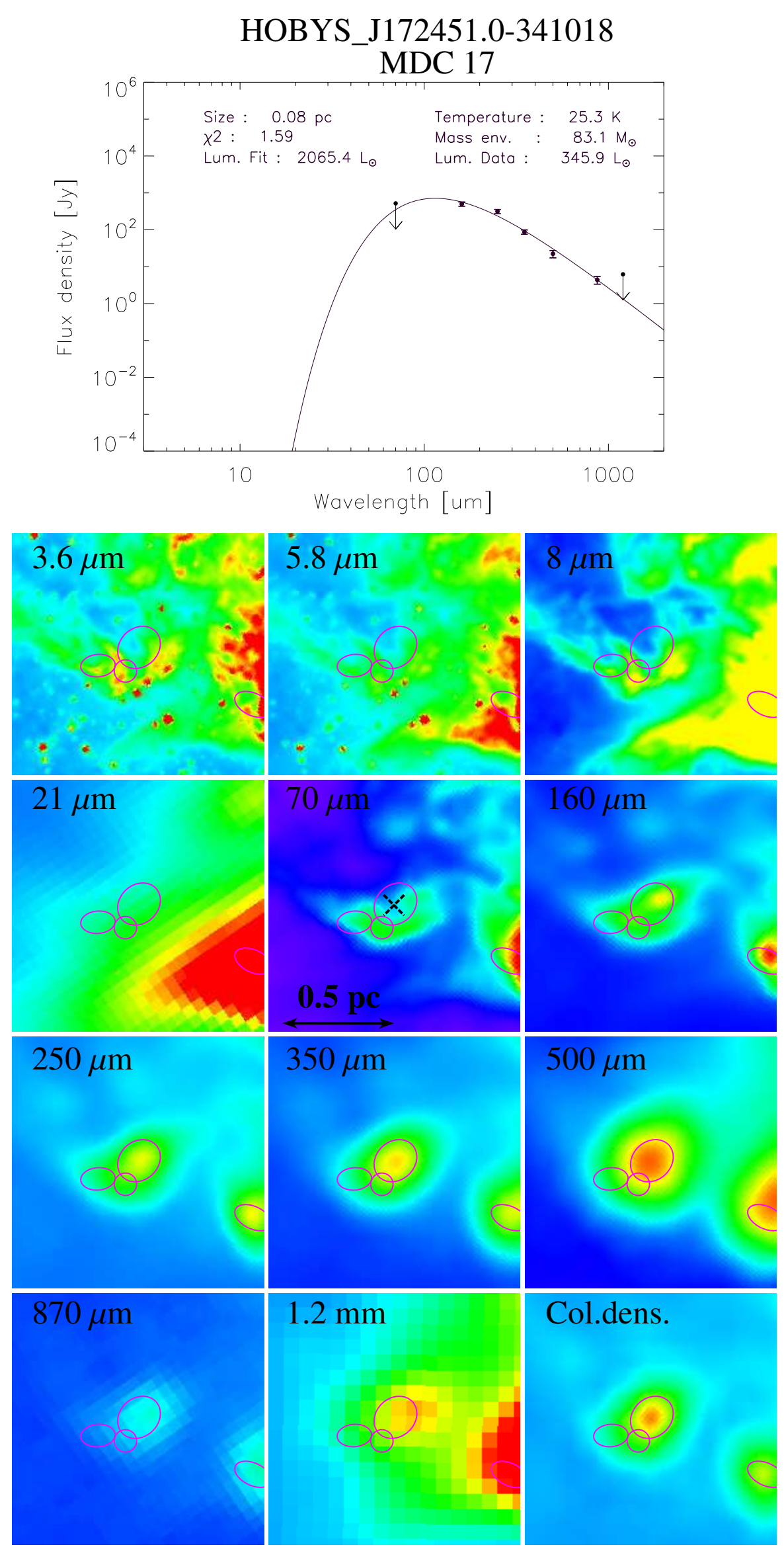

Fig. D.1. continued. 
D. Russeil et al.: Herschel-HOBYS study of the earliest phases of high-mass star formation in NGC 6357
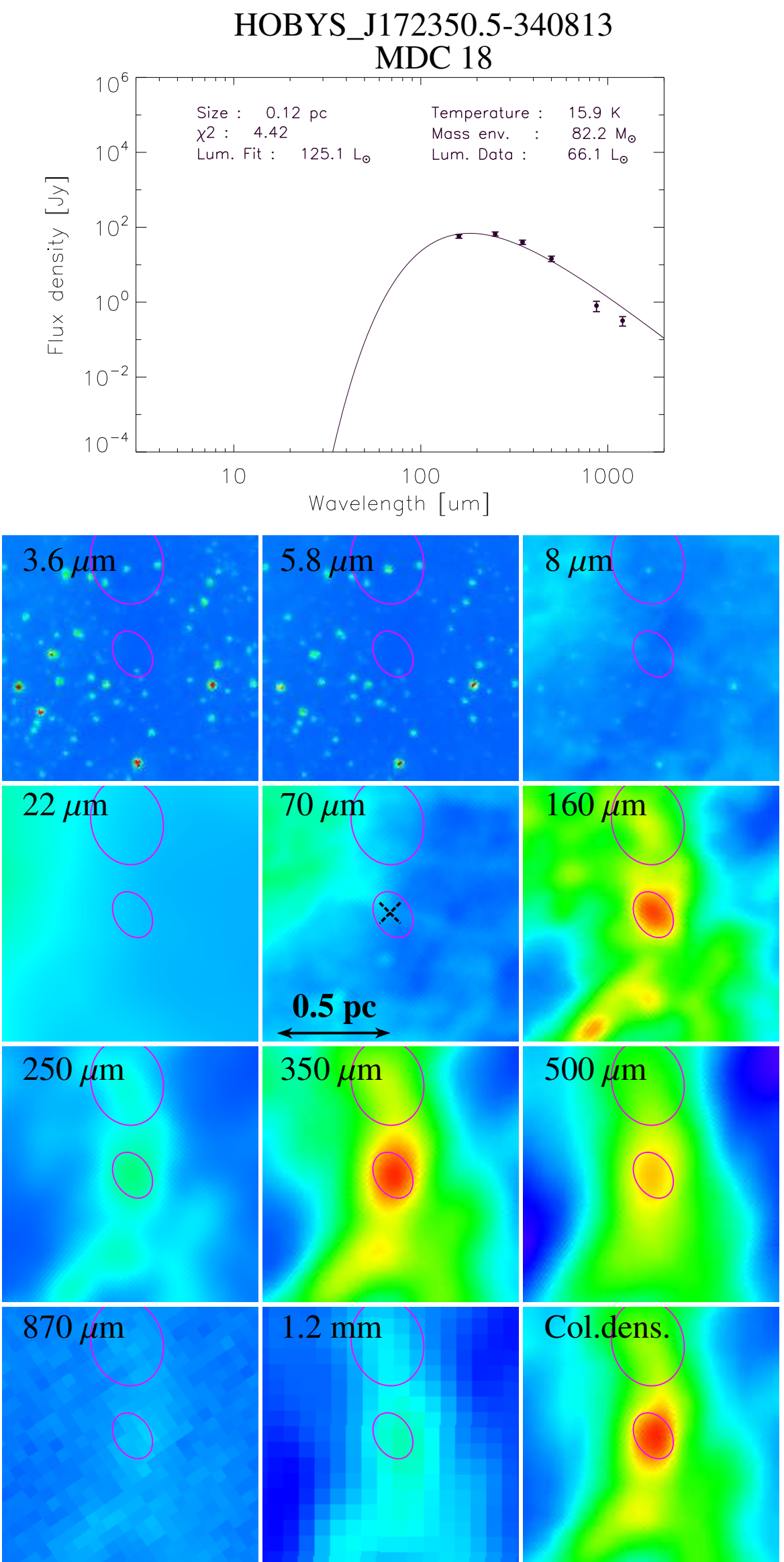

Fig. D.1. continued. 
A\&A 625, A134 (2019)
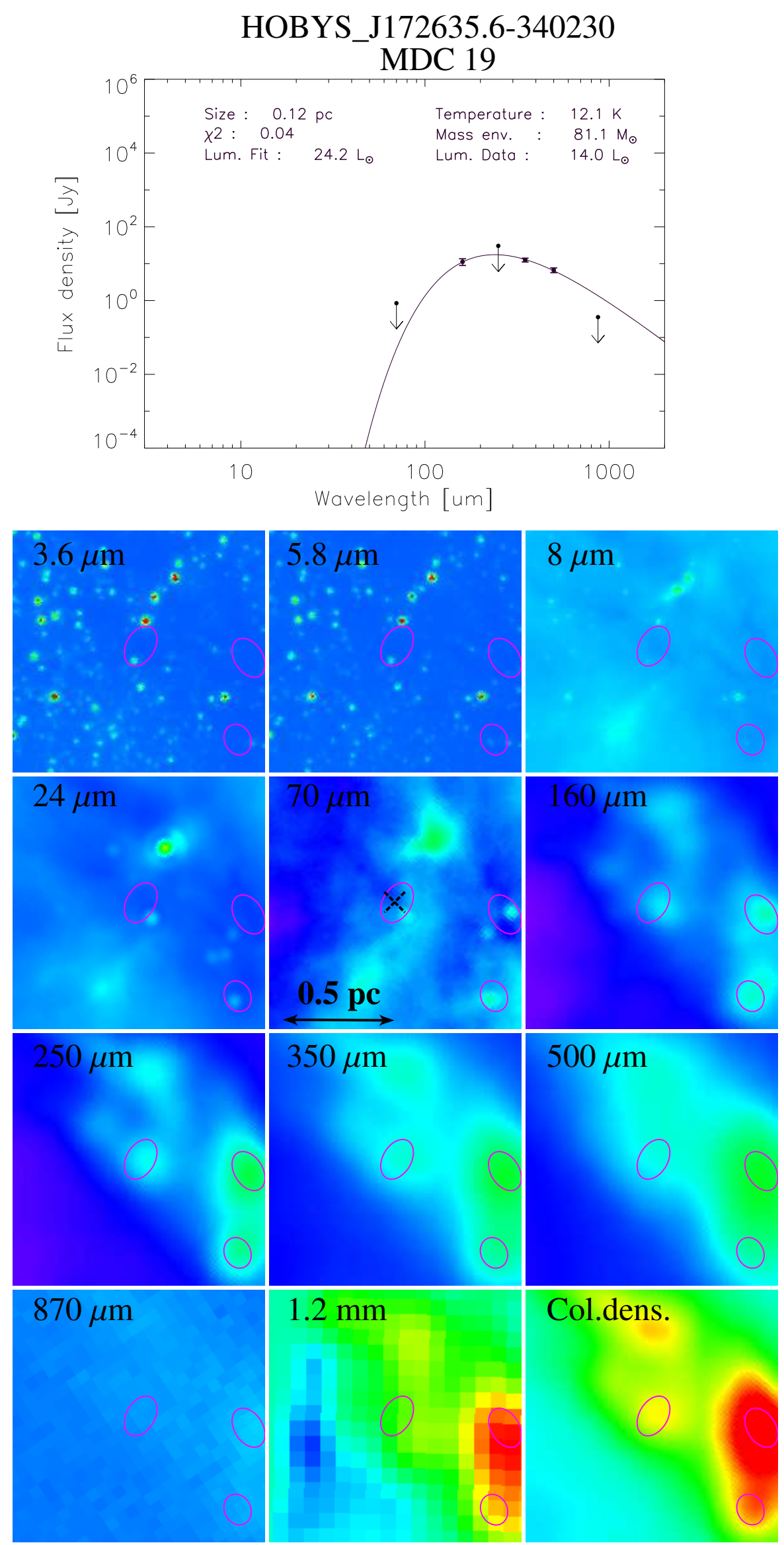

Fig. D.1. continued. 
D. Russeil et al.: Herschel-HOBYS study of the earliest phases of high-mass star formation in NGC 6357
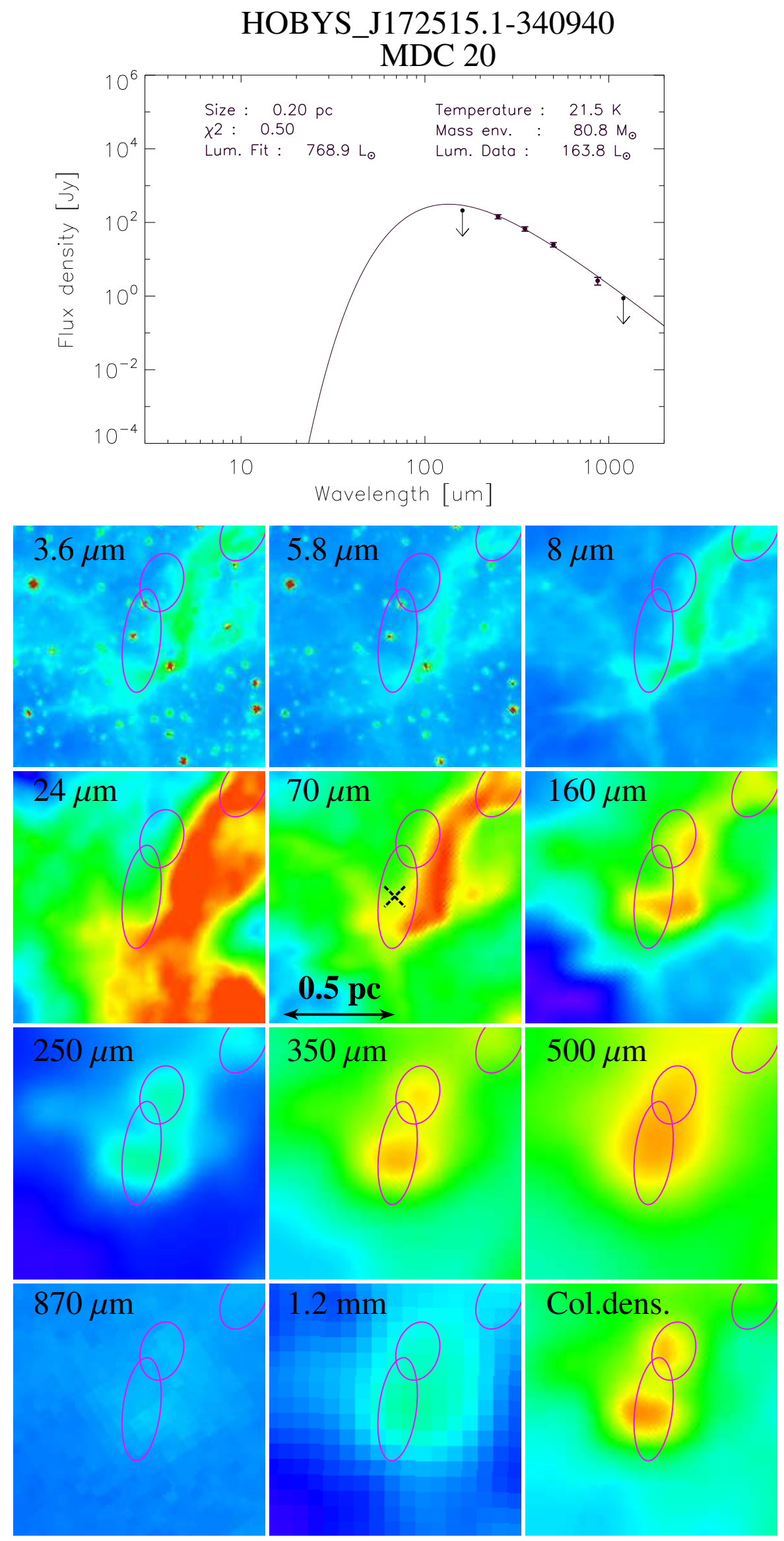

Fig. D.1. continued. 
A\&A 625, A134 (2019)
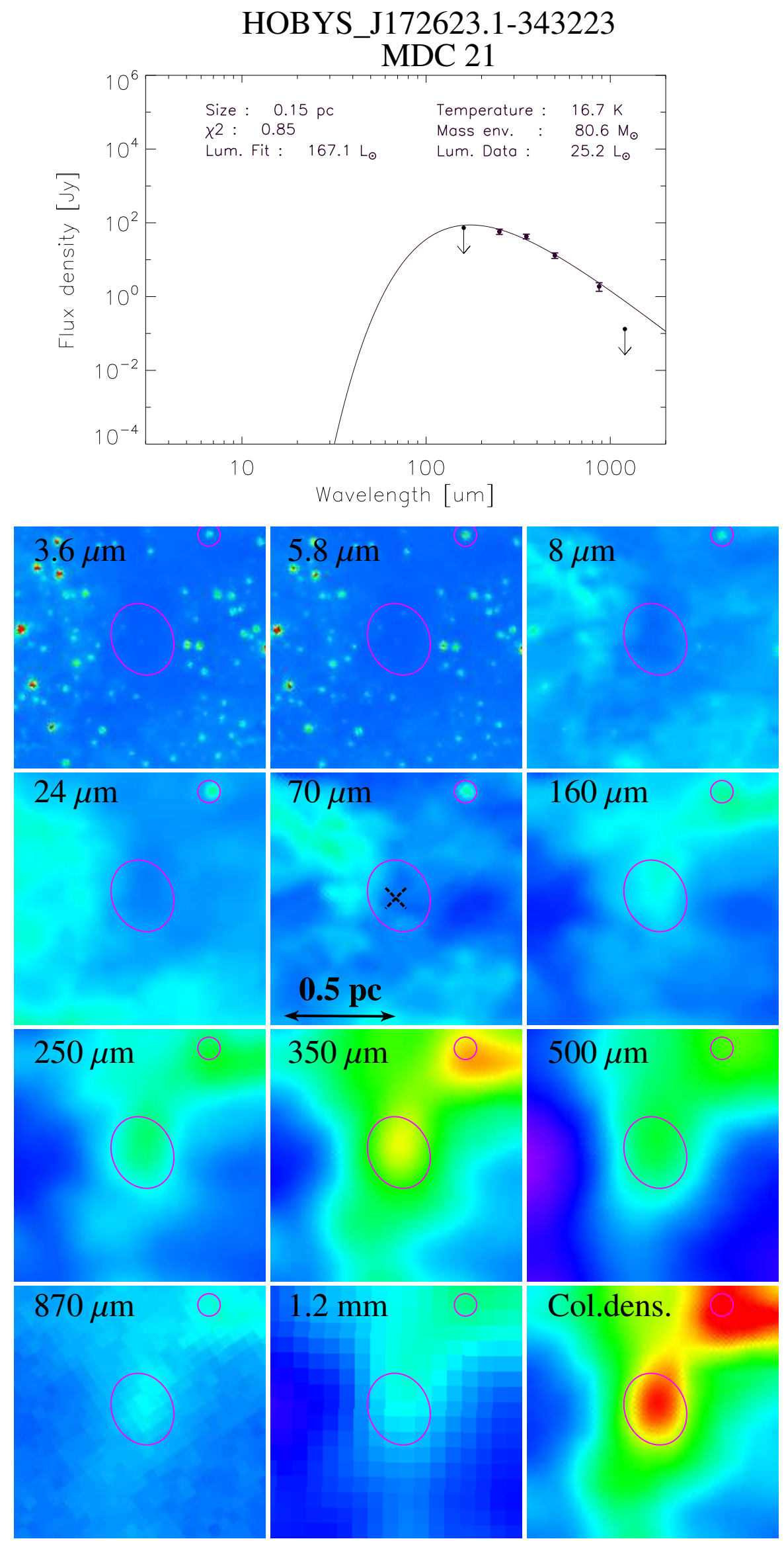

Fig. D.1. continued. 
D. Russeil et al.: Herschel-HOBYS study of the earliest phases of high-mass star formation in NGC 6357
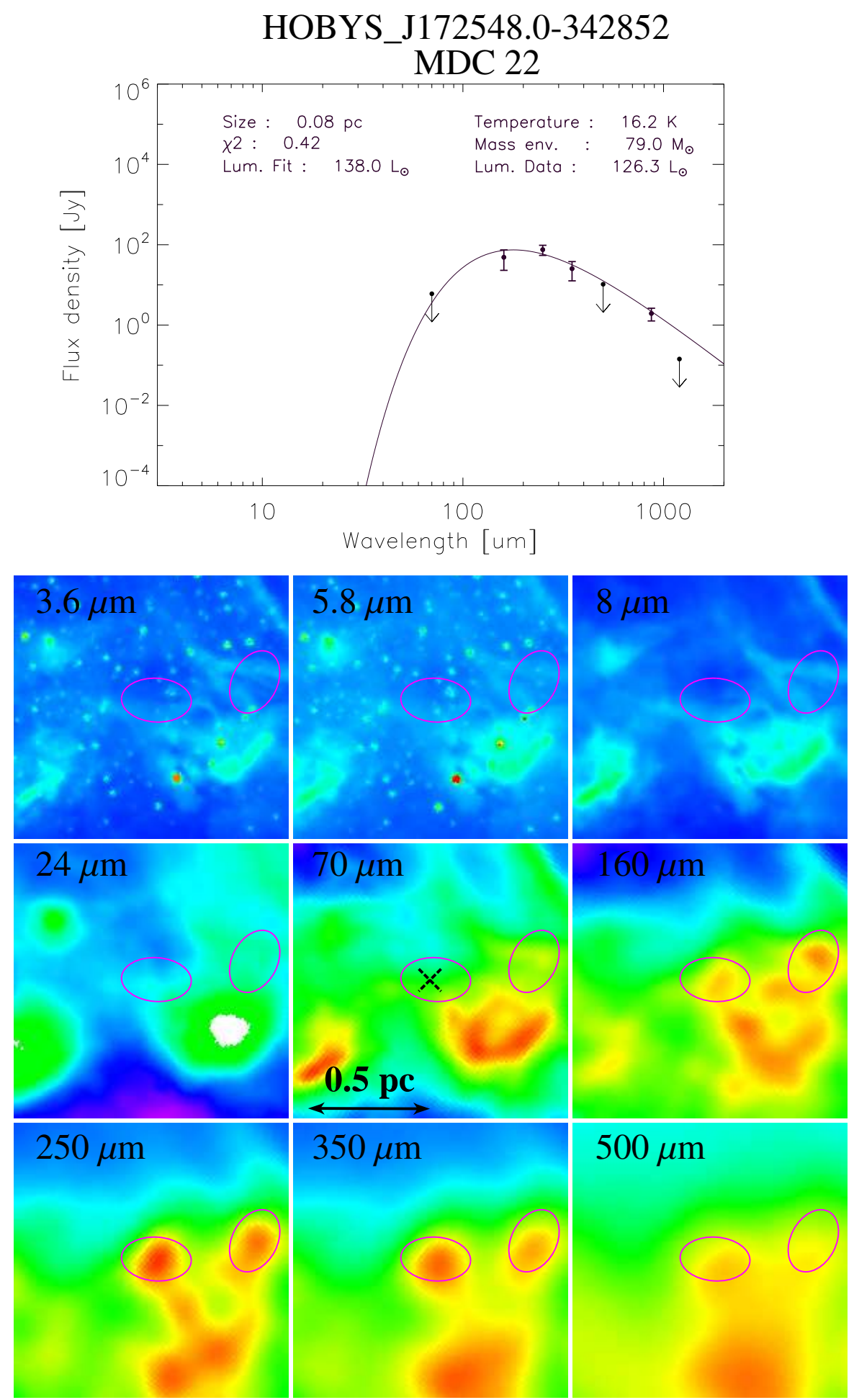

$350 \mu \mathrm{m}$

$500 \mu \mathrm{m}$
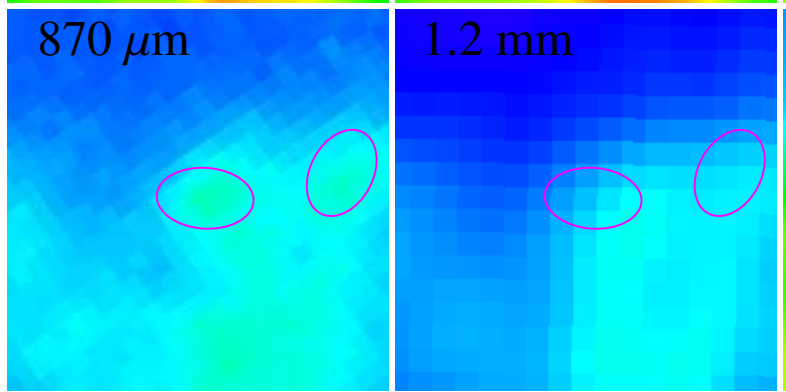

\section{Col.dens.}

Fig. D.1. continued. 
A\&A 625, A134 (2019)
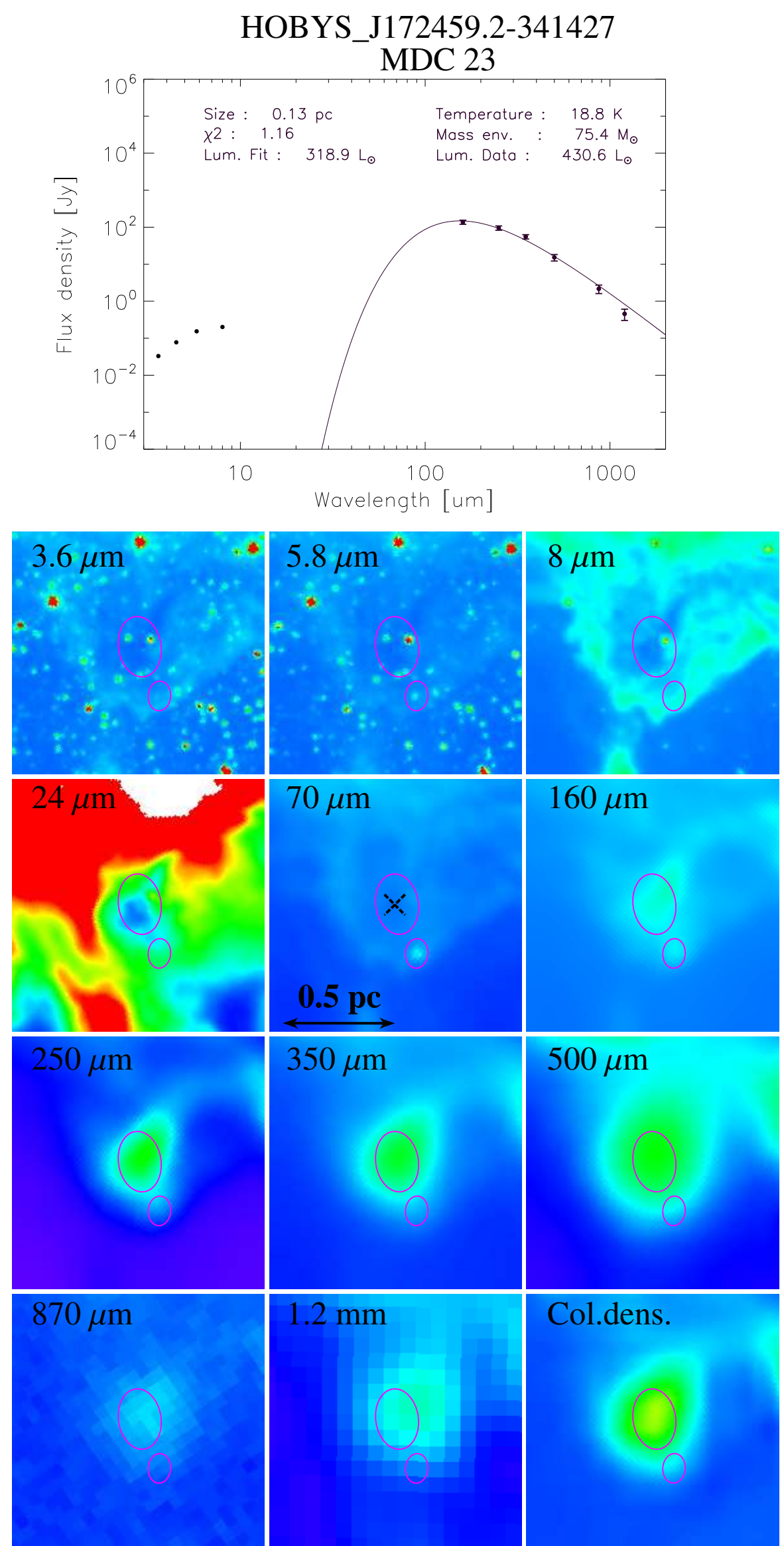

Fig. D.1. continued. 